\title{
Evaluation of non-invasive biomarkers for carcinogenic exposure to cigarette smoke
}

\author{
Girish Srinivas Gudi \\ West Virginia University
}

Follow this and additional works at: https://researchrepository.wvu.edu/etd

\section{Recommended Citation}

Gudi, Girish Srinivas, "Evaluation of non-invasive biomarkers for carcinogenic exposure to cigarette smoke" (1999). Graduate Theses, Dissertations, and Problem Reports. 97.

https://researchrepository.wvu.edu/etd/97

This Thesis is protected by copyright and/or related rights. It has been brought to you by the The Research Repository @ WVU with permission from the rights-holder(s). You are free to use this Thesis in any way that is permitted by the copyright and related rights legislation that applies to your use. For other uses you must obtain permission from the rights-holder(s) directly, unless additional rights are indicated by a Creative Commons license in the record and/ or on the work itself. This Thesis has been accepted for inclusion in WVU Graduate Theses, Dissertations, and Problem Reports collection by an authorized administrator of The Research Repository @ WVU. For more information, please contact researchrepository@mail.wvu.edu. 


\title{
EVALUATION OF NON-INVASIVE BIOMARKERS FOR CARCINOGENIC EXPOSURE TO CIGARETTE SMOKE
}

\author{
Girish Srinivas Gudi
}

\author{
A THESIS \\ Submitted to \\ The School of Pharmacy \\ at \\ West Virginia University \\ in partial fulfillment of the requirements \\ for the degree of \\ MASTER OF SCIENCE \\ in \\ Pharmaceutical Sciences \\ Mohamadi Sarkar, Ph.D., Chair \\ Ainsley Weston, Ph.D. \\ Timothy Tracy, Ph.D \\ Ann Berry, Ph.D \\ Department of Basic Pharmaceutical Sciences \\ Morgantown, West Virginia
}

1999

Keywords: benzopyrene, pyrene, tetrol, pyrenol, 1-hydroxypyrene glucuronide, urinary, urine, metabolites, carcinogen,

Copyright 1999 Girish Srinivas Gudi 


\section{ABSTRACT \\ Evaluation of Non-invasive Biomarkers for Carcinogenic \\ Exposure to Cigarette Smoke}

\section{Girish Srinivas Gudi}

Purpose. Annually 175,000 cancer deaths in the U.S. can be attributed to tobacco use. While this exposure can be avoided, many people are addicted and smoking cessation programs have relatively low success rates (20-40\%). Reducing tobacco use may be a helpful compromise where smoking cessation has failed. A noninvasive biomarker may have significant impact on smoking reduction and cessation programs by assessing exposure to the carcinogenic metabolites formed after exposure to cigarette smoke. The objective of this work was to investigate urinary 1-hydroxypyrene glucuronide (1-OHPG) and benzo[a]pyrene 7,10/8,9tetrol (BaPT) levels as non-invasive biomarkers for exposure to cigarette smoke. Methods. Volunteers, smokers ( $\geq 20$ cigarettes/day) and non-smokers ( 5 females + 6 males in each group), provided urine samples at 7:00 a.m. (overnight), 9:00 a.m., 11:00 a.m., 1:00 p.m., 3:00 p.m., 5:00 p.m., 7:00 p.m., and 9:00 p.m. Urine samples from psoriasis patients undergoing coal tar therapy were also obtained. Solid phase extraction, immunoaffinity chromatography, HPLC and synchronous fluorescence spectroscopy was used to assay BaPT and 1-OHPG. Results. BaPT levels from undetectable to $11 \mathrm{pmol} / 10 \mathrm{ml}$ were observed in samples from psoriasis patients, medicinally exposed to coal tar (mean $=4 \pm 3.4$ pmol/10ml). However, BaPT could not be detected in most samples from smokers or non-smokers in this study (limit of detection of $25 \mathrm{fmol} / 10 \mathrm{ml}$ urine). Urinary 1-OHPG levels were significantly higher among smokers (mean log 1$\mathrm{OHPG}=1.72 \pm 0.93$ ) than in non-smokers (mean log 1-OHPG $=0.80 \pm 0.92$ ) $(\mathrm{P}<0.05)$. However, significant inter-individual and temporal variability was seen both in smokers and nonsmokers $(\mathrm{P}<0.05)$. High baseline levels of 1-OHPG were seen in some nonsmokers. Conclusions. Higher assay sensitivity is required to monitor urinary levels of $\mathrm{BaPT}$ in smokers. 1-OHPG levels in urine may prove useful to monitor exposure to carcinogenic PAH in tobacco smoke. Samples obtained between 9:00 a.m. and 1:00 p.m. may be best to discern between smokers and nonsmokers. 
To Appa, Amma and Hari 
I would like to acknowledge:

Mohamadi Sarkar, Ph.D; my research advisor and mentor, for guidance, support and encouragement during the project.

Ainsley Weston, Ph.D; for guidance and help towards the completion of the project, for allowing me to use equipment in his laboratory, for providing me with samples from psoriatic patients, and the antibodies against BaPT and 1OHPG through Dr. Regina Santella.

Timothy Tracy, Ph.D; for guidance during the study. I thank him for lending me the LC-23 column heater and the SPE apparatus; for allowing me to use the vacuum evaporator in his laboratory for his useful suggestions and for accepting to be on my thesis committee.

Ann Berry, Ph.D; for guidance and accepting to be on my thesis committee.

Edwin Townsend,Ph.D; from the Dept. of Statistics at WVU for help with statistical analysis.

I take this opportunity to thank Dr. Mikael Franzon, for his helpful suggestions during the project which helped tremendously during the study design, and for providing the financial support for this project through Pharmacia-Upjohn. 


\section{TABLE OF CONTENTS}

Abstract

Dedication

Acknowledgements $\quad$ iv

Table of Contents $\quad$ V

List of Figures viii

List of Tables $\quad x$

List of Abbreviations $\quad$ xi

$\begin{array}{lll}\text { CHAPTER-I } & \text { INTRODUCTION } & 1\end{array}$

I.1 Addictive Nature and Harmful Effects of Smoking 1

I.2 Risk reduction strategies $\quad 2$

I.3 Evidence of lower relative cancer risk in light smokers 3

I.3.1 The Hammond Study 3

I.3.2 Study by Hartge et al. 3

I.3.3 The Dorn Study

I.3.4 Study by Silverman et al. $\quad 5$

$\begin{array}{ll}\text { I.4 Carcinogens in Cigarette Smoke } & 6\end{array}$

I.4.1 Benzo(a)pyrene carcinogenicity and metabolism $\quad 7$

$\begin{array}{ll}\text { I.4.2 Pyrene metabolism } & 9\end{array}$

I.5 Carcinogenesis concepts 9

I.6 Methods used to monitor exposure to cigarette smoke 12

$\begin{array}{ll}\text { I.6.1 N-Nitrosoproline in urine } & 13\end{array}$

$\begin{array}{ll}\text { I.6.2 Urinary mutagenicity } & 14\end{array}$

$\begin{array}{ll}\text { I.6.3 Measurement of the DNA adducts } & 14\end{array}$

I.6.4 Measurement of PAH and PAH metabolites in huma n urine 14 I.6.4.1 Total PAH in urine by Reverse Metabolism 15

I.6.4.2 Measurement of 3-OH BaP 15 
I.6.4.3 Measurement of 1-OHP in urine $\quad 15$

I.6.4.4 Measurement of 1-OHPG 16

I.6.4.5 Measurement of BaPT in urine $\quad 16$

$\begin{array}{ll}\text { I.7 Synchronous Fluorescence Spectroscopy } & 17\end{array}$

CHAPTER-II MATERIALS AND METHODS 19

$\begin{array}{ll}\text { II.1 Chemicals } & 19\end{array}$

$\begin{array}{ll}\text { II.2 Apparatus } & 19\end{array}$

II.3 Human subjects 20

II.4 Sample Collection and Storage 20

II.5 Analysis 21

I.5.1 Heating with concentrated $\mathrm{HCl} 21$

II.5.2 Solid Phase Extraction $\quad 21$

II.5.3 Immunoaffinity Column Chromatography 22

II.5.4 High Performance Liquid Chromatography 22

II.5.5 Synchronous Fluorescence Spectroscopy 22

II.6 Methods development 23

II.6.1 Preparation of Immunoaffinity columns from antibodies against $\mathrm{BaPT} \quad 23$

II.6.2 Extraction efficiency of immunoaffinity columns $\quad 25$

II.6.3 Determination of Retention Time of 1-OHPG 25

$\begin{array}{lll}\text { CHAPTER-III RESULTS } & 27\end{array}$

III.1 Comparison of extraction efficiency among columns using ANOVA 27

III.2 Description of study population 28

III.3 Synchronous Fluorescence Spectra 31

$\begin{array}{ll}\text { III.3a BaPT calibration curve } & 40\end{array}$

III.4 Analysis of urine samples for BaPT $\quad 41$

III.5 Samples from psoriatic patients undergoing coal-tar therapy 43

III.6 1-OHPG data 46

III.7 Statistical Methods and Analyses for 1-OHPG $\quad 47$ 
III.7.1 Normalization of data 47

III.7.2 Distribution of data 48

III.7.3 Testing for outliers $\quad 52$

III.7.4 ANOVA design $\quad 55$

III.8 Regression of 1-OHPG with the number of cigarettes smoked 67

$\begin{array}{lll}\text { CHAPTER-IV DISCUSSION } & 69\end{array}$

$\begin{array}{ll}\text { APPENDIX-I } & 76\end{array}$

A-I] Development of extraction method for BaPT using SPE cartridges $\quad 76$

A-II] Extraction of BaPT from urine sample 78

A-III] Selection of Internal Standard $\quad 78$

A-IV] Stability of the BaPT and IS 81

A-V] Optimization of the extraction 83

A-VI] Analysis of BaPT in urine from smoker and nonsmoker 85

A-VII] Evaporation and reconstitution of extract 87

A-VIII] Enzymatic deconjugation of glucuronides $\quad 88$

A-IX] Treatment with concentrated hydrochloric acid at $90{ }^{\circ} \mathrm{C} \quad 89$

A-X] Inter-conversion of BaPT and IS due to heating with $\mathrm{HCl}$

A-XI] Extraction cartridges $\quad 91$

APPENDIX-II 93

$\begin{array}{ll}\text { BIBLIOGRAPHY } & 98\end{array}$ 


\section{LIST OF FIGURES}

Fig. I-1 Metabolism of benzo[a]pyrene 8

Fig. I-2 Metabolism of pyrene to 1-OHPG 9

$\begin{array}{lll}\text { Fig.III.1 Extraction efficiency of immunoaffinity columns } & 27\end{array}$

Fig.III.2 Representative SFS in female smokers 32

Fig.III.3 Representative SFS in fema le nonsmokers 33

Fig.III.4 Representative SFS in male smokers 34

Fig.III.5 Representative SFS in male nonsmokers 35

Fig.III.6 Representative 1-OHPG SFS in coal tar treated

psoriasis patient 36

Fig.III.7 Representative BaPT SFS in coal ta $\mathrm{r}$ treated

$\begin{array}{ll}\text { psoriasis patients } & 37\end{array}$

$\begin{array}{lll}\text { Fig.III.8 Representative BaPT SFS in smokers } & 38\end{array}$

Fig.III.9 Representative BaPT SFS in nonsmokers 39

Fig.III.10 Calibration curve for BaPT in urine 40

Fig.III.11 Correlation between 1-OHPG and BaPT levels in smokers 42

Fig.III.12 Correlation between 1-OHPG and

BaPT levels in psoriasis patients $\quad 44$

Fig.III.13 Correlation between 1-OHPG and

BaPT levels in all samples $\quad 45$

$\begin{array}{lll}\text { Fig.III.14 Distribution of errors after normalization } & 47\end{array}$

Fig.III.15 1-OHPG levels in male smokers 49

Fig.III.16 1-OHPG levels in male nonsmokers 50

Fig.III.17 1-OHPG levels in female smokers 51

Fig.III.18 1-OHPG levels in female nonsmokers 52

Fig.III.19 Oulier box plots for $\log (1-\mathrm{OHPG}+1)$ data by group 54

Fig.III.20 Observed 1-OHPG levels versus predicted by the model 57

Fig.III.21 Quantile box plots and mean in smokers and nonsmokers 60

$\begin{array}{lll}\text { Fig.III.22 Smoking status and gender interaction } & 62\end{array}$ 
Fig.III.23 Mean 1-OHPG levels in smokers and nonsmokers with time 63

Fig.III.24 Mean 1-OHPG levels males and female with time 64

Fig.III.25 Mean 1-OHPG levels males smokers and nonsmokers with time 65

Fig.III.26 Mean 1-OHPG levels females smokers and nonsmokers with time 66

Fig.III.27 Correlation between Log(1-OHPG) levels and number of cigarettes $\quad 68$

Fig. A.1 Chromatograph of BaPT extracted using Sep-pak cartridges 77

Fig. A.2 Chromatograph of BaP-7,10/8,9-Tetrol 80

Fig. A.3 Chromatograph of BaP-7/8,9,10-Tetrol 81

Fig. A.4 Chromatograph of BaPT and IS 83

Fig. A.5 Chromatograph of BaPT and IS in spiked urine 86

Fig. A.6 Chromatograph of extracted nonsmoker urine 87 


\section{LIST OF TABLES}

Table I-1 Lung cancer mortality ratios from Hammond study

Table I-2: $\quad$ Estimated Relative Risk (RR) of bladder cancer according to duration, dose rate, and smoking status

Table I-3 Mortality ratios for specific underlying causes of death by smoking level 5

Table III-1 Volunteer data 30

Table III.2 BaPT and 1-OHPG levels from sm okers 41

Table III.3 BaPT and 1-OHPG levels from psoriasis patients 43

Table A.1 Percent Extraction using Sep-pak 77

$\begin{array}{lll}\text { Table A.2 Percent Extraction from spiked urine } & 78\end{array}$

$\begin{array}{lll}\text { Table A.3 Selection of internal standard } & 79\end{array}$

Table A.4 A/IS ratio at room temperature 82

Table A.5 Optimization of extraction 84

Table A.6 A/IS ratio in urine from smoker and nonsmoker 85

Table A.7 A / IS ratio in urine after concentration 88

Table A.8 A / IS ratio in urine after heating with $\mathrm{HCl} 90$

TableA.9 Extraction efficiency of various columns 92 


\section{List of Abbreviations}

$\begin{array}{lll}\text { 1-OHPG } & = & \text { 1-hydroxypyrene glucuronide } \\ 1-\mathrm{OHP} & = & 1 \text {-hydroxypyrene } \\ \mathrm{BaPT} & = & \text { Benzo[a]pyrene-r-7, } \mathrm{t}-8, \mathrm{t}-9, \mathrm{c}-10 \text {-tetrahydrotetrol }( \pm) \\ \mathrm{IS} & = & \text { Benzo[a]pyrene-r-7, } \mathrm{t}-8, \mathrm{t}-9, \mathrm{t}-10 \text {-tetrahydrotetrol }( \pm) \\ \mathrm{SFS} & = & \text { Synchronous Fluorescence Spectroscopy } \\ \mathrm{IAC} & = & \text { Immunoaffinity chromatography } \\ \mathrm{LSN} & = & \text { Least significant number }\end{array}$




\section{CHAPTER-I}

\section{INTRODUCTION}

\section{I.1 Addictive Nature and Harmful Effects of Smoking:}

The U.S. Department of Health and Human Services identified tobacco use as nicotine addiction and concluded that "cigarettes and other forms of tobacco are addictive. Nicotine is the drug in tobacco that causes addiction. The processes that determine nicotine addiction are similar to those that determine addiction to drugs such as heroin and cocaine." 1 Drug dependence involves the repeated administration of a substance that contains a psychoactive chemical. Like other drug dependencies, nicotine dependence is a progressive, chronic, relapsing disorder that is characterized by stereotypic patterns of use, use despite harmful effects, and relapse following abstinence and recurrent drug cravings. Tobacco use produces tolerance and physical dependence. An interplay exists between the addictive nature of cigarettes and their behaviorally reinforced properties. Over time, smoking becomes associated with a number of cues that reinforce the behavior. ${ }^{2}$

According to the American Cancer Society, in the year 1999, about 563,100 Americans are expected to die of cancer (more than 1,500 people a day). About 175,000 of these cancer deaths are attributable to tobacco use. ${ }^{3}$ Cigarette smoking is the largest preventable cause of disease and disability in the United States. Smoking has been associated with diseases such as cancer, chronic obstructive pulmonary disease, coronary heart disease, and stroke. About $80-90 \%$ of lung cancer, the most important cause of cancer death in the world, is attributable to cigarette smoke. In addition to lung cancer, smoking has been accepted as the major cause of cancers of the larynx, oral cavity, and esophagus, and is a contributory factor in cancers of the pancreas, bladder, kidney, stomach and uterine cervix. Environmental tobacco smoke (ETS, also known as passive or involuntary smoking) is also a human carcinogen and is causally associated with lung cancer in adults. ${ }^{4}$ 
Clearly, the best way to reduce the risk of cancer from tobacco smoke is to quit smoking. Smoking cessation has substantial health benefits and dramatically reduces the risk of most smoking-related diseases. Unfortunately, while the risks of smoking are well-known and despite mass media campaigns and efforts of the medical community against smoking, more than 25 percent of the people in the United States of America continue to smoke. ${ }^{5}$ Survey data have shown that majority of smokers have made at least one serious but unsuccessful attempt to stop smoking. ${ }^{6}$ Almost two out of five smokers have made three or more attempts to quit. ${ }^{7}$ Horn D. reported that between 1970 and 1975, 84 percent of U.S. smokers thought seriously about giving up smoking, and 68 percent of these smokers tried to quit. ${ }^{8}$ Once addicted to tobacco it is difficult to quit. It is the combination of physiologic and psychological dependence that makes cessation such a challenge. ${ }^{9}$

\section{I.2 Risk reduction strategies:}

Various methods have been used for smoking cessation including medication, nicotine chewing gum and dermal patches, hypnosis, acupuncture, physician advice and counseling, educational approaches, mass media, community programs and behavioral methods. ${ }^{10}$ However, most smokers prefer "do it yourself" (self-help) and minimal contact strategies over formal treatment programs, and in fact, 90 percent of U.S. ex-smokers have quit on their own, without the benefit of any formal treatment program. ${ }^{11}$ Success rate of most formal smoking cessation programs is rather low (in the range of 20-25\%).

Heavy smokers are at a high risk of developing smoking related diseases; a cessation of smoking would therefore be highly effective in reducing this risk. However, Ockene JK et al. have shown that light smokers, in comparison to heavy smokers, are more likely to quit and twice as likely to stay abstinent for longer periods of time. ${ }^{12}$ In view of these facts Millatmal $\mathrm{T}$ et al. have suggested that smoking reduction may represent an alternative to cessation in order to 
reduce the risks of smoking related diseases for those heavy smokers who are unable to quit completely. ${ }^{13}$

\section{I.3 Evidence of lower relative cancer risk in light smokers:}

Overwhelming evidence in literature suggests that moderate or light smokers are at a lower relative risk of developing cancer in comparison to heavy smokers:

\section{I.3.1 The Hammond Study:}

In a study, involving 440,558 men and 562,671 women, Hammond E.C. reported the lung cancer mortality rates for men and women smoking different number of cigarettes. ${ }^{14}$ Table I-1 shows results from this study.

Table I-1 : Lung cancer mortality ratios from Hammond study.

\begin{tabular}{|l|l|l|}
\hline & $\begin{array}{l}\text { Current Number of } \\
\text { cigarettes a day }\end{array}$ & Mortality Ratio \\
\hline MEN & & \\
\hline & $1-9$ & 4.6 \\
\hline & $10-19$ & 7.48 \\
\hline & $20-39$ & 13.14 \\
\hline & $40+$ & 16.61 \\
\hline WOMEN & & \\
\hline & $1-19$ & 1.06 \\
\hline & $20+$ & 4.76 \\
\hline
\end{tabular}

This study reported increase in mortality rates with the number of cigarettes smoked per day, degree of inhalation and age when they began smoking.

\section{I.3.2 Study by Hartge et al. :}

In a large multi-center study conducted by the National Cancer Institute, involving data from 8,764 subjects, Hartge et al. studied the contribution of various aspects of a person's cigarette smoking history to his increased risk of bladder cancer. ${ }^{15}$ Table I-2 presents the findings of the study when both average 
cigarette dose per day and duration of smoking are cross-classified for current and former smokers.

Table I-2: Estimated Relative Risk (RR) of bladder cancer according to duration, dose rate, and smoking status.

\begin{tabular}{|c|c|c|c|}
\hline Category \&Dose & \multicolumn{3}{|c|}{ Duration of smoking } \\
\hline & $<20 \mathrm{yr}$ & $20-39 \mathrm{yr}$ & $\geq 40 \mathrm{yr}$ \\
\hline & & & \\
\hline Current smokers & & & 2.7 \\
\hline$<20 /$ day & 1.7 & 1.6 & 3.1 \\
\hline & $(1.0-2.7)$ & $(1.2-2.2)$ & 3.8 \\
\hline $20-39 /$ day & 2.2 & 3.8 & 3.8 \\
\hline & $(1.3-3.7)$ & $(3.0-4.6)$ & $(2.8-5.0)$ \\
\hline$\geq 40 /$ day & 2.4 & 4.0 & \\
\hline & $(1.1-5.4)$ & $(2.7-5.9)$ & 1.9 \\
\hline & & & $(1.4-2.6)$ \\
\hline Former smokers & & & 2.5 \\
\hline$<20 /$ day & 1.3 & 1.5 & $(2.0-3.2)$ \\
\hline & $(1.1-1.7)$ & $(1.2-1.9)$ & 2.8 \\
\hline $20-39 /$ day & 1.4 & 1.8 & $(2.0-3.8)$ \\
\hline & $(1.1-1.9)$ & $(1.5-2.2)$ & 2.1 \\
\hline
\end{tabular}

Estimates are adjusted for age, sex, and race. Risk is relative to 657 cases and 2,198 controls who never smoked. $95 \%$ CIs are shown in parentheses.

The study reported that the estimated increase in bladder cancer risk for current smokers was $0.9 \%$ for each additional year of smoking $(\mathrm{P}=0.073)$ and $1.4 \%$ for each additional cigarette smoked daily $(\mathrm{P}<0.001)$. The corresponding estimates for former smokers were $1.4 \%$ for each additional year $(\mathrm{P}=0.000)$ and $0.3 \%$ for each additional cigarette $(\mathrm{P}=0.224)$. 


\section{I.3.3 The Dorn Study:}

Dorn H.F initiated a study involving a population of over 293,000 life insurance policyholders with a follow-up period of eight and a half years. Kahn H.A. reported mortality rates from the Dorn study by smoking level with the underlying cause of death. ${ }^{16}$ Table I-3 shows the results from this study.

Table I-3: Mortality ratios for specific underlying causes of death by smoking level.

\begin{tabular}{|l|c|c|}
\hline \multicolumn{1}{|c|}{ Cause of Death } & \multicolumn{2}{|c|}{ Current Cigarette Smokers } \\
\hline $\begin{array}{l}\text { Cancer of lung \& } \\
\text { bronchus }\end{array}$ & $\mathbf{1 0 - 2 0 / d a y}$ & $\mathbf{2 1 - 3 9 / d a y ~}$ \\
\hline Cancer of larynx & 9.05 & 16.93 \\
\hline $\begin{array}{l}\text { Cancer of mouth, } \\
\text { pharynx or esophagus }\end{array}$ & 8.33 & 9.39 \\
\hline
\end{tabular}

Mortality ratios due to cancer were lower in people who smoked lesser number of cigarettes per day in comparison to heavy smokers suggesting that light or moderate smokers are less likely to die from cancer as compared to heavy smokers.

\section{I.3.4 Study by Silverman et al. :}

It has been proposed that upper aerodigestive tract carcinogenesis is a process of 'field cancerization' 17 which involves repeated exposure of a region's entire tissue area to carcinogenic insult which increases the tissue's risk for developing multiple independent premalignant and malignant foci. Second primaries are the major threat to long-term survival after successful therapy of early-stage head and neck squamous cell carcinoma (HNSCC). The concept of field carcinogenesis may explain the clinical occurrence of multiple primary and second primary tumors in HNSCC. Second primaries develop at a constant rate of $4-7 \%$ of treated patients per year and are not treatment related. 
In a follow-up study of 1 to 15 years, by Silverman et al. ${ }^{18}, 160$ head and neck carcinoma patients were studied. Of these, 117 were tobacco users (90\% of which were cigarette smokers) and 43 were tobacco nonusers. Occurrence of second primary cancer in smokers whose smoking habits remained unchanged was $30 \%$ compared with $15 \%$ for those reducing smoking and $13 \%$ for those who completely stopped smoking. Second primary cancer in tobacco nonusers was $16.3 \%$. Reducing the smoking habit resulted in similar effect as seen in those who completely quit smoking or in people who never smoked at all. This observation suggests that there is the possibility of a critical carcinogenic threshold for smoking, beyond which the risk of developing second primary head and neck cancer increases rapidly as compared to nonsmokers.

\section{I.4 Carcinogens in Cigarette Smoke:}

Cigarette smoke contains more than 3800 identified chemical constituents, of which more than 40 are accepted carcinogens. ${ }^{19}$ Carcinogens in tobacco and tobacco smoke are:

Polycyclic Aromatic Hydrocarbons (PAH) such as benzo[a]pyrene, benzo[a]anthracene, benzofluoranthenes, chrysene etc.

Nitrosamines such as N-nitrosodimethylamine, N-nitrosoethylmethylamine, Nnitrosodiethylamine, N-nitrosodiethanolamine, N-nitrosonornicotine(NNN), 4(methylnitrosoamino)-1-(3-pyridyl)-1-butanone [NNK] etc.

NNK is formed from nicotine during the processing of tobacco and during smoking, and is a potent lung carcinogen in laboratory animals independent of the route of administration.

Aza-arenes such as quinoline, dibenz[a,h]acridine, $7 \mathrm{H}$-dibenzo[c,g]carbazole.

Aromatic amines such as 2-toluidine, 2-naphthylamine, 4-aminophenyl

Aldehydes such as formaldehyde, acetaldehyde and crotonaldehyde.

Miscellaneous organic compounds such as benzene, vinyl chloride, acrylonitrile, 1,1-dimethylhydrazine, 2-nitropropane, ethylcarbamate. 


\section{I.4.1 Benzo(a)pyrene carcinogenicity and metabolism:}

Polycyclic aromatic hydrocarbons (PAH) such as benzo[a]pyrene (BaP), which are formed by the incomplete combustion of tobacco during smoking, are well recognized carcinogens that have been shown to induce tumors of the lung in laboratory animals exposed by inhalation, instillation in the trachea, or implantation in the lung. The content of benzo[a]pyrene in processed tobacco can be as high as $90 \mathrm{ng}$ per gram of tobacco. Mainstream smoke from cigarettes contains about 20-40 ng of benzo[a]pyrene per cigarette ${ }^{20}$. Thus, the dose of benzo[a]pyrene for a person who smokes a pack of twenty cigarettes a day is about 400-800 ng. Habitual smokers are thus exposed to large doses of $\mathrm{BaP}$ on a regular basis through cigarette smoke. Considering the amounts of these compounds in cigarette smoke and their carcinogenic potency in experimental animals, it is believed that they play a role in carcinogenesis due to smoking. PAH are not only potent procarcinogens but also potent enzyme inducers. They induce their own metabolism as well as the metabolism of other drugs including antipyrine, theophylline ${ }^{21}$. Components of diet, especially smoked, charbroiled or grilled foods are known to contain significant amounts of PAH. On a single occasion of consuming such a PAH-rich diet the dose of BaP received may be as high as 1000 ng. ${ }^{22}$ Humans are exposed to about $10-20$ ng of BaP every day due to environmental air exposure.

Metabolism of $\mathrm{BaP}$ has been studied extensively, the primary metabolites being three epoxides: the 4,5-epoxide, 7,8-epoxide, and the 9,10-epoxide. Several phenols, quinones and dihydrodiols have also been identified. The epoxides can be conjugated to glutathione S-conjugates and the phenols and diols can be conjugated to water-soluble compounds by either sulfate conjugation or glucuronide conjugation.

The (-)-trans-7,8-diol is converted to two stereoisomers of 7,8-diol-9,10epoxides: diol epoxide I and diol epoxide II. Each diol epoxide is hydrolyzed to two tetrols and reduced to one triol. Diol epoxide I is hydrolyzed to 7,10,/8,9- 
tetrahydroxytetrahydro $\mathrm{BaP}$ and 7/8,9,10-tetrahydroxytetrahydro $\mathrm{BaP}$ and reduced to 7/8,9-trihydroxy-pentahydro $\mathrm{BaP}$. Diol epoxide II is hydrolyzed to 7,9,10/8-tetrahydroxytetrahydro $\mathrm{BaP}$ and 7,9/8,10-tetrahydroxytetrahydro $\mathrm{BaP}$ and reduced to 7,9/8-trihydroxy-pentahydro BaP. (shown in figure I.1)

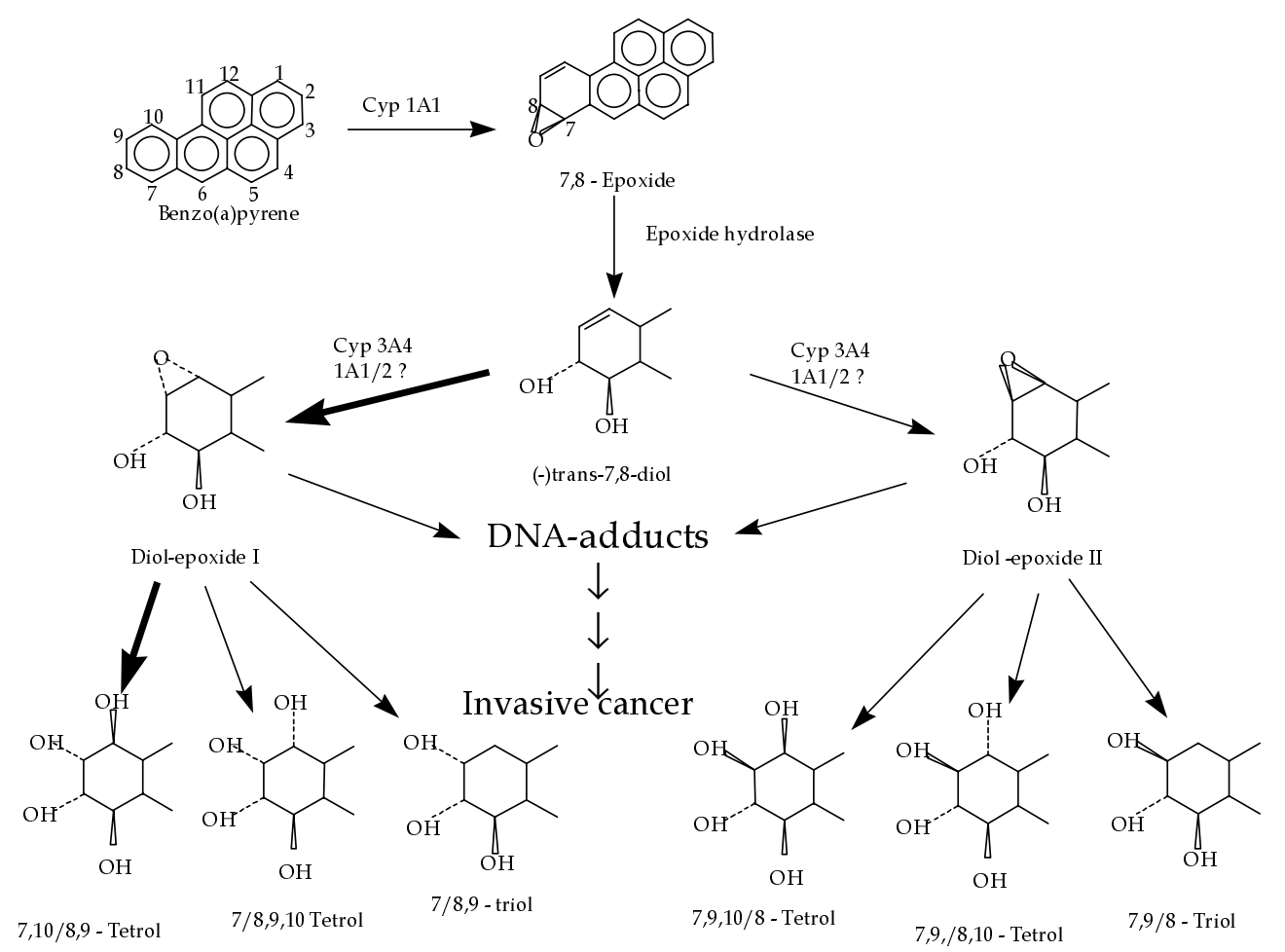

Fig. I-1 Metabolism of benzo[a]pyrene

The $\mathrm{BaP}$ diol-epoxides are the most carcinogenic/mutagenic metabolites of $\mathrm{BaP}$; they are highly reactive and react with macromolecules such as protein and DNA. Bio-monitoring of $\mathrm{BaP}$ diol epoxide $\mathrm{I}$, the ultimate carcinogen of $\mathrm{BaP}$, would be the best way to assess the carcinogenic outcome from cigarette smoke. Unfortunately, in aqueous solutions, the diol epoxides are very unstable and have a half-life of less than 10 minutes making the measurement of $\mathrm{BaP}$ diol epoxides impractical. ${ }^{23,24}$ Assay of BaPT, the major tetrol from hydrolysis of the BaPT diol-epoxide I, would be a good indicator of carcinogenic exposure from 
cigarette smoke and would be useful in estimation of the internal dose of the ultimate carcinogen (BPDE-I). A number of metabolic pathways activate or detoxify carcinogens and procarcinogens. Genetic polymorphism in activating enzymes such as CYP1A1, CYP2D6, and in detoxifying enzymes such as Nacetyltransferases, glutathione-S-transferases are thought to be determinants of cancer susceptibility.

\section{I.4.2 Pyrene metabolism:}

Pyrene is a PAH which is present in complex smoke mixtures. Pyrene intake through mainstream smoke is estimated to be in the range of $0.56-33.9$ nmol/day. ${ }^{25}$ Its levels have been measured in various foods such as roasted meats, various vegetables like spinach and kale, fish, cereals, and sweets.

It is metabolized primarily to 1-hydroxy pyene (1-OHP), which is excreted as 1hydroxy pyrene glucuronide conjugate in urine and faeces (fig I-2). It is also excreted as the 1,2-dihydroxy derivative.<smiles></smiles>

Pyrene

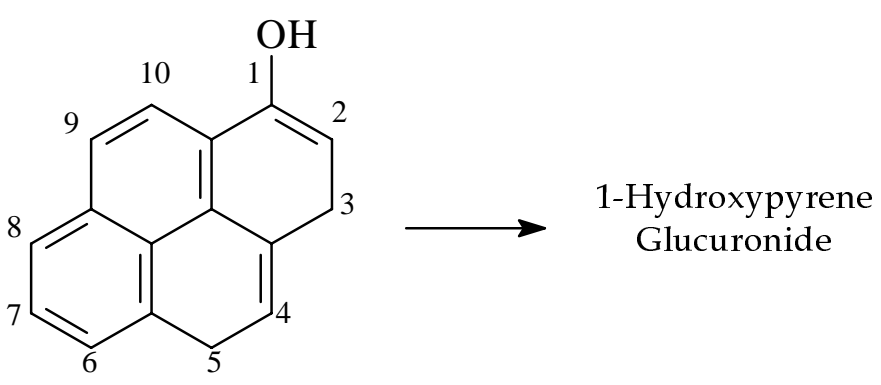

1-Hydroxypyrene

Fig. I-2 Metabolism of pyrene to 1-OHPG

\section{I.5 Carcinogenesis concepts:}

Cigarette smoke contains more than 40 carcinogenic chemicals; cancer due to smoking can thus be considered as a form of chemical carcinogenesis. Current information suggests that chemical carcinogenesis is a multistage process. The existence of the sequential stages of carcinogenesis first became apparent in connection with rabbit skin ${ }^{26}$ and mouse skin ${ }^{27,28,29}$ response. One of the best- 
studied models is the two-stage carcinogenesis (initiation-promotion) system in mouse skin. In this model, benign tumors (papillomas) and malignant lesions (squamous cell carcinomas) can be induced either by a single large dose or by multiple fractionated, doses of a complete carcinogen. Complete carcinogenesis in mouse skin refers to the production of tumors after repeated application of a carcinogen. Compounds possessing both, tumor initiating activity and tumor promoting activity will induce tumors in this regimen. Skin tumors can also be induced by the sequential application of a subthreshold dose of a carcinogen (initiation phase) followed by repetitive treatment with a noncarcinogenic tumor promoter (promotion phase). Initiation involves the induction of an irreversibly altered cell and is frequently equated with a mutational event. The initiation phase thus requires only a single application of either a direct or indirect carcinogen at a subthreshold dose and is essentially irreversible. Promotion is the experimentally defined process by which the initiated cell clonally expands into a visible tumor, often a benign lesion such as a papilloma. The promotion phase is brought about by repetitive treatments after initiation and is initially reversible but later irreversible. ${ }^{30}$ The generality of two-stage tumor induction has been demonstrated in a number of experimental carcinogenesis systems like liver, bladder, lung, colon, esophagus, stomach, mammary gland, pancreas, and lung cells in culture. ${ }^{31}$ The two-stage model of initiation and promotion for chemical carcinogenesis has provided knowledge of the qualitatively different mechanisms by which chemicals can act. The end products of tumor promotion are generally benign lesions or foci of preneoplastic cells. The progression of benign tumors to malignant cancers is a phase in carcinogenesis clearly distinct from promotion. ${ }^{32}$ Progression involves the conversion of tumors from benign to malignant and can be considered an open-ended process since tumors may continue to increase in their degree of malignancy and heterogeneity.

Genotoxic carcinogens have high chemical reactivity (such as alkylating agents) or can be metabolized to reactive intermediates by the host. They form 
covalent adducts with macromolecules and target DNA in the nucleus and mitochondria. There is a good correlation between the ability to form DNA adducts and the potency to induce tumors in laboratory animals; hence DNA is considered the ultimate target for most carcinogens. Carcinogen-DNA adduct formation is recognized as a necessary step in the development of most chemicalinduced cancers. ${ }^{33}$ However, DNA adducts are generally considered to be necessary but not sufficient for tumorigenesis. ${ }^{34}$

Two classes of genes, proto-oncogenes and tumor-suppressor genes, are involved in the evolution of most, if not all, cancers. Proto-oncogenes encode proteins that are involved in the control of cell growth. Alteration of the structure and/or expression of proto-oncogenes can activate them to become oncogenes capable of transforming susceptible cells into the neoplastic phenotype. Proto-oncogenes can be classified (based on the functional and biochemical properties of their protein products) as growth factors (e.g. Sis and int-2), growth factor receptors (e.g. erb B, fms), signal transducers (e.g. abl, src, raf, H-ras, K-ras, N-ras), transcription factors (e.g. erb A, ets, fos, jun), and programmed cell death regulators (e.g. bcl-2). Activation of oncogenes confers a growth advantage to the cell. Mutations such as base substitutions, deletions and insertions are capable of activating proto-oncogenes. Gene amplification can result in a selective advantage for cell growth. Chromosomal rearrangements can lead to hematologic malignancy by the transcriptional activation of protooncogenes or the creation of fusion genes. Many properties of neoplastic cells including immortality and tumorigenicity appear to result from the inactivation or loss of function of normal cellular genes. These cellular genes, hypothesized to regulate cellular proliferation and growth in a negative fashion, are termed tumor suppressor genes. A large number of tumor suppressor genes are hypothesized to exist; however, only few have been identified. Products of some tumor suppressor genes may act directly to oppose the function of oncogenes, while others may interact indirectly with oncogenes. Examples of tumor 
suppressor genes include RB1, p53, WT1, APC, p16, NF1, NF2, VHL, BRCA1 and BRCA2. Wild type tumor suppressor genes inhibit transformation by oncogenes whereas mutant tumor suppressor genes are capable of functioning as oncogenes. Mutated alleles of the genes of the tumor suppressor genes mentioned above are present in individuals with specific inherited predisposition to cancer.

Normal cells are not neoplastically transformed by a single oncogene but rather require two or more co-operating oncogenes and inactivation of multiple tumor-suppressor genes supporting a multistep or multihit model of carcinogenesis. The number of genes altered in a cancer cell compared to a normal cell is not known, evidence suggests that 3-10 genetic events are involved in common adult human malignancies. Analysis of multistep carcinogenesis at the molecular level indicates that the process of carcinogenesis is significantly more complicated than the relatively simple two-stage (initiation-promotion) model or the three-stage model of initiation, promotion and progression. ${ }^{35}$

At least multiple mutations must occur for a tumor cell to arise, these mutations may occur by different genetic mechanisms, thus a single toxicological end point may not correlate with carcinogenic potency of chemicals. Steady state DNA adduct levels most frequently increase linearly with dose. The formation of tumors is also dependent of a number of factors including the proliferative capacity of the target tissue, the sex of the animal, genotoxic properties of the specific adducts formed, the influence of promoters and other unknown events. ${ }^{36}$

\section{I.6 Methods used to monitor exposure to cigarette smoke:}

Various indirect methods have been developed to estimate human exposure to tobacco related carcinogens including analysis of personal air samples $^{37}$, analysis of food ${ }^{38}$, estimation of carcinogen content in cigarettes ${ }^{39}$, human environmental exposure ${ }^{40}$ and using these values for the determination of the potential dose ${ }^{41}$. 
Carcinogen intake from cigarettes may vary considerably depending on the tar content of the cigarette, depth of inhalation, number of puffs taken and the distance from the filter until which the cigarette is smoked. Estimated dose calculated from exposure assessment does not take into account the bioavailability factor due to lung deposition and exhalation but represents the intake or the potential amount that could be absorbed if the bioavailability was $100 \%$. Estimation of the carcinogen dose received by smokers using values obtained from the content of cigarettes may not be very reliable because smoking habits differ greatly in people. Internal dose of the ultimate carcinogen in individuals would vary with the same dose of the carcinogen because of interindividual differences in metabolism. Hence for determining the carcinogenic exposure from tobacco use it is important to monitor the carcinogenic compounds from tobacco or their metabolites at or near the target site.

Various methods have been used to measure the extent to which tobacco smoke is inhaled and taken into the human body. Constituents of tobacco smoke such as carbon monoxide $(\mathrm{CO})$, nicotine and hydrogen cyanide $(\mathrm{HCN})$ are used routinely to measure exposure. However, they are not necessarily adequate markers of toxic/carcinogen exposure.

\section{I.6.1 N-Nitrosoproline in urine:}

The endogenous formation of $\mathrm{N}$-nitrosamines in humans following ingestion of a source of nitrate and of proline has been demonstrated. Tobacco smoke contains up to $600 \mathrm{pg}$ of nitrogen oxides per cigarette and several $\mathrm{mg}$ of nitrosatable amines. Salivary thiocyanate, which is increased by smoking, is known to be an effective nitrosation catalyst and inhalation of tobacco smoke may thus increase endogenous formation of $\mathrm{N}$-nitrosamines in smokers. In a study by Hoffmann and Brunnemann ${ }^{42}$, smoking and nonsmoking men were placed on a controlled diet. The 24-h urinary excretion of N-nitrosoproline (NPRO) was significantly higher in smokers than in nonsmokers; addition of proline to the diet increased NPRO excretion significantly in smokers but not in nonsmokers. 


\section{I.6.2 Urinary mutagenicity:}

Mutagenic activity is usually detectable only after urine has been concentrated and treated with deconjugating enzymes, such as $\beta$-glucuronidase and arylsulphatase or a rat hepatic microsomal fraction (S9). The most frequently used biological indicators of mutagenicity have been the bacterial strains routinely applied in mutagenicity testing; the best indicator strains for urinary mutagenicity related to smoking are the frame-shift mutation strains (TA98 and TA1538) of S. typhimurium. The test system is sensitive to a marker amino acid (histidine) in concentrated urine samples. Clonfero et al.43 have used the urinary mutagenesis assay for biomonitoring for PAH exposure.

\section{I.6.3 Measurement of the DNA adducts:}

DNA adduct measurement in biological samples proves to be a good biomarker for PAH induced carcinogenesis. A number of methods have been developed for sensitive detection of carcinogen-DNA adducts in humans. These include enzyme immunoassays ${ }^{44},{ }^{32} \mathrm{P}$-postlabelling assay ${ }^{45}$, gas chromatography/mass spectrometry ${ }^{46}$, fluorescence spectroscopy ${ }^{47}$, electrochemical conductance ${ }^{48}$. Weston, $\mathrm{A}^{49}$ has summarized the techniques used to quantify DNA adducts in humans. DNA-adduct measurement techniques have been applied for the evaluation of tobacco induced DNA damage in various human tissues including oral mucosa ${ }^{50}$, lungs ${ }^{51}$, cervical biopsies ${ }^{52}$ etc. Enzyme immunoassays such as ELISA and USERIA with an antibody raised against BaP-DNA have been developed ${ }^{53}$. DNA adducts specific to NNK and NNN have been detected in smokers' lungs. Such measurement from biological target tissue suffers from the drawback of being an invasive method and may not find application as a routine biomonitoring technique.

\section{I.6.4 Measurement of PAH and PAH metabolites in human urine:}

Urinary metabolite measurement techniques are non-invasive unlike the various techniques for PAH-DNA measurement in tissue or blood. 
I.6.4.1 Total PAH in urine by Reverse Metabolism: Difficulties in analyzing PAH in body fluids arise from the fact that humans may be exposed to a large number of different PAH and each of these $\mathrm{PAH}$ is converted to a large number of metabolites. To overcome this problem Becher et al. ${ }^{54}$ developed an analytical procedure which involved extraction of $\mathrm{PAH}$ and their metabolites from urine using commercial C-18 modified silica cartridges, reduction of metabolites to parent PAH using hydriodic acid and subsequent analysis by HPLC and fluorescence detection. This approach may have value in assessing the dose of $\mathrm{PAH}$ received by a person but it gives no insight to the carcinogenic risk from the metabolites of procarcinogenic PAH which may be the ultimate carcinogens. It does not take into account the inter-individual differences in metabolism and the method does not utilize techniques having high specificity for detection of the PAH.

I.6.4.2 Measurement of 3-OH BaP: 3-hydroxy benzo[a]pyrene, a metabolite of benzo[a]pyrene, has been measured at low levels $(0.5-20 \mathrm{ng} / \mathrm{L})$ in urine of coke oven workers exposed to PAH using sophisticated laser-induced fluorescence and laser-excited Shpol'skii techniques. ${ }^{55}$ The 3-OH BaP concentrations in control urine samples were correlated with the 1-OHP concentrations $\left(\mathrm{r}^{2}=0.89\right)$.

I.6.4.3 Measurement of 1-OHP in urine: Jongeneelen et al. ${ }^{56}$ suggested 1hydroxypyrene as a biomarker of human exposure to PAH. Pyrene is always present in PAH mixtures and is mainly metabolized to the intermediary 1-OHP to form 1-OHP -glucuronide which is excreted in urine. ${ }^{57}$ Using fractionated human liver as metabolic system, the activation of PAH in crude coal tar to mutagenic metabolites was closely correlated to the simultaneous formation of 1OHP ${ }^{58}$. Thus the formation of 1-OHP is a good indicator for the activation of pre-mutagens from crude coal tar. Urine of psoriatic patients, undergoing dermal treatment with coal tar solutions, yielded a highly increased urinary concentration of 1-OHP and showed an increased response in the salmonella/mutagenicity assay; also, urinary 1-OHP and mutagenic metabolites 
were correlated. ${ }^{59}$ Roggi et al. ${ }^{60}$ reported smoking to be a major factor influencing urinary 1-OHP levels. Multiple regression analysis of log transform of 1-OHP excretion data reported by Boogard et al. ${ }^{61}$ and Moen et al. ${ }^{62}$ had a significant P-value for smoking status as one of the determinants. Gilbert et al. ${ }^{63}$ and Merlo et al. ${ }^{64}$ reported significantly increased levels of urinary 1-OHP in cigarette smokers as compared to nonsmokers.

I.6.4.4 Measurement of 1-OHPG: Strickland et al.65 used an analytical method utilizing immunoaffinity chromatography, HPLC, SFS, GC-MS to measure pyrene metabolites in human urine prior to enzymatic deconjugation. They found the major fluorescent metabolite in immunoaffinity purified urine to be more polar than 1-OHP and BaP tetrols and diols. Following digestion with $\beta$ glucuronidase, this metabolite co-chromatographed with authentic 1-OHP and exhibited fluorescence spectra characteristic of 1-OHP, suggesting that the metabolite was a glucuronide conjugate of 1-OHP. The identity of 1hydroxypyrene glucuronide (1-OHPG) was further confirmed by GC-MS analysis of the trimethylsilyl derivative. 1-OHPG was found to be approximately 5 times more fluorescent than 1-OHP and thus proves to be a more sensitive biomarker for assessing exposure to pyrene in mixtures of PAHs. Kang et al.66 measured the 1-OHPG levels (quantitated in terms of 1-OHP) and PAH-DNA adduct levels in WBC in non-smoking males who consumed charbroiled beef for five consecutive days. The individual mean level of PAH-DNA adducts in peripheral blood cells during and after the feeding period correlated with mean concentration of urinary 1-OHPG in the same individuals.

I.6.4.5 Measurement of BaPT in urine: Uziel et al ${ }^{67}$ detected BaP-7,10/8,9-tetrol $(\mathrm{BaPT})$ in human urine by direct synchronous fluorescence detection of diluted urine. This technique, without a separation step before analysis may have a lot of interference with other metabolites containing the pyrene moiety. They also noticed a quenching effect due to other substances present in urine. Because of the complexity of urine as an analytical matrix, it is necessary to have a 
separation step such as HPLC to separate out BaPT from other interfering substances prior to analysis by synchronous fluorescence spectroscopy (SFS). Weston et al. ${ }^{68}$ developed a method involving immunoaffinity column chromatography (IAC), HPLC and SFS for analysis of BaPT in human urine. BaPT levels of 0.24-3.12 pmol per $\mathrm{ml}$ urine were detected in non-smoking subjects who had consumed a diet rich in char-broiled food. The limit of detection was $0.01 \mathrm{pmol} / \mathrm{ml}$ given $10 \mathrm{ml}$ urine. Weston et al. ${ }^{69}$ measured the $\mathrm{BaPT}$ and 1-OHPG in psoriasis patients undergoing Goeckerman therapy. Urinary levels of $\mathrm{BaPT}$ in these patients, excreting large amounts of $\mathrm{BaP}$ and pyrene metabolites due to topical application of coal tar, varied from $<10$ to 330 $\mathrm{fmol} / \mathrm{ml}$ compared to $<10$ to $40 \mathrm{fmol} / \mathrm{ml}$ in non-coal tar treated controls. They reported urinary levels of 1-OHPG (quantitated in terms of 1-OHP) to be 180 $50,000 \mathrm{fmol} / \mathrm{ml}$ in coal tar treated patients.

\section{I.7 Synchronous Fluorescence Spectroscopy:}

Conventional fluorescence spectroscopy usually seeks an excitation or emission spectrum. Fluorescence measurements made with both excitation and emission monochromators fixed (dual fixed wavelength) have very little specificity. Specificity is increased by using synchronous fluorescence technique where both the excitation and emission monochromators are driven simultaneously with a fixed wavelength difference. This approach greatly simplifies the spectrum because if the fixed wavelength difference used is equal to the distance between the longest 0,0 transition in the excitation spectrum and the shortest 0,0 transition in the emission spectrum (ie. The Stoke's shift referred to as $\Delta \lambda$ ) then a fluorescence signal will only be observed when the two maxima are met. When the wavelength difference is set at $34 \mathrm{~nm}$ both 1-OHPG and BaPT show a sharp excitation maximum at $346 \mathrm{~nm}$ which is characteristic of the pyrene moiety.

The objective of this study was to evaluate BaPT and 1-OHPG as noninvasive biomarkers of toxic exposure to $\mathrm{PAH}$ from cigarette smoke. BaPT 
and 1-OHPG levels were measured in urine samples obtained from nonoccupationally exposed smoking and nonsmoking volunteers during the day while on a normal diet not consuming any PAH rich foods. The levels obtained were analyzed for smoking effect. Male as well as female volunteers were studied to investigate any gender-related differences. Multiple samples were collected on the same day at different times to identify changes in the BaPT and 1-OHPG levels during the day. Since BaPT levels were not detected in most of the samples from either the smoker or nonsmoker group a set of urine samples from psoriasis patients, medicinally exposed to large amounts of PAH due to coal-tar therapy were included in the study. Psoriasis patients undergoing Goeckerman therapy have been shown by Weston et d $[.69$ to excrete larger amounts of benzo[a]pyrene and pyrene metabolites than untreated individuals. 


\section{CHAPTER-II}

\section{MATERIALS AND METHODS}

\section{II.1 Chemicals:}

Benzo[a]pyrene-r-7, t-8,t-9,c-10-tetrahydrotetrol ( \pm ) and benzo[a]pyrene-r-7, t-8,t9,t-10-tetrahydrotetrol $( \pm)$ were obtained from the NCI Chemical Carcinogen Reference Standard Repository (Kansas City, Missouri - 64110, USA). Polyethylene glycol (average molecular weight 3,350), dimethyl suberimidate, boric acid, triethanolamine and sodium azide were obtained from Sigma Chemical Company (St.Louis, Missouri - 63178, USA). Glycine (electrophoresis purity reagent) was obtained from Bio-Rad Laboratories (Richmond, California 94804, USA). Tris(hydroxymethyl) methylamine (AnalaR) was obtained from Hoefer Scientific Instruments (San Francisco, California - 94107, USA). Protein A Sepharose 4 Fast Flow was from Pharmacia Biotech (Piscataway, New Jersey 08855, USA). Sep-Pak ${ }^{\circledR}$ C-18 solid phase extraction cartridges with a reservoir volume of $3 \mathrm{cc}$ and $500 \mathrm{mg}$ of sorbent were obtained from Waters Corporation (Milford, Massachusetts - 01757, USA). HPLC Grade methanol and tetrahydro furan was obtained from Fisher Scientific (Fair Lawn, New Jersey - 07410, USA). Antibody 8E11 prepared from the spleen cells of BPDE-I-G-BSA immunized mice were obtained from Dr.Weston's laboratory.

\section{II.2 Apparatus:}

The liquid chromatograph consisted of a Shimadzu LC10AD pump, Wisp 710B auto-injector (Waters Corporation), an LC-23 column heater (Bio Analytical Systems) set at a temperature of $27^{\circ} \mathrm{C}$, an online Hitachi 1080 fluorescence detector, set at an excitation wavelength of $349 \mathrm{~nm}$ and an emission wavelength 
of 379, and automatic data acquisition through a personal computer using Maxima software (Waters Corporation). SFS reading were by a Perkin Elmer LS50B luminescence spectrometer set at a wavelength difference of $34 \mathrm{~nm}$ and excitation and emission slits of $5 \mathrm{~nm}$ each.

\section{II.3 Human subjects:}

The study was approved by the West Virginia University Institutional Review Board for the Protection of Human Research Subjects. Smoking and nonsmoking paid volunteers were through advertisement and enrolled into the study after written and signed informed consent. Six male and five female smokers were enrolled first and then six male and five female nonsmokers were enrolled after matching for age and weight with the smokers. To be included in the study the smokers were required to be smoking $\geq 20$ cigarettes per day on a regular basis and not smoke cigars or pipes. All nonsmokers were lifelong nonsmokers. Volunteers were instructed to abstain from charbroiled or grilled foods for a week before the day of urine collection. A questionnaire was used to collect data on current smoking habits including brand and average number of cigarettes smoked per day, exposure to secondary cigarette smoke, consumption of alcoholic beverages, medications, caffeinated beverages, char-broiled foods, fresh fruits and vegetables. Volunteers were instructed to maintain a daily diary of all food and drink consumed for a one-week period prior to urine collection.

\section{II.4 Sample Collection and Storage:}

Overnight urine samples were collected at 7:00 a.m in 1.0-liter UrineGuard ${ }^{\circledR}$ containers. Volunteers were instructed to collect any urine void between midnight and 7:00 a.m. in the overnight container. Subsequent urine collections were at 9:00 a.m., 11:00 a.m., 1:00 p.m., 3:00 p.m., 5:00 p.m., 7:00 p.m. and 9:00 p.m. in $250 \mathrm{ml}$ capped, opaque black containers. Volunteers were instructed to void urine only at the specified times as far as possible, but if they felt a necessity 
to urinate between the specified time-points they were instructed to collect those samples in separate containers and note the time of collection. Contents of these were added to the next scheduled collection time of that volunteer in the laboratory. Urine samples were frozen immediately after collection by the volunteer and transported to the laboratory the following morning. The samples were then thawed and the volume in each container was measured and recorded. Urine was aliquoted into $10 \mathrm{ml}$ individually labeled and capped polyethylene tubes and stored in $-80^{\circ} \mathrm{C}$ freezer till analysis.

$10 \mathrm{ml}$ urine samples from ten psoriasis patients undergoing coal-tar treatment (Goeckerman therapy) were obtained frozen from Dr. Weston's laboratory and were stored in $-80^{\circ} \mathrm{C}$ freezer till analysis.

\section{II.5 Analysis:}

$\mathrm{BaPT}$ and 1-OHPG were analyzed in urine involving multiple steps including heating with conc. $\mathrm{HCl}$, extraction using SPE cartridges, immunoaffinity column chromatography, HPLC and SFS.

\section{II.5.1 Heating with concentrated $\mathrm{HCl}$ :}

Tubes containing $10 \mathrm{ml}$ urine aliquot were removed from $-80{ }^{\circ} \mathrm{C}$ refrigerator and allowed to thaw at room temperature. Urine sample was transferred to a glass tube and $80 \mu \mathrm{l}$ of concentrated hydrochloric acid was added to it. The tube was then covered with aluminum foil and heated for 3 hours in a water bath maintained by a thermostat at $90^{\circ} \mathrm{C}$. After removal from the hot water bath the sample was allowed to attain room temperature.

\section{II.5.2 Solid Phase Extraction:}

Extraction of $\mathrm{BaPT}$ and 1-OHPG was done using 3 cc Sep-Pak ${ }^{\circledR}$ solid phase extraction cartridges. In the Varian ${ }^{\circledR}$ sample preparation vacuum manifold the SPE columns were conditioned with $1 \mathrm{ml}$ of methanol followed by $1 \mathrm{ml}$ of water. The sample was then loaded onto the column at a flow rate of about $1 \mathrm{ml} / \mathrm{min}$. After application of the sample, the column was run dry by vacuum and washed 
with $6 \mathrm{ml}$ of water, followed by $2 \mathrm{ml}$ of $30 \%$ methanol in water. BaPT and 1OHPG were eluted with $3 \mathrm{ml}$ of $80 \%$ methanol in water and the eluents collected in labeled glass test tubes. The extracts were then evaporated to dryness in a heated vacuum evaporator.

\section{II.5.3 Immunoaffinity Column Chromatography:}

The residue obtained by drying the SPE extract was reconstituted in $5 \mathrm{ml}$ of 0.1 $\mathrm{M}$ Tris- $\mathrm{HCl}$ buffer at $\mathrm{pH} 7.5$ with the help of ultrasonification. Immunoaffinity columns were conditioned with $5 \mathrm{ml}$ of $0.1 \mathrm{M}$ Tris- $\mathrm{HCl}$ buffer at $\mathrm{pH}$ 7.5. The reconstituted extract was run through the immunoaffinity column at a flow rate of about $1 \mathrm{ml} / \mathrm{min}$. The column was then washed twice with $3 \mathrm{ml}$ of Tris- $\mathrm{HCl}$ buffer at $\mathrm{pH} 7.5$, followed by $3 \mathrm{ml}$ of $30 \%$ methanol in water. Compounds of interest were eluted with $5 \mathrm{ml}$ of $60 \%$ methanol in water. The methanol extract

was then evaporated to dryness under vacuum and then reconstituted in $150 \mu \mathrm{l}$ of $50 \%$ methanol.

\section{II.5.4 High Performance Liquid Chromatography:}

The $150 \mu \mathrm{l}$ extract obtained from immunopurification was injected onto a Phenomenex Prodigy ${ }^{\mathrm{TM}} 5 \mu$ column having length of $100 \mathrm{~mm}$ and internal diameter of $3.2 \mathrm{~mm}$ through a Prodigy ${ }^{\mathrm{TM}} 5 \mu$ guard column having length of 30 $\mathrm{mm}$ and internal diameter $3.2 \mathrm{~mm}$. Both the guard tube and the analytical column were maintained at $27^{\circ} \mathrm{C}$ in an LC-23 column heater (Bio Analytical Systems) throughout the sample run. Mobile phase, $50 \%$ methanol in water, was pumped at a flow rate of $0.5 \mathrm{ml} / \mathrm{min}$. The HPLC fractions were manually collected from the system through retention times 2-4 minutes and 12-14 minutes in separate labeled Eppendorf ${ }^{\circledR}$ tubes. The $1 \mathrm{ml}$ HPLC fractions thus collected were evaporated to dryness and reconstituted in $400 \mu 1$ of water.

\section{II.5.5 Synchronous Fluorescence Spectroscopy:}

SFS readings were taken on the Perkin-Elmer LS 50B luminescence spectrometer using the synchronous mode with a wavelength difference of $34 \mathrm{~nm}$. Both the excitation and emission slits were set at a width of $5 \mathrm{~nm}$. The SFS spectra for both 
the $\mathrm{BaPT}$ and 1-OHPG showed a sharp peak at 346nm, characteristic of the pyrene chromophore.

\section{II.6 Methods development:}

Existing assay procedure for 1-OHPG and BaPT is rather tedious and laborious involving solid phase extraction, immunoaffinity column chromatography, HPLC and SFS. For performing a synchronous fluorescence scan on the HPLC elutes it is necessary to collect the fractions since the SFS cannot be performed online with HPLC. Manual collection of HPLC fractions is very tedious and time intensive. Immunoaffinity columns are prepared from the antibody 8E11 against BaPT prepared from the spleen cells of a BPDE-I-G-BSA immunized mice. ${ }^{70}$ The immunization process extends over a period of several weeks followed by culturing of the spleen cells with hybridoma cells over weeks. This technique is time-consuming and requires skill and experience and may not be performed in many laboratory settings. These antibodies are not commercially available thus limiting the utility of this assay. Hence, experiments were performed to develop an assay for urinary BaPT using solid phase extraction, separation by HPLC and online fluorescence detection of the metabolites. Details of experiments attempting to develop an easy and fast assay without the use of immunoaffinity chromatography are given in Appendix I. Several solid phase extraction cartridges were evaluated for extraction efficiency, but resulted in satisfactory sample clean-up necessary for trace analysis of the metabolites making the use of immunoaffinity column chromatography necessary.

\section{II.6.1 Preparation of Immunoaffinity columns from antibodies against BaPT:}

Monoclonal antibodies against BaPT and 1-OHPG were obtained from Dr. Regina Santella's laboratory through Dr. Ainsley Weston and the immunoaffinity columns were prepared using a protocol developed in their laboratory. A solution of the antibody and protein A sepharose gel was prepared as follows: 
$2 \mathrm{ml}$ of the antibody was centrifuged. The supernatant was removed and diluted with $4 \mathrm{ml}$ of binding buffer (consisting of $3 \%$ PEG 3400, $0.2 \mathrm{M}$ boric acid, $0.2 \mathrm{M}$ triethanolamine) and $0.05 \%$ sodium azide ( $\mathrm{pH} \mathrm{8.9)}$ was added. The solution was filtered through a $0.2 \mu \mathrm{m}$ syringe filter.

$2 \mathrm{ml}$ protein A sepharose 4 fast flow gel slurry was centrifuged at $500 \mathrm{X} \mathrm{g}$ for 1 minute and the supernatant was removed. The gel was resuspended in $5 \mathrm{ml}$ of binding buffer and pelleted again for 1 minute and the supernatant was removed.

Binding the antibody to Protein A gel:

The filtered antibody was added to the gel and the tube was rocked at room temperature for 15 minutes. After pelleting the gel by centrifugation and removing the supernatant, the gel was washed with $5 \mathrm{ml}$ Binding Buffer, pelleted again by centrifugation and the supernatant was removed.

Cross-linking the antibody to Protein A sepharose gel:

Just before use, the dimethyl suberimidate was dissolved in $1 \mathrm{ml}$ Binding Buffer and the solution added to the antibody-loaded Protein A sepharose gel and resuspended. The gel was rocked at room temperature for 15 minutes, collected by centrifugation and the supernatant was removed.

Quenching the crosslinking reaction:

After the cross-linking, the gel pellet was re-suspended in $10 \mathrm{ml}$ of quenching buffer, consisting of $1 \mathrm{M}$ ethanolamine at $\mathrm{pH} 8.5$, and rocked at room temperature for 5 minutes. The gel was re-suspended in $1 \mathrm{ml}$ PBS after centrifugation and removal of supernatant.

Column preparation and packing:

A frit was inserted at the top of the empty column filled with distilled water and seated at the bottom using a hollow tube. The gel slurry was transferred to the column. The gel was packed under a flow of PBS (about 2-4 times the volume of the gel). Once the gel was packed, $1 \mathrm{ml}$ of PBS was added on top of the bed and the top frit was inserted and pushed down slowly so as to sit on top of the gel 
slurry. The column was pre-eluted with $4 \mathrm{ml}$ elution buffer and washed with a few $\mathrm{ml}$ of PBS. The immunoaffinity columns were stored at $4{ }^{\circ} \mathrm{C}$ when not in use.

\section{II.6.2 Extraction efficiency of immunoaffinity columns:}

Four immunoaffinity columns already packed with the antibodies were obtained from Dr. Weston, six more immunoaffinity columns were prepared from antibodies in our laboratory as described above. Since we planned on using all the immunoaffinity columns for analysis it was necessary to determine the extraction efficiency of the columns. In order to be able to use all the columns for analysis all the columns must have have similar extraction.

The extraction efficiency of the columns was determined as follows:

The 10 immunoaffinity columns were conditioned with $5 \mathrm{ml}$ of $0.1 \mathrm{M}$ Tris buffer adjusted to $\mathrm{pH} 7.5$ with hydrochloric acid. $5 \mathrm{ng}$ of $\mathrm{BaPT}$ was loaded onto the column (in $5 \mathrm{ml}$ of Tris- $\mathrm{HCl}$ buffer) at a flow rate of $1 \mathrm{ml} / \mathrm{min}$. The column was then washed twice with $3 \mathrm{ml}$ of Tris- $\mathrm{HCl}$ buffer at $\mathrm{pH} 7.5$, followed by $3 \mathrm{ml}$ of 30 $\%$ methanol in water. Elution was done with $5 \mathrm{ml}$ of $60 \%$ methanol in water. 25

$\mu l$ of each extract and washings were injected into a Phenomenex Prodigy ${ }^{\mathrm{TM}} 5 \mu$ column having length of $100 \mathrm{~mm}$ and internal diameter of $3.2 \mathrm{~mm}$ through a Prodigy $^{\mathrm{TM}} 5 \mu$ guard column having length of $30 \mathrm{~mm}$ and internal diameter 3.2 $\mathrm{mm}$. Mobile phase, $50 \%$ methanol in water, was pumped at a flow rate of 0.5 $\mathrm{ml} / \mathrm{min}$. Extraction efficiency was determined as a percentage of the BaPT extracted using area counts from the HPLC peaks. Analysis of variance (ANOVA) was performed to assess any differences in the columns.

\section{II.6.3 Determination of Retention Time of 1-OHPG :}

$10 \mathrm{ml}$ urine sample obtained from smokers was acidified with $80 \mu \mathrm{l}$ of concentrated hydrochloric acid and subjected to hydrolysis at $90{ }^{\circ} \mathrm{C}$ for 3 hours. The samples were then extracted using Sep-Pak $®$ cartridges as described in Appendix I Exp X. After evaporation and reconstitution in $5 \mathrm{ml}$ Tris buffer the samples were extracted using the immunoaffinity columns as described before. The extract was then evaporated to dryness, reconstituted in $150 \mu \mathrm{l}$ of mobile 
phase and injected onto a Phenomenex Prodigy ${ }^{\mathrm{TM}} 5 \mu$ column (length=100 mm, internal diameter $=3.2 \mathrm{~mm}$ ) through a Prodigy ${ }^{\mathrm{TM}} 5 \mu$ guard column (length $=30$ $\mathrm{mm}$, internal diameter $=3.2 \mathrm{~mm}$ ); the injection volume being $150 \mu \mathrm{l}$. Mobile phase, $50 \%$ methanol in water, was pumped at a flow rate of $0.5 \mathrm{ml} / \mathrm{min}$. The HPLC fractions were collected from the system at intervals of one minute in Eppendorf ${ }^{\circledR}$ tubes and the $0.5 \mathrm{ml}$ HPLC fractions were evaporated to dryness and reconstituted in $400 \mu \mathrm{l}$ of water. Readings were taken on the Perkin Elmer LS 50B luminescence spectrometer set at synchronous mode with a wavelength difference of $34 \mathrm{~nm}$. Three smoker samples were processed as above and the SFS spectra for 1-OHPG (peak maxima at 346 with wavelength difference of $34 \mathrm{~nm}$ ) gave an intense peak in the HPLC elute collected between 2-4 minutes. Strickland et al. have shown that 1-OHPG was the major fluorescent metabolite in immunoaffinity-purified urine and have confirmed the identity of this highly polar metabolite using GC-MS technique. The 1-OHPG is more polar than BaPT which elutes from the HPLC column between 12-14 minutes. Hence for SFS analysis of the 1-OHPG and BaPT the HPLC elutes from 2-4 minutes and 12-14 minutes were manually collected in labeled eppendorf tubes. The $1 \mathrm{ml}$ elute was evaporated to dryness and reconstituted in 400ul of water and SFS readings were taken. 


\section{CHAPTER-III}

\section{RESULTS}

\section{III.1 Comparison of extraction efficiency among columns using ANOVA}

Extraction efficiencies (percentage extraction) obtained from the HPLC peak areas were analyzed by standard ANOVA to assess differences among columns. Percentage extraction from each immunoaffinity column is plotted below.

\section{Extraction Efficiency of Immunoaffinity Columns}

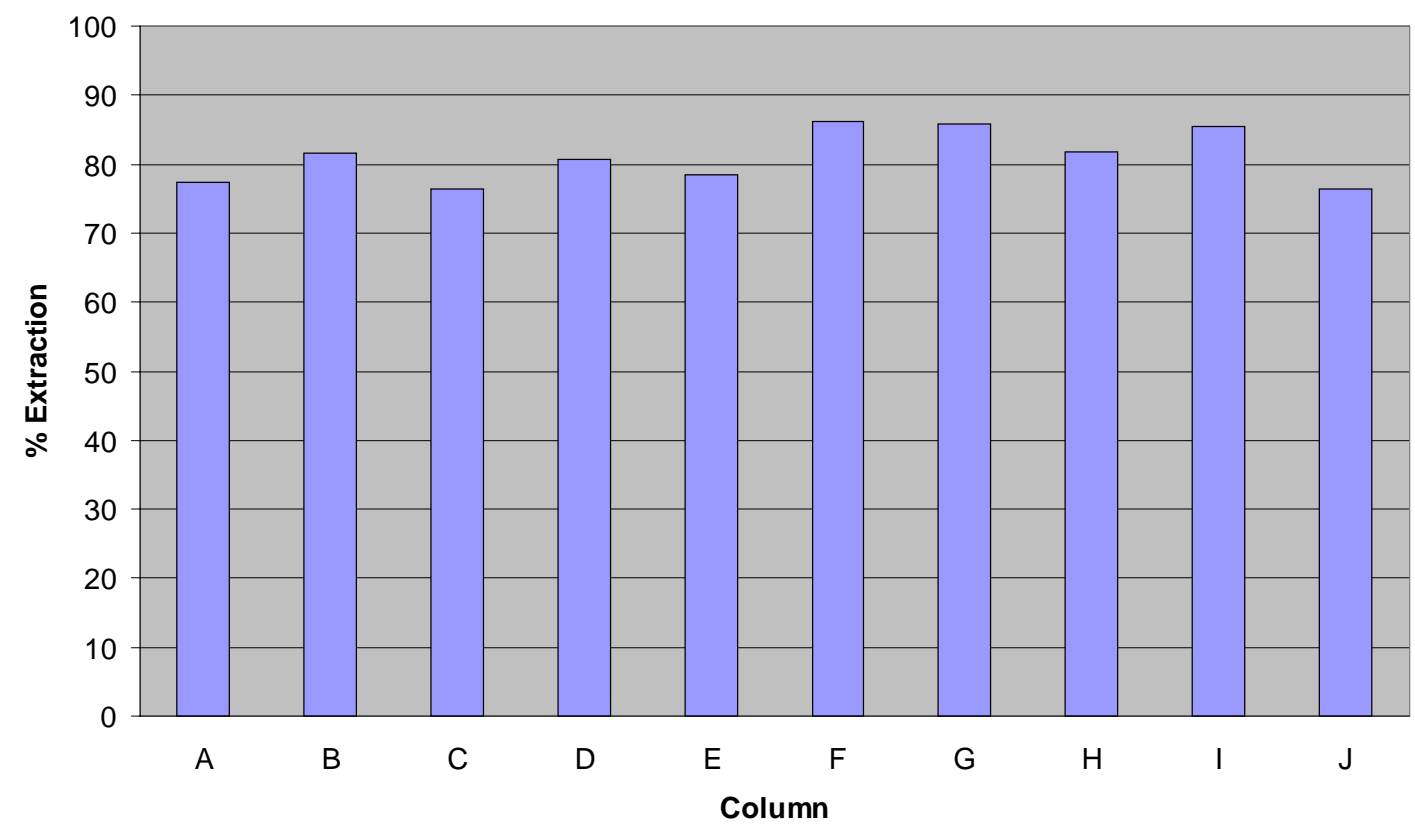

$\mathrm{A}, \mathrm{B}, \mathrm{C}, \mathrm{D}, \mathrm{E}, \mathrm{F}=$ Columns prepared in the laboratory using antibodies against $\mathrm{BaPT}$ $\mathrm{G}, \mathrm{H}, \mathrm{I}, \mathrm{J}=$ Columns obtained from Dr.Weston's laboratory.

\section{Fig.III.1 Extraction efficiency of immunoaffinity columns}

Percentage extraction ranged from $77.3 \%$ to $86.2 \%$, with mean of $80.1 \% \pm 3.58$ in the columns prepared in our laboratory; and from $76.4 \%$ to $85.8 \%$, with mean extraction of $82.35 \% \pm 4.35$ in the pre-filled columns. 


\begin{tabular}{|l|l|l|l|l|}
\hline Group name & Count & Mean & Std. Dev & Std. Err. \\
\hline New column & 6 & $80.10 \%$ & 3.5844 & 1.4633 \\
\hline Old column & 4 & $82.35 \%$ & 4.3555 & 2.1777 \\
\hline
\end{tabular}

ANOVA Results

\begin{tabular}{|l|l|l|l|l|l|}
\hline Source & DF & SS & MS & F & P \\
\hline $\begin{array}{l}\text { Between } \\
\text { Groups }\end{array}$ & 1 & 12.15 & 12.12 & 0.8023 & 0.3966 \\
\hline Within Groups & 8 & 121.15 & 15.14 & & \\
\hline Total & 9 & 133.3 & & & \\
\hline
\end{tabular}

Confidence Level $=95.00 \%$

Critical F $(0.0500,1,8)=5.3177$

No Statistically significant difference was found in the extraction efficiency of the new columns as compared to the old columns $(p=0.3966)$. Overall mean for percentage extraction of $\mathrm{BaPT}$ is $81.0 \% \pm 3.84$ using both pre-filled columns and the new columns. These ten immunoaffinity columns had equivalent extraction efficiencies and thus could be used randomly for sample preparation. Doing so would not add a significant variation due to column difference in the results.

\section{III-2 Description of study population:}

As the paid volunteers were called for by advertisement by banners posted in the university buildings most volunteers were students, faculty and office staff from the university. Male smoker \# 2 was a university police officer and male smoker \# 3 was a painter by occupation. Initially twenty-four volunteers signed up to participate in the study, however two volunteers withdrew from the study due to personal reasons. None of the nonsmokers had smoked cigars, pipes or cigarettes during there entire lifetime. None of the smokers had smoked cigars 
or pipes in the last 6 months and none of them had smoked cigars or pipes on a regular basis. The number of cigarettes smoked per day ranged from 20 to 50 . The number of years they had been smoking cigarettes ranged from 5 to 32 years. Most smokers began smoking during their teens. MS3 did not indicate his age and when began smoking. None of the nonsmokers were exposed to secondary or passive smoking. All male smokers and 2 female smokers indicated exposure to secondary smoke. All male smokers inhaled cigarette puffs deeply while 2 of the female smokers inhaled lightly. The food consumption records maintained by the volunteers confirmed that no PAH-rich foods such as charbroiled meats or smoked foods were consumed by any of the volunteers during the week prior to urine collection. However, they were not in enough detail to assess the intake of pyrene from regular food products.

Smoking history and volunteer data are tabulated (Table III.1). 


\begin{tabular}{|l|l|l|l|l|l|l|l|}
\hline $\begin{array}{l}\text { Volun } \\
\text {-teer \# }\end{array}$ & $\begin{array}{l}\text { Cig/ } \\
\text { day }\end{array}$ & $\begin{array}{l}\text { Years } \\
\text { smok } \\
\text { ed }\end{array}$ & $\begin{array}{l}\text { Age when } \\
\text { began } \\
\text { smoking }\end{array}$ & Inhalation & $\begin{array}{l}\text { Secondary } \\
\text { smoke }\end{array}$ & Weight & Age \\
\hline MS1 & 20 & 9 & 18 & DEEP & Y & 130 & 27 \\
\hline MS2 & 40 & 20 & 15 & DEEP & Y & 172 & 35 \\
\hline MS3 & 30 & 12 & NS & DEEP & Y & NS & NS \\
\hline MS4 & 27 & 5 & 13 & DEEP & Y & 135 & 18 \\
\hline MS5 & 20 & 10 & 20 & DEEP & Y & 175 & 30 \\
\hline MS6 & 50 & 27 & 16 & DEEP & N & 200 & 43 \\
\hline FS1 & 25 & 6 & 14 & DEEP & Y & 120 & 20 \\
\hline FS2 & 20 & 28 & 18 & LIGHT & Y & 165 & 47 \\
\hline FS3 & 20 & 26 & 18 & LIGHT & N & 155 & 46 \\
\hline FS4 & 22 & 32 & 18 & DEEP & N & 185 & 51 \\
\hline FS5 & 25 & 14 & 17 & DEEP & N & 140 & 37 \\
\hline MNS1 & 0 & 0 & NA & NA & N & 190 & 23 \\
\hline MNS2 & 0 & 0 & NA & NA & N & 175 & 31 \\
\hline MNS3 & 0 & 0 & NA & NA & N & 130 & 18 \\
\hline MNS4 & 0 & 0 & NA & NA & N & 155 & 18 \\
\hline MNS5 & 0 & 0 & NA & NA & N & 150 & 45 \\
\hline MNS6 & 0 & 0 & NA & NA & N & 171 & 37 \\
\hline FNS1 & 0 & 0 & NA & NA & N & 125 & 24 \\
\hline FNS2 & 0 & 0 & NA & NA & N & 126 & 45 \\
\hline FNS3 & 0 & 0 & NA & NA & N & 130 & 20 \\
\hline FNS4 & 0 & 0 & NA & NA & N & 140 & 50 \\
\hline FNS5 & 0 & 0 & NA & NA & N & 165 & 49 \\
\hline
\end{tabular}

Table III.1 Volunteer data 


\section{III.3 Synchronous Fluorescence Spectra:}

Synchronous fluorescence spectra for BaPT and 1-OHPG obtained after SPE, immuno-purification and HPLC gave a sharp peak at 346nm (wavelength difference of $34 \mathrm{~nm}$ ) which is characteristic of the pyrene chromophore. Using SFS the limit of detection for BaPT was $25 \mathrm{fmol} / \mathrm{ml}$ given $10 \mathrm{ml}$ of urine sample. SFS is a highly sensitive technique for analysis of BaPT and 1-OHPG and gave much lower detection limits than a post-HPLC column fluorescence detector. SFS is also more selective than regular fluorescence detection and can be used quantitatively.

Representative 1-OHPG and $\mathrm{BaPT}$ spectra obtained from analysis of urine collected from the subjects studied are shown in the following figures. (Fig.III.2 through Fig.III.9). 


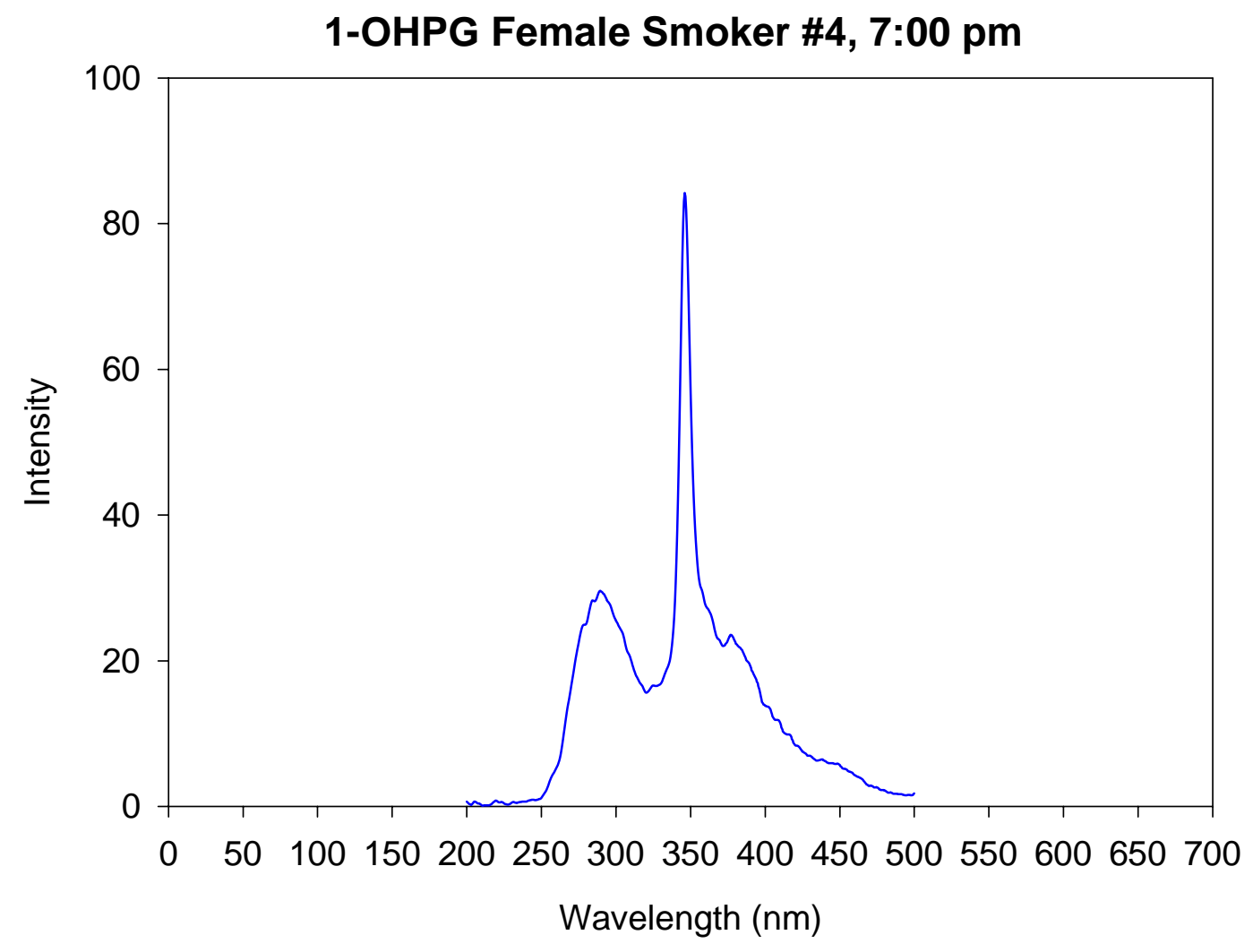

Fig.III.2 Representative 1-OHPG SFS in female smokers 
1-OHPG Female nonsmoker \# 5, 5:00pm

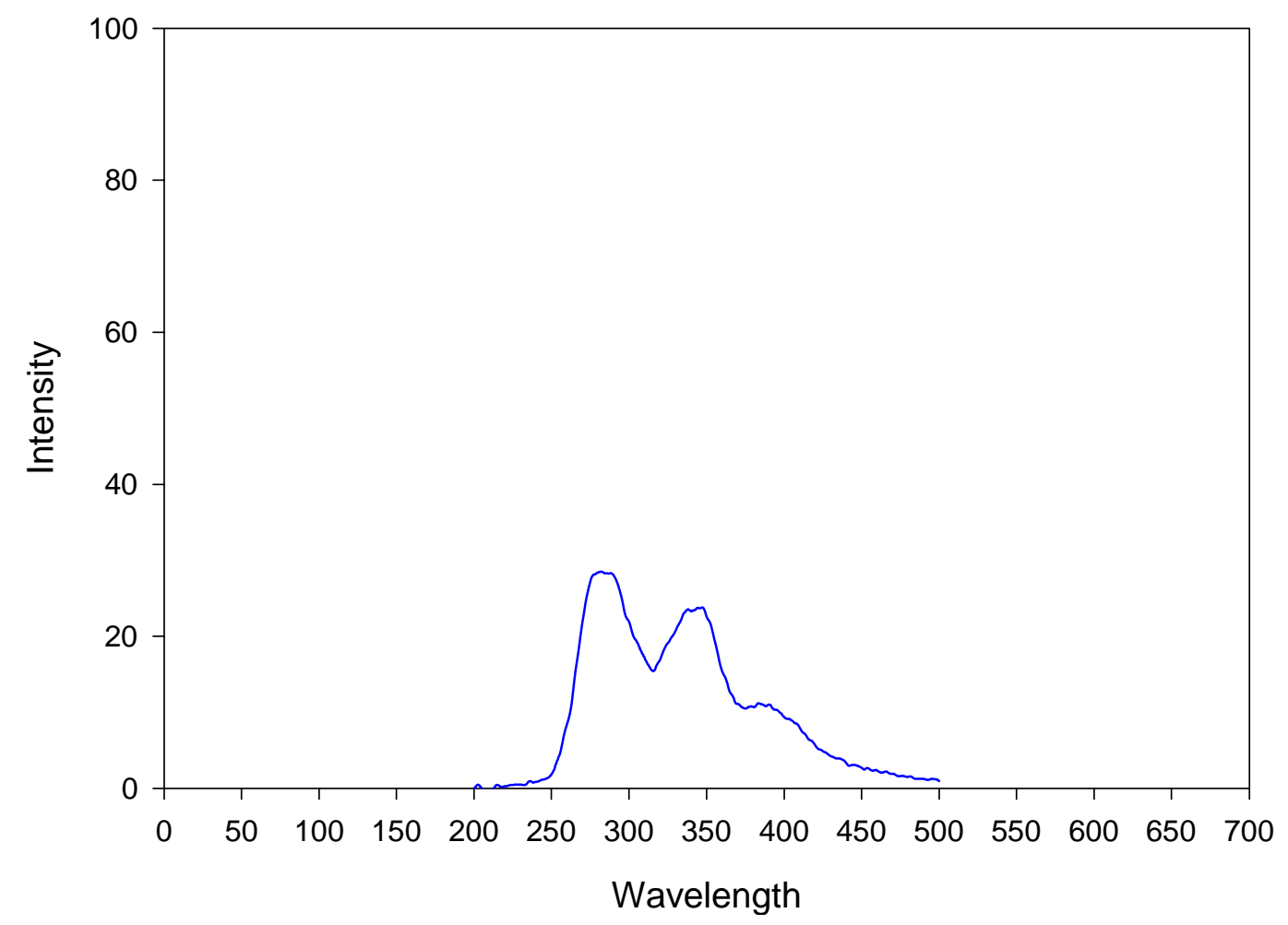

Fig.III.3 Representative 1-OHPG SFS in female nonsmokers 


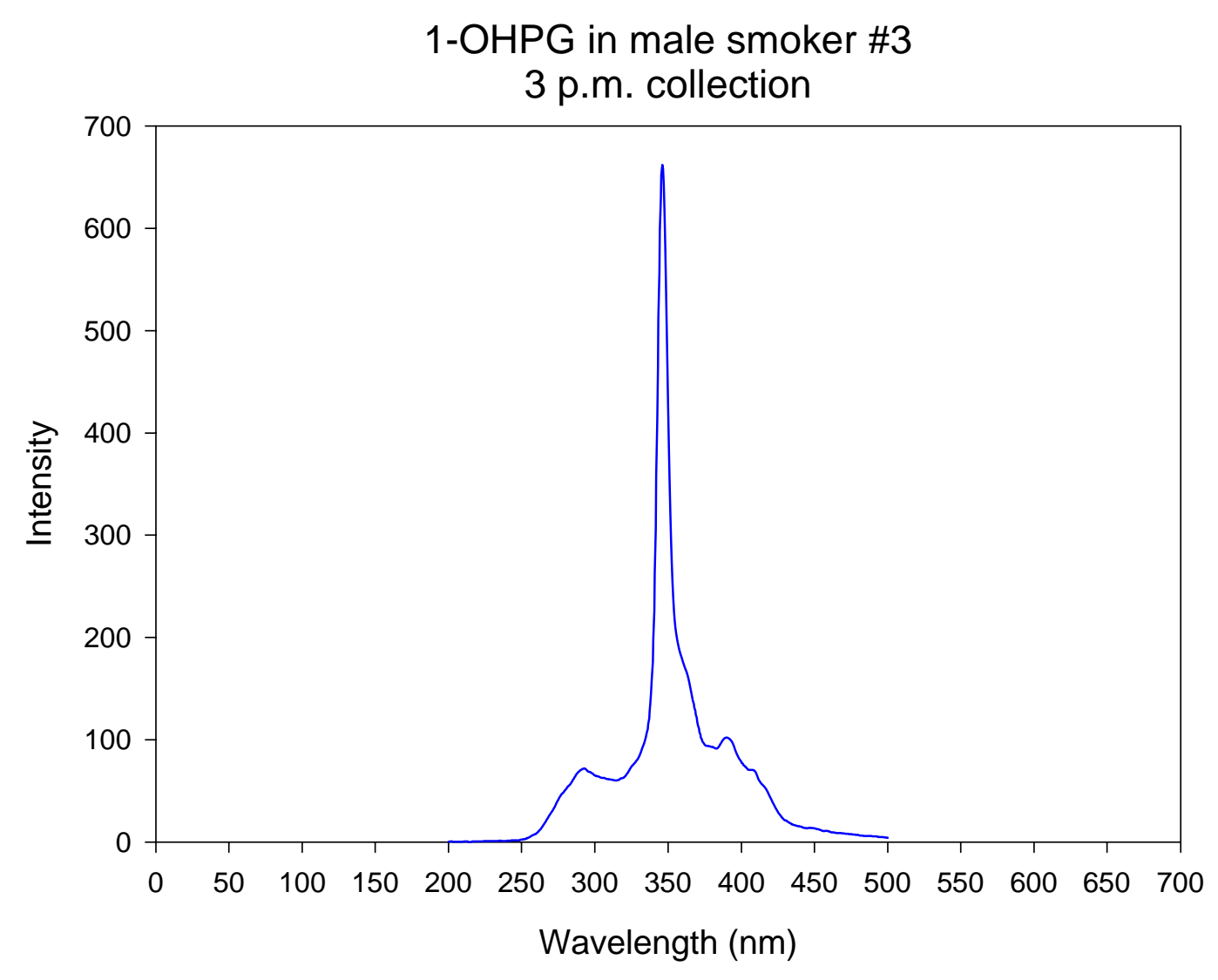

Fig.III.4 Representative 1-OHPG SFS in male smokers 
1-OHPG Male nonsmoker \# 4, 11:00 am

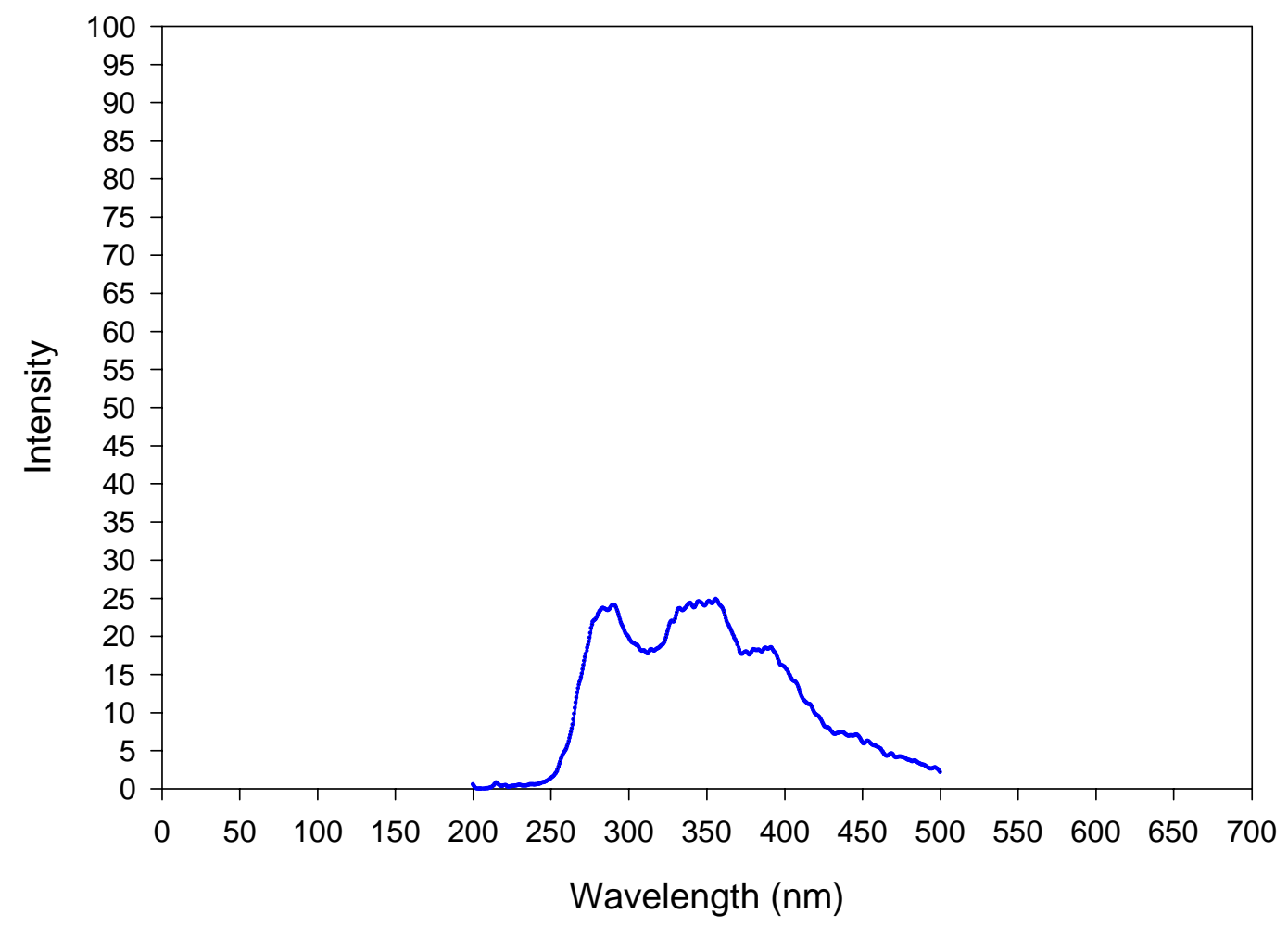

Fig.III.5 Representative 1-OHPG SFS in male nonsmokers 


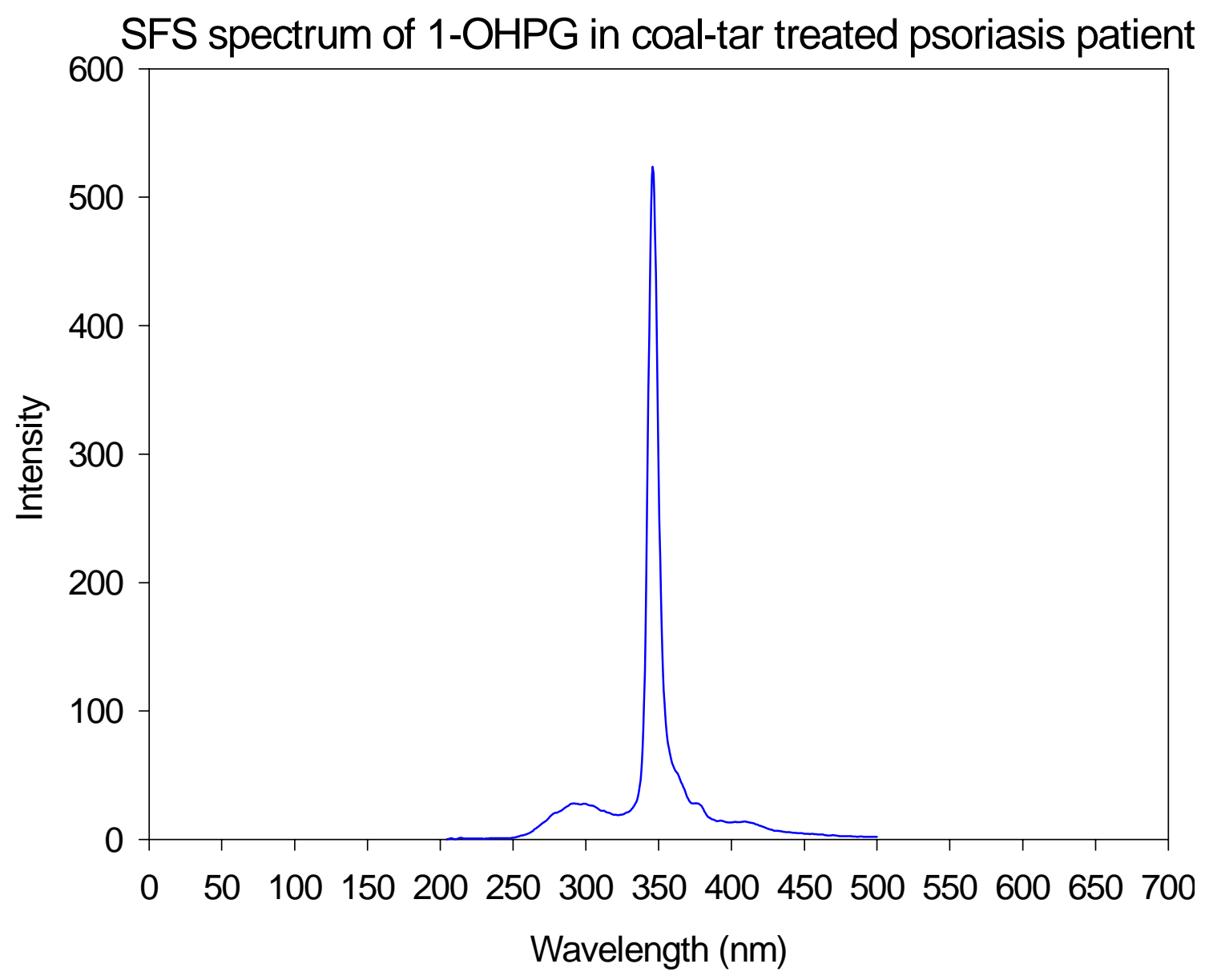

Fig.III.6 Representative 1-OHPG SFS in coal tar treated psoriasis patient 


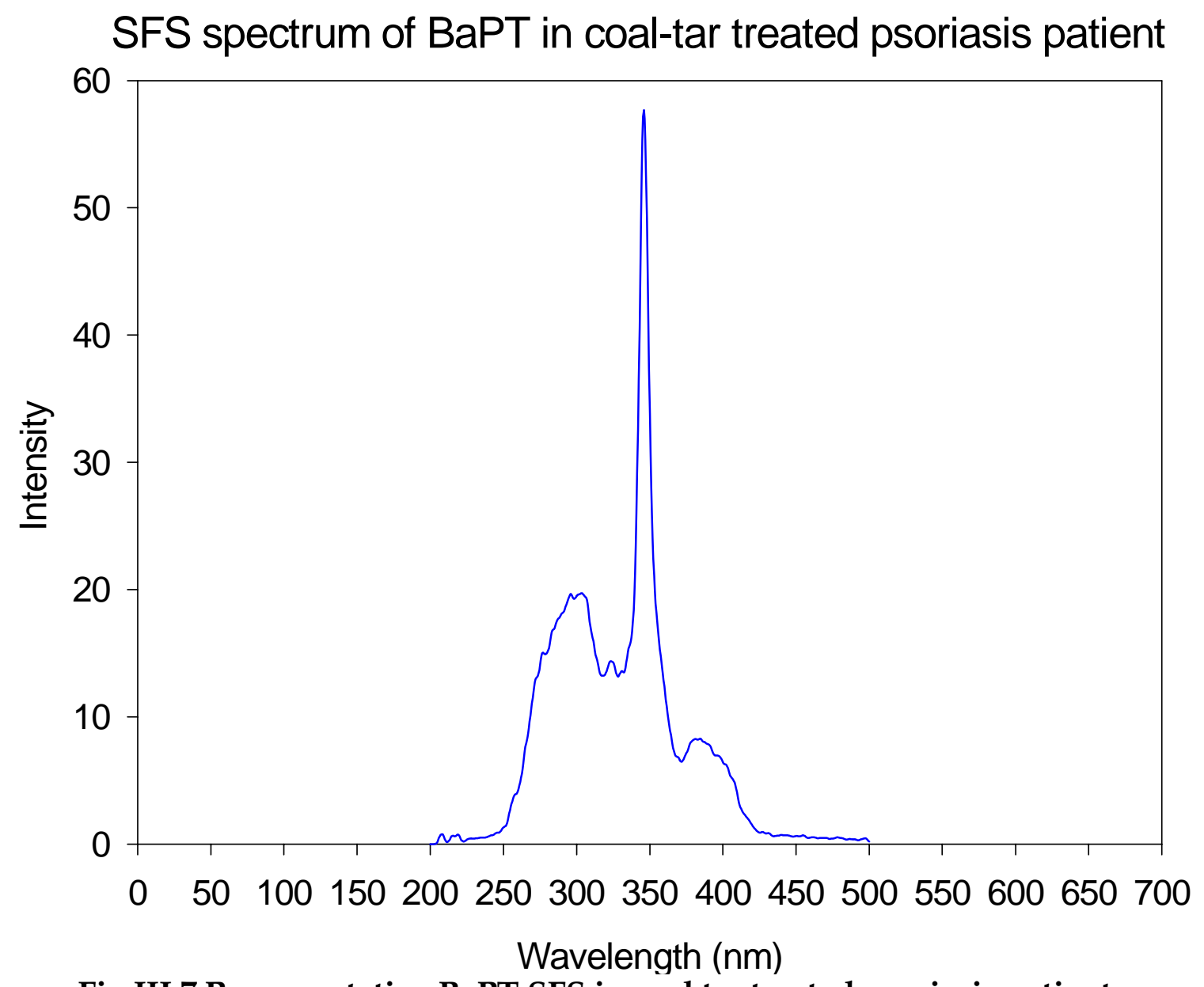

Fig.III.7 Representative BaPT SFS in coal tar treated psoriasis patients 


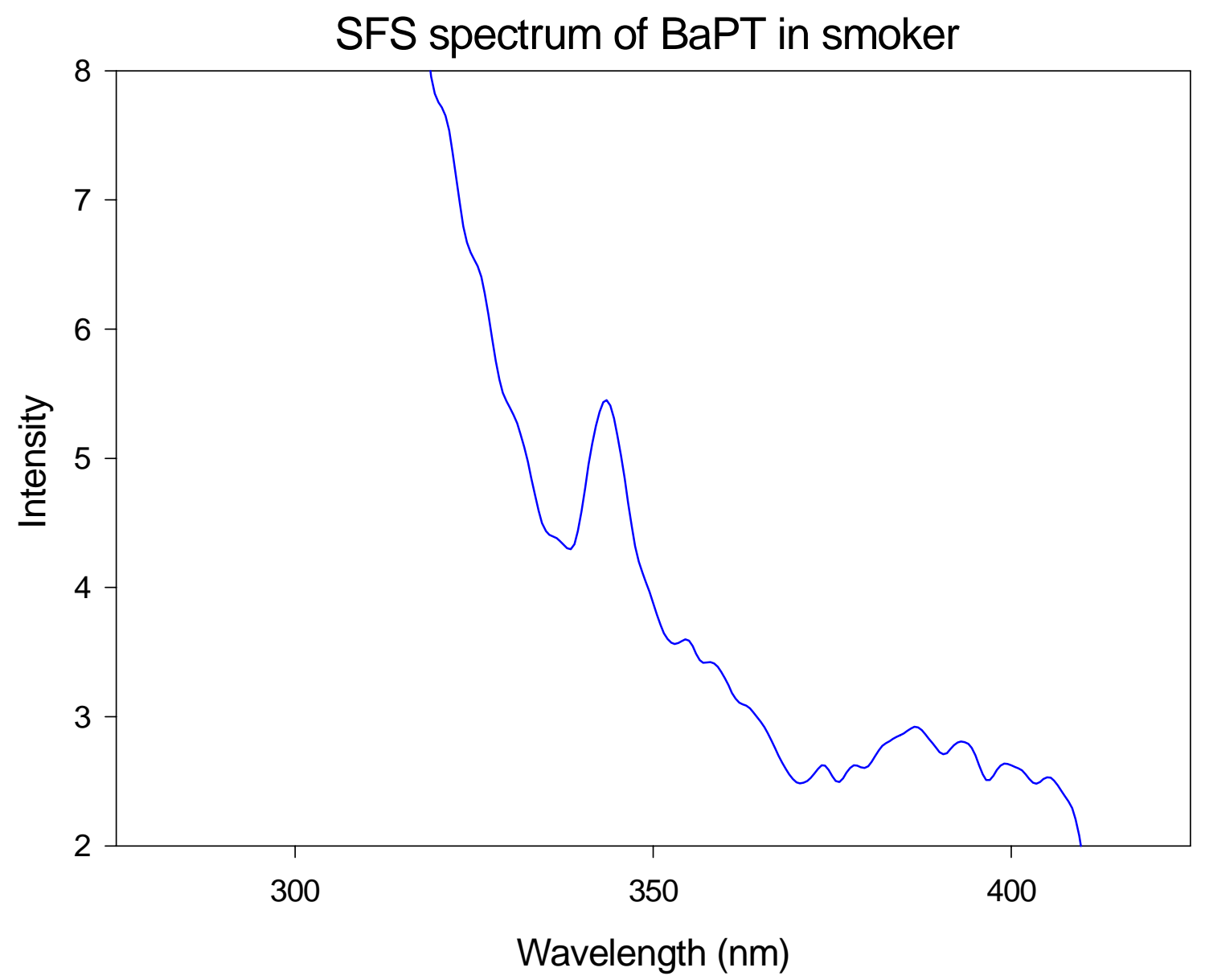

Fig.III.8 Representative BaPT SFS in smokers 


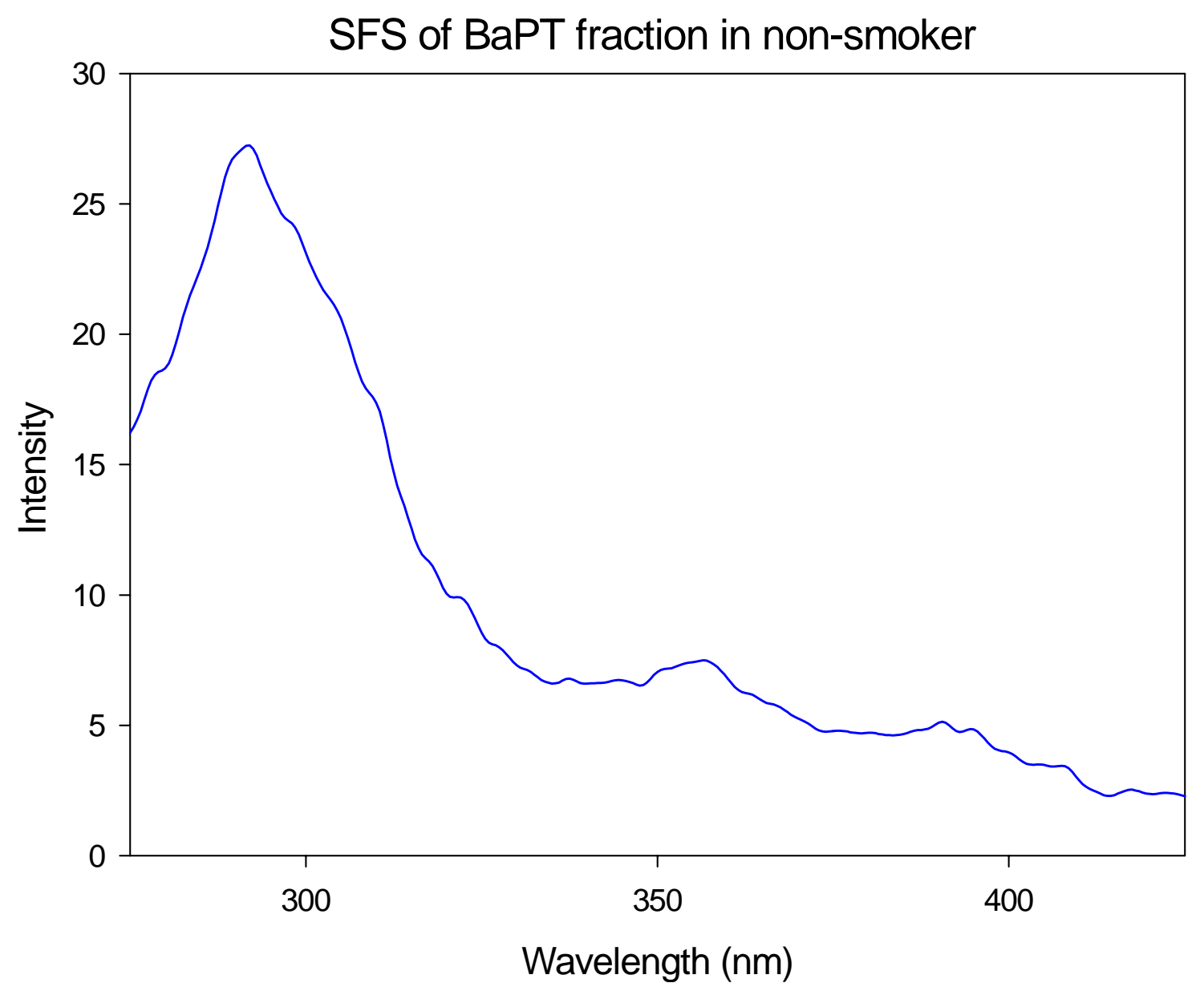

Fig.III.9 Representative BaPT SFS in nonsmokers 


\section{III.3a BaPT calibration curve:}

A calibration curve was generated by spiking nonsmoker urine with known amounts of $\mathrm{BaPT}$ and measuring the SFS peak height after analysis using the procedure described before. Peak height (intensity units) were determined from the SF spectra by drawing a baseline from 342 to $350 \mathrm{~nm}$ and measuring the distance of the perpendicular to the baseline at the point of maximum peak intensity $(346 \mathrm{~nm})$. The limit of detection given $10 \mathrm{ml}$ urine was $2.5 \mathrm{fmol} / \mathrm{ml}$. When SFS peak height (intensity units) was plotted against the BaPT concentration a straight line was generated having the regression equation:

SFS Peak height $=0.0044 *$ BaPT concentration in urine $(\mathrm{fMol} / 10 \mathrm{ml})$.

The coefficient of regression (R-squared) being 0.995, a good linear relationship was seen between BaPT concentration and SFS peak heights (See Fig.III.10).

\section{Standard curve of BaPT in urine}

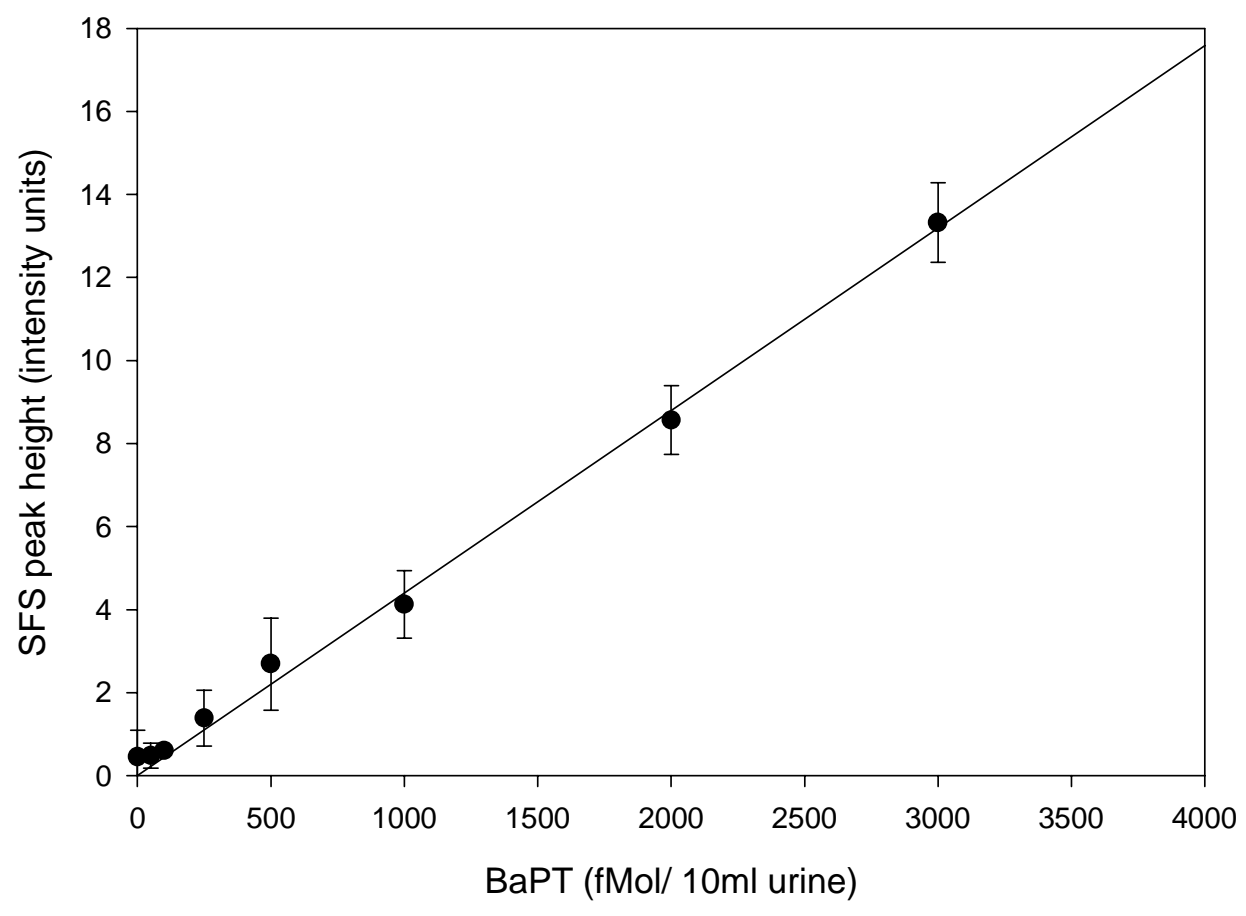

Fig.III.10 Calibration curve for BaPT in urine 


\section{III.4 Analysis of urine samples for BaPT:}

All the 172 samples collected from the smoking and nonsmoking volunteers (6 male and 5 female in each group) were analyzed for BaPT. However, BaPT levels were undetectable in most of the samples. BaPT levels were detected in only 10 of the urine samples (6\% of to samples) all of which were from smokers (8 male and 2 female). Mean BaPT levels in these 10 samples was 230 fmoles/ 10 $\mathrm{ml}$ urine. BaPT levels and corresponding 1-OHPG levels detected are given in the table III.2.

\begin{tabular}{|l|c|c|}
\hline Urine sample & $\begin{array}{l}\text { BaPT } \\
\text { (f mol/10ml urine) }\end{array}$ & $\begin{array}{l}\text { Log (1-OHPG) } \\
\text { (peak height/10ml } \\
\text { urine) }\end{array}$ \\
\hline Male smoker\#1, 7:00 pm & 234 & 1.391464 \\
\hline Male smoker \#3, ovn & 141 & 1.243534 \\
\hline Male smoker \#3, 11:00am & 88 & 1.315551 \\
\hline Male smoker \#3, 3:00pm & 1123 & 2.396705 \\
\hline Male smoker \#3, 7:00pm & 50 & 2.061641 \\
\hline Male smoker \#3, 9:00pm & 95 & 2.302937 \\
\hline Male smoker \#5, ovn & 50 & 0 \\
\hline Male smoker \#5, 9:00am & 290 & 0.933487 \\
\hline Female smoker \#1, 3:00pm & 125 & 0 \\
\hline Female smoker \#3, ovn & 110 & \\
\hline
\end{tabular}

Table III.2 BaPT and 1-OHPG levels from smokers

Linear regression analysis of 1-OHPG with BaPT in the smoker samples was performed by fitting those values to a linear equation using Statmost 3.5 for 
Windows by Dataxiom Software Inc. The R-squared value for the linear trend was 0.222 . The linear equation was:

$\log (1-\mathrm{OHPG})=1.0284+0.0012 * \mathrm{BaPT}$

The linear fit, however, was not statistically significant $(p=0.1695)$. (See figure III.11)

Regression of Log(1-OHPG) with BaPT in samples from smokers

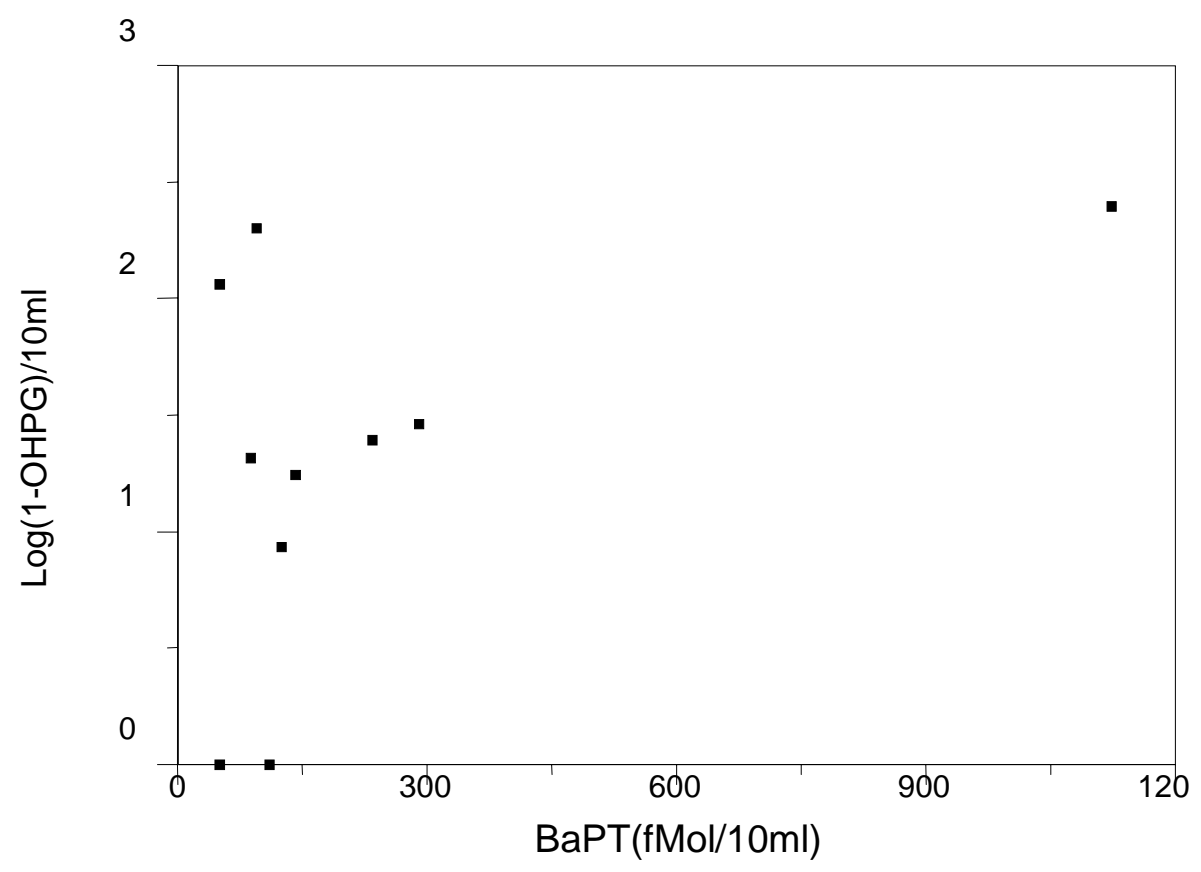

Fig.III.11 Correlation between 1-OHPG and BaPT levels in smokers

The one point with high $\log (1-\mathrm{OHPG})$ value and BaPT levels seemed to unduly influence the fit to show some correlation. Moreover, analysis performed after elimination of that one point resulted in a highly nonsignificant linear relationship $(\mathrm{p}=0.7219)$. 


\section{III.5 Samples from psoriatic patients undergoing coal-tar therapy:}

The $10 \mathrm{ml}$ urine samples obtained from ten psoriatic patients undergoing coal-tar treatment were analyzed for BaPT and 1-OHPG. BaPT was detected in $80 \%$ of the samples (8 out of 10 samples). BaPT levels ranged from undetectable (below $25 \mathrm{fmol} / 10 \mathrm{ml}$ ) to $11 \mathrm{pmol} / 10 \mathrm{ml}$. Mean BaPT in the 10 samples was $4 \pm 3.4$ $\mathrm{pmol} / 10 \mathrm{ml}$.

Most samples from patients undergoing coal-tar therapy had high levels of BaPT in comparison to detectable levels in only few urine samples from smokers. $\mathrm{BaPT} / 10 \mathrm{ml}$ of urine and 1-OHPG SFS peak heights $/ 10 \mathrm{ml}$ urine for the ten samples from psoriatic patients are shown in Table III.3.

\begin{tabular}{|c|c|c|}
\hline Patient \# & $\begin{array}{c}\text { BaPT } \\
(\mathrm{fMol} / 10 \mathrm{ml})\end{array}$ & $\begin{array}{c}1-\text { OHPG } \\
\text { pk height }\end{array}$ \\
\hline 1 & 5134 & 1.17406 \\
\hline 2 & 727 & 2.382737 \\
\hline 3 & 6216 & 1.053078 \\
\hline 4 & 5092 & 2.877314 \\
\hline 5 & $<25$ & 1.301464 \\
\hline 6 & 11285 & 1.731589 \\
\hline 7 & 3658 & 2.389414 \\
\hline 8 & $<25$ & 1.152288 \\
\hline 9 & 2325 & 1.393224 \\
\hline 10 & 5518 & 1.1329 \\
\hline
\end{tabular}

\section{Table III.3 BaPT and 1-OHPG levels from psoriasis patients}

Regression of $\mathrm{BaPT}$ levels and 1-OHPG peak heights in the samples from psoriatic patients failed to show a statistically significant linear relationship ( $\mathrm{P}=$ 0.9308). ( See Fig.III.12). 
Correlation of Log(1-OHPG) with BaPT in Psoriasis Patients

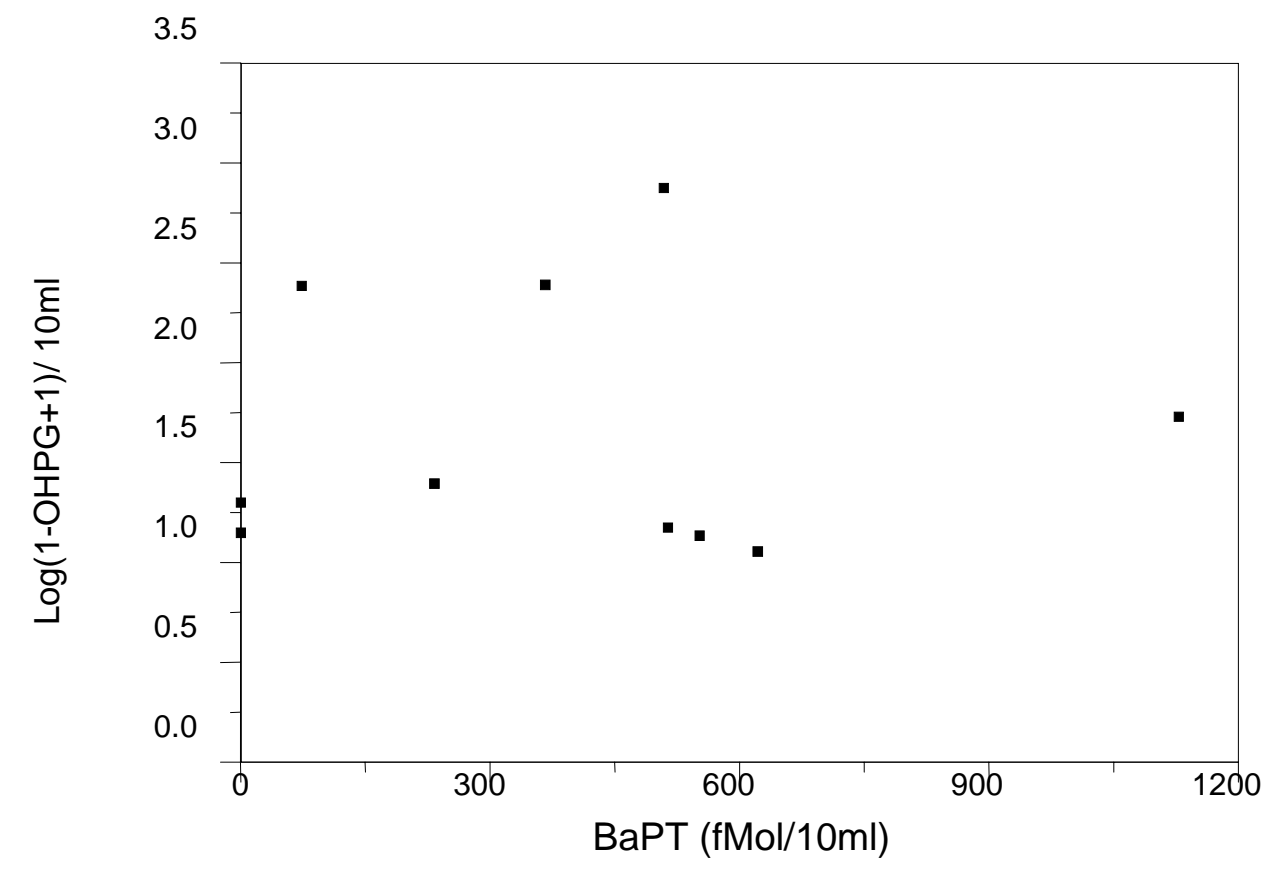

Fig.III.12 Correlation between 1-OHPG and BaPT levels in psoriasis patients

Also, regression of BaPT levels and 1-OHPG peak heights in all the samples in which BaPT was detected (smoker samples + psoriatic patient samples) did not show a statistically significant linear relationship $(P=0.44)$. See Fig.III.13 


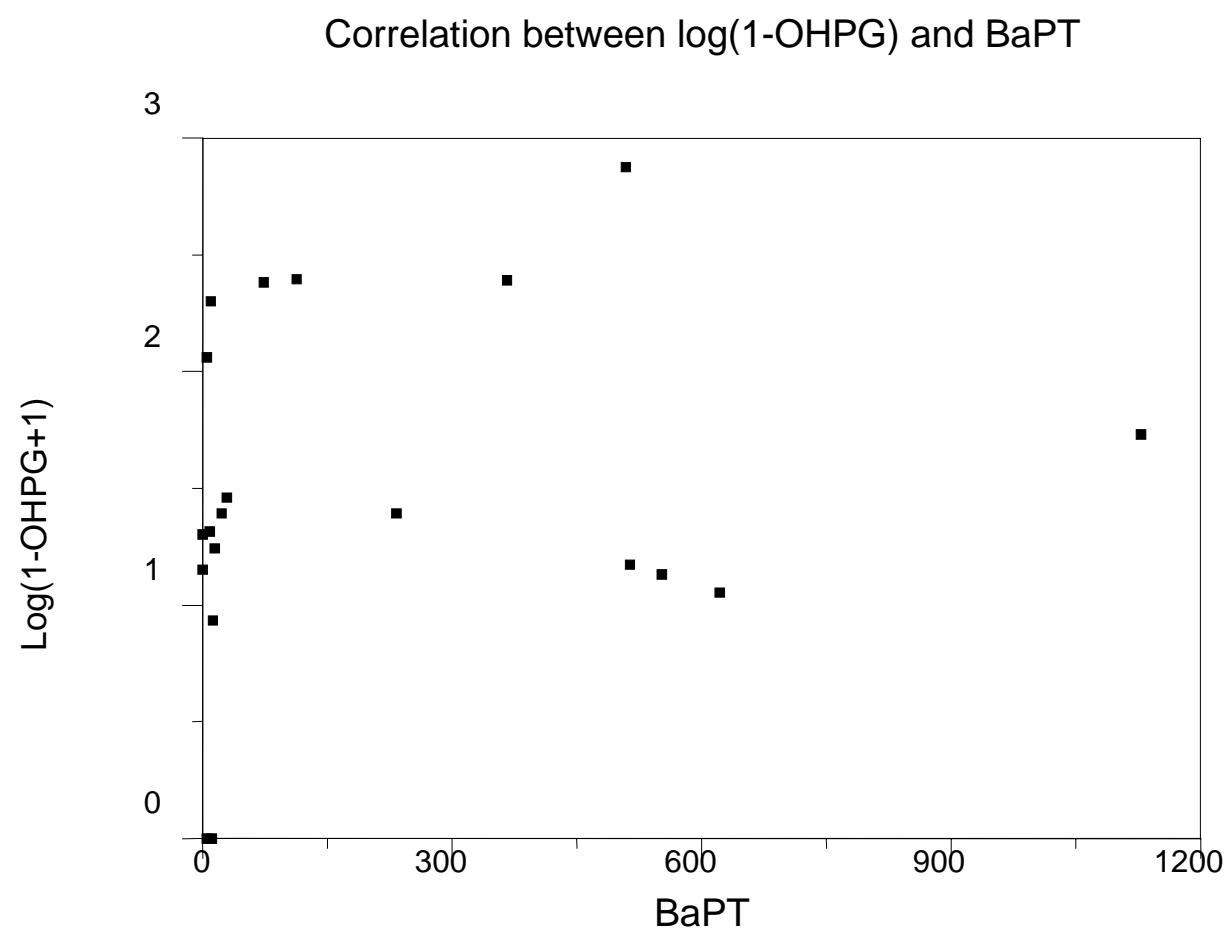

Fig.III.13 Correlation between 1-OHPG and BaPT levels in all samples 


\section{III.6 1-OHPG data:}

1-OHPG was analyzed in all the samples from smokers, nonsmokers and the coal tar treated psoriatic patients as described before and readings were taken by SFS. The SFS peak height for 1-OHPG (intensity units) was multiplied by the urine volume collected after division by 10 (since ten $\mathrm{ml}$ urine was used for analysis) to give the relative amount of 1-OHPG present in that sample.

Authentic standard of 1-OHPG was not available commercially hence a calibration curve for this metabolite could not be generated.

In order to convert the peak height values into concentration terms without having a calibration curve one could attempt a crude estimation by using the calibration curve for BaPT to estimate the 1-OHPG concentration assuming that both have same extinction coefficients. As was necessary, a logarithmic transformation was performed to analyze the data and in any case the means would have to be reported in terms of logarithm of concentration, which has no units of measurement. Also, the assumption that 1-OHPG and BaPT have same extinction is probably not true. Another method of quantification of 1-OHPG without an authentic standard, used by other researchers, is to enzymatically cleave the 1-OHPG to give 1-OHP and estimating the 1-OHPG in terms of the 1OHP standard curve. However, 1-OHPG is about 4 - 5 times more fluorescent than 1-OHP and cleavage of the glucuronide will reduce the assay sensitivity. To avoid these problems this method was not used and the levels of 1-OHPG were used as peak heights (intensity units). The peak height values, in SFS intensity units, obtained as above represent the amount of 1-OHPG in the sample (assuming linearity with concentration in the range studied) and can be treated as apparent 1-OHPG levels as they reflect true 1-OHPG levels. Means obtained after analysis are reported as logarithm of the 1-OHPG levels (no units). 


\section{II.7 Statistical Methods and Analyses for 1-OHPG}

\section{III.7.1 Normalization of data:}

The 1-OHPG peak values in terms of intensity units were calculated from SF spectra as described earlier. The data obtained was badly skewed and the errors were not normally distributed. A logarithmic transformation was performed to normalize the data before statistical analysis as follows:

[ $\log _{10}($ peak height value +1$\left.)\right]$

A constant ' 1 ' was added to the peak height value before taking the logarithm to the base 10 as there were numerous values of 'zero' in the data.

Distribution of errors after the logarithmic transformation was closer to normality as seen in Figure III.14 (Shapiro-Wilk coefficient=0.982247 and mean $=0)$.

\section{Distribution of $E$ hat after transformation}

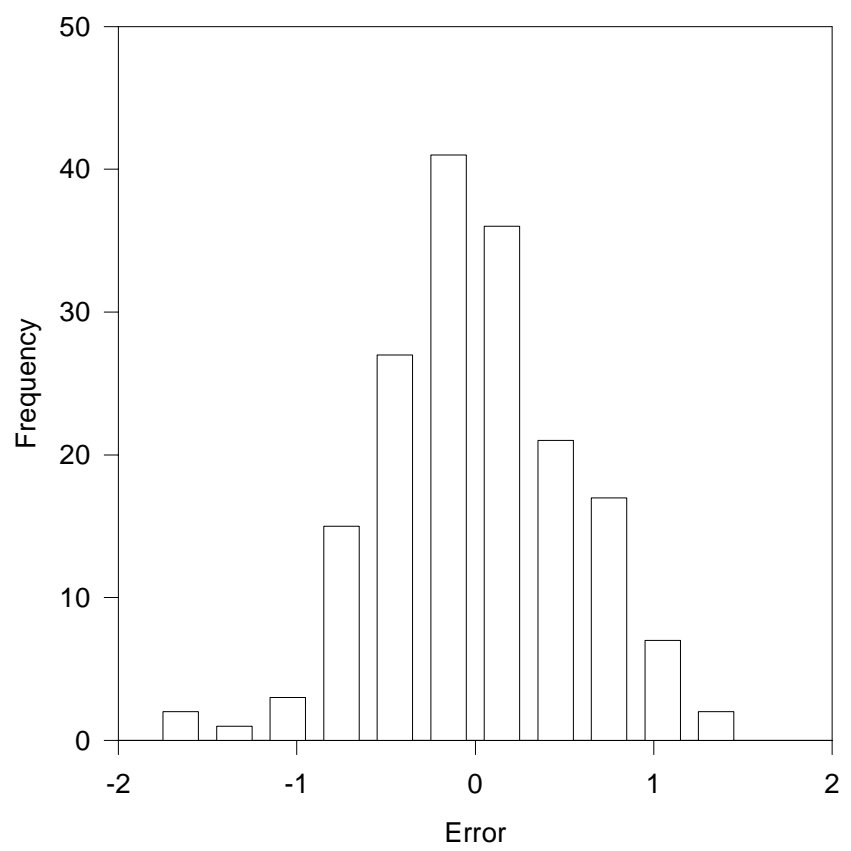

Fig.III.14 Distribution of errors after normalization 
Log-transformed 1-OHPG data was analyzed using analysis of variance using split plot design over time using JMP software by Statistical Analysis Systems Inc. (SAS). Calculations for power, least significant number (LSN), quantile box plots and outlier box plots were created using JMP. Data were examined for possible outliers using the outlier box plot, which is a schematic that allows one to see and identify points with extreme values. Data are expressed as mean $\pm \mathrm{SD}$ except where specified. Differences were considered statistically significant using an alpha level of $0.05(\mathrm{p}<0.05)$.

\section{III.7.2 Distribution of data:}

1-OHPG levels in male smokers, female smokers, male nonsmokers and female nonsmokers at the different collection times are shown in separate plots (Fig. III.15 through Fig.III.18) in order to view the distribution of data. Each volunteer is color symbol coded attempting to show all the points including those where the values are the same. 
1-OHPG levels in Male smokers

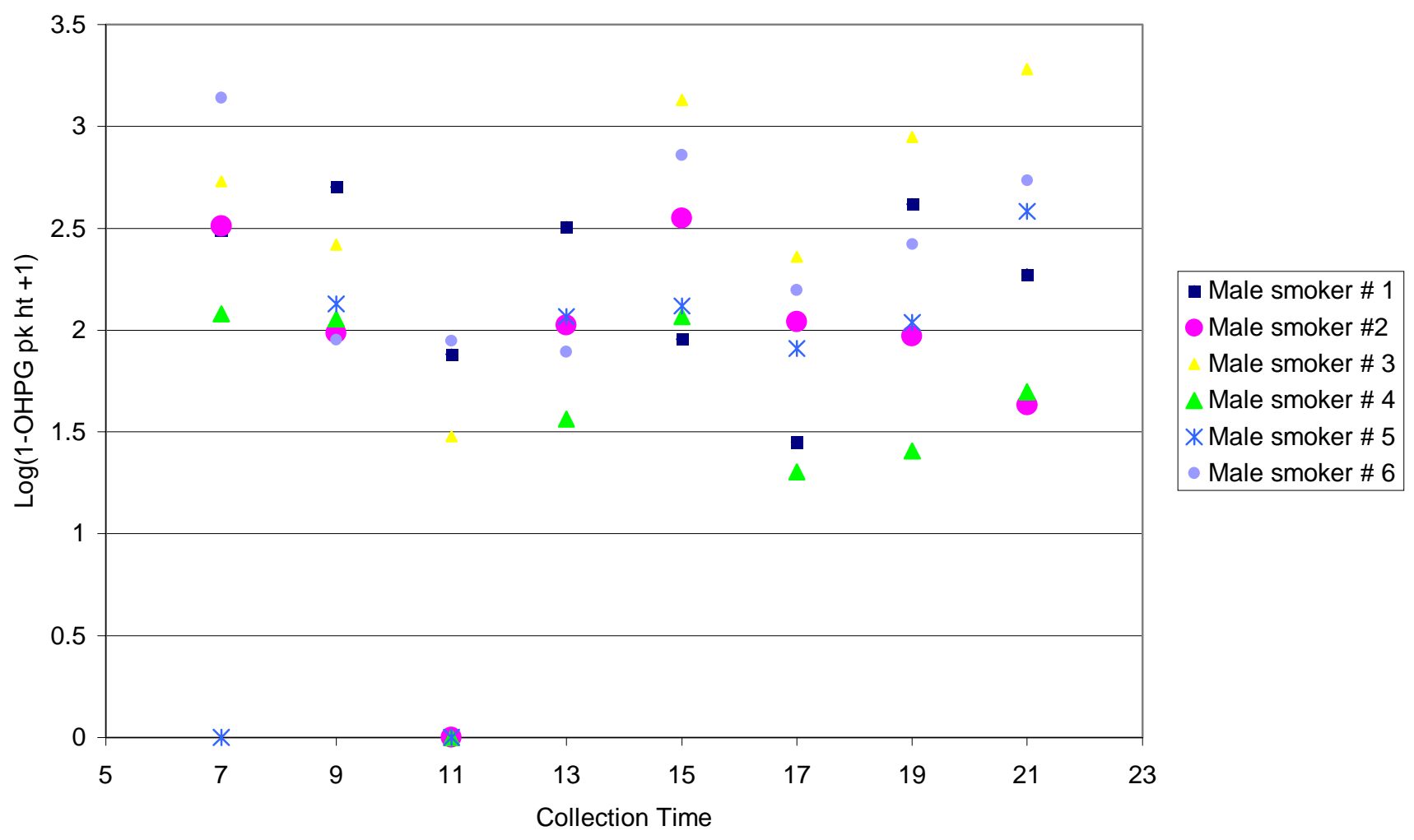

Fig.III.15 1-OHPG levels in male smokers 
1-OHPG levels in Male nonsmokers

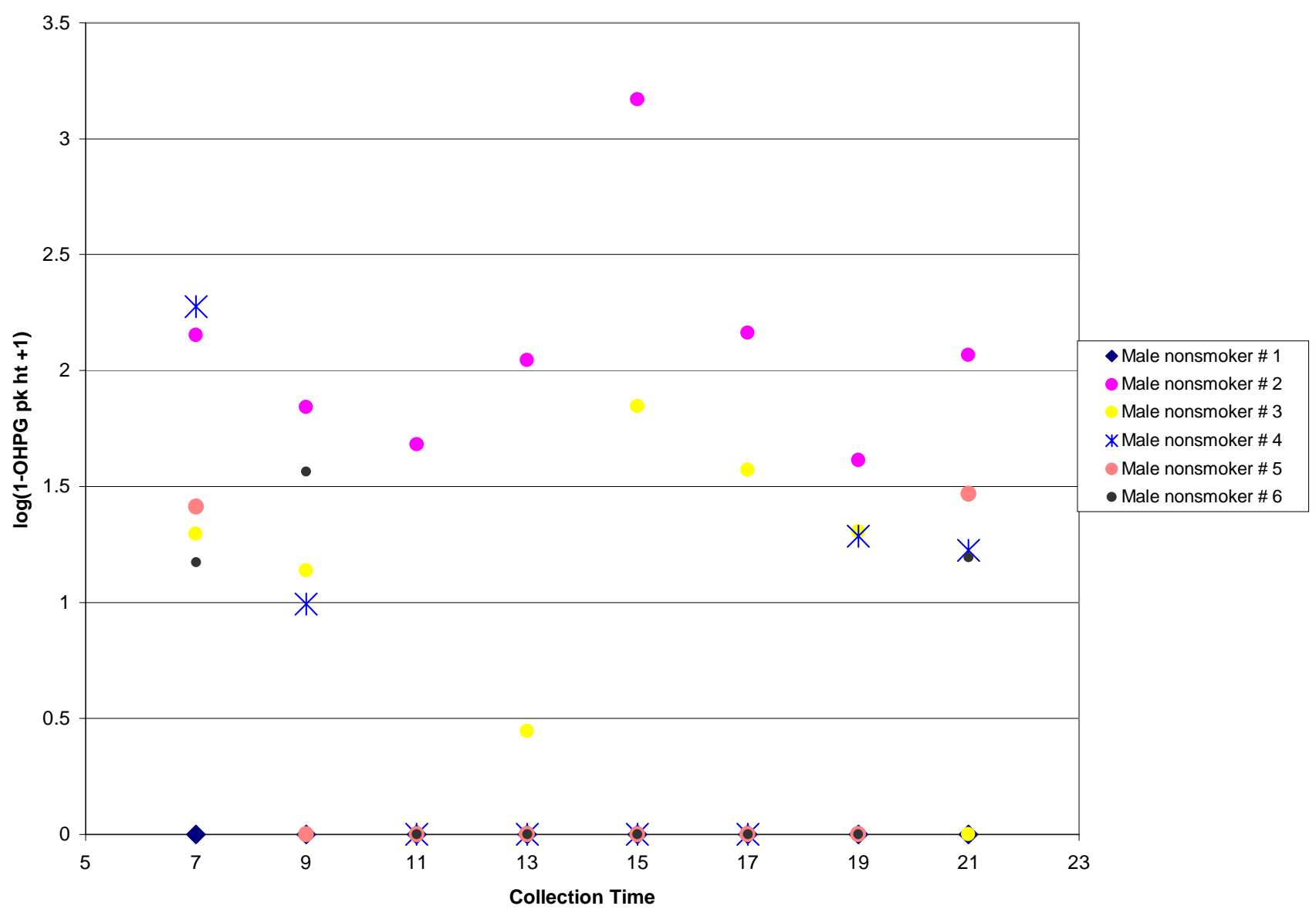

Fig.III.16 1-OHPG levels in male nonsmokers 
1-OHPG in Female Smokers

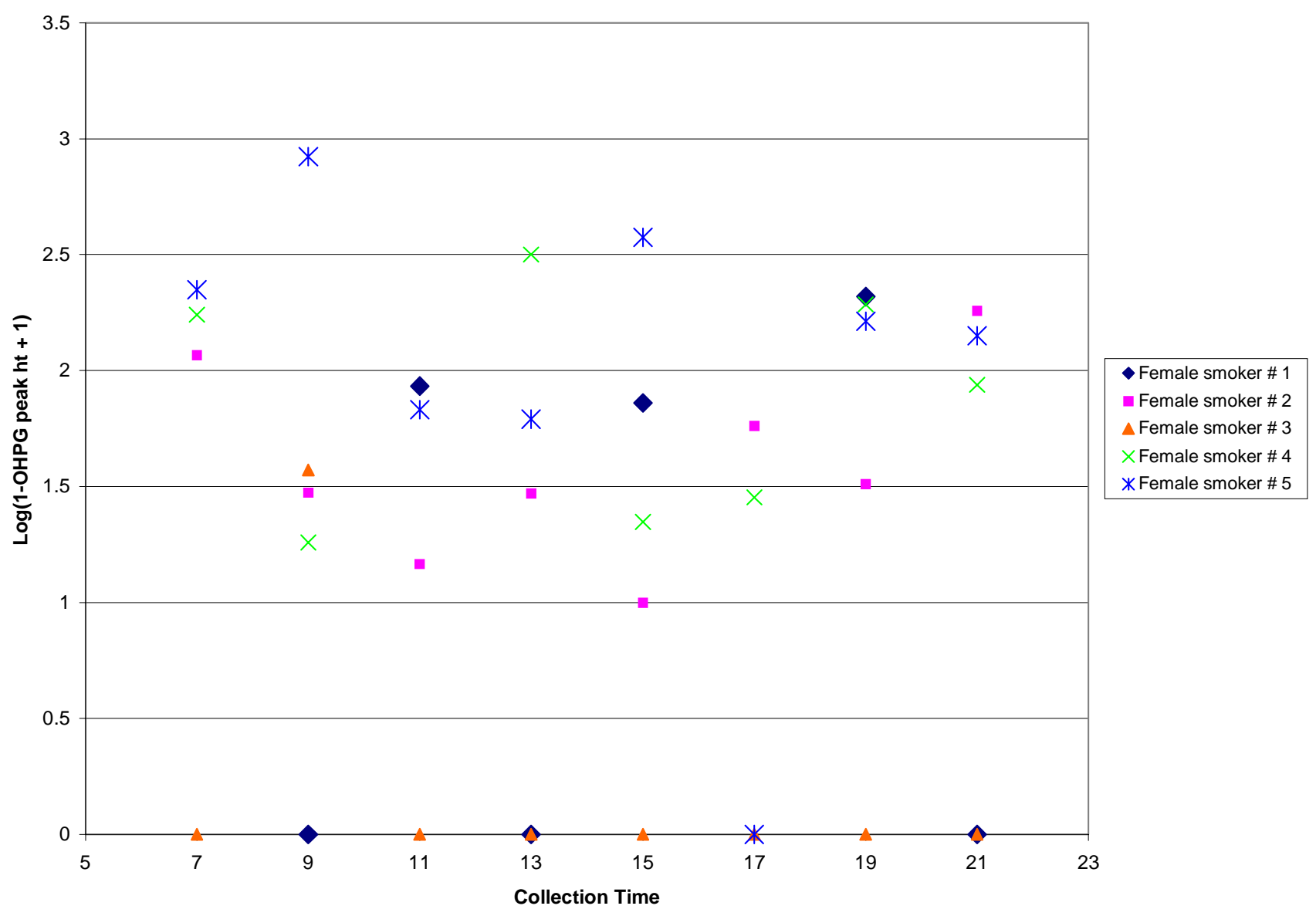

Fig.III.17 1-OHPG levels in female smokers 


\section{1-OHPG in female nonsmokers}

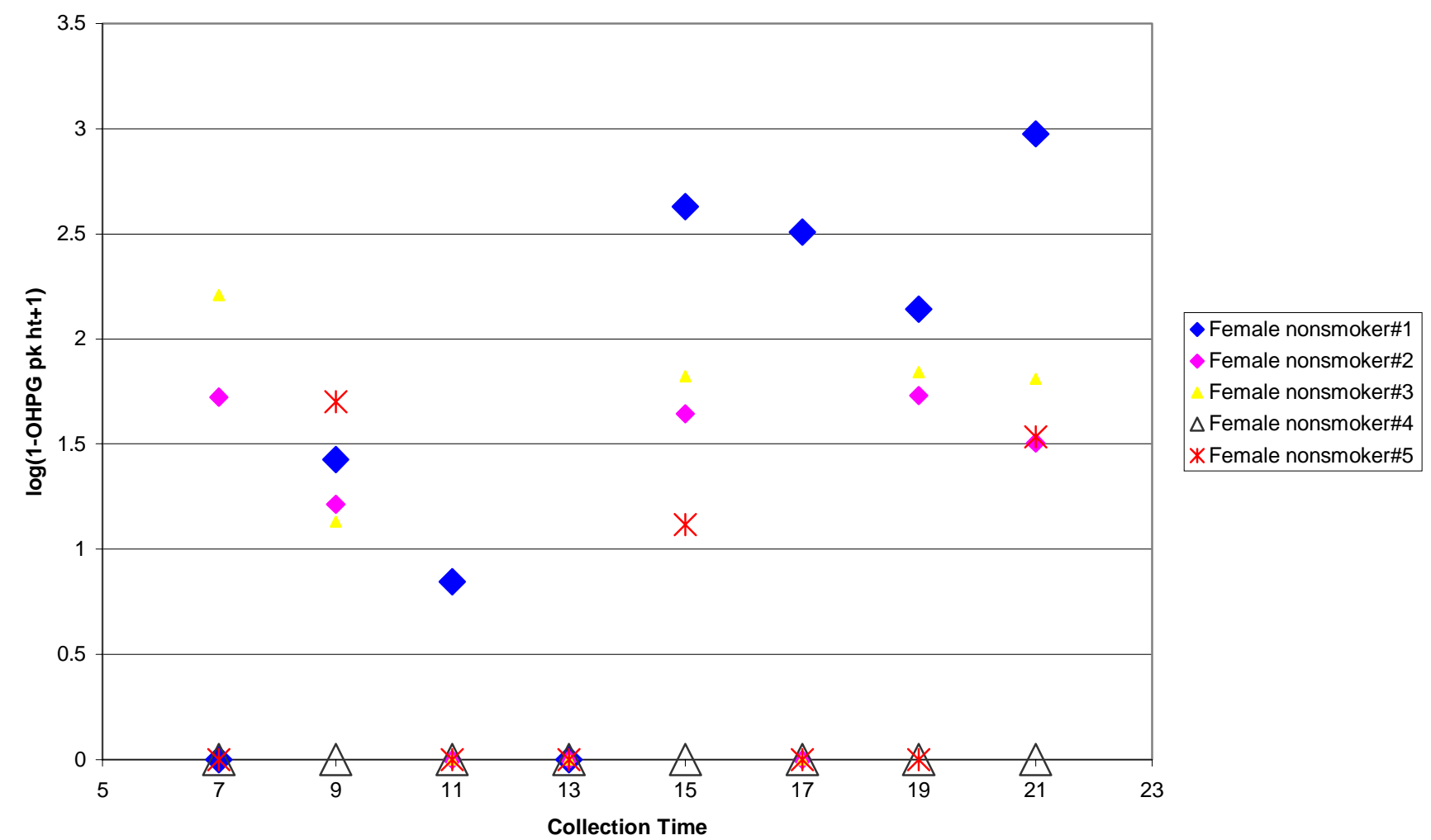

Fig.III.18 1-OHPG levels in female nonsmokers

\section{III.7.3 Testing for outliers :}

Presence of outliers (extreme values) in the data was a concern as such values can bias the data leading to incorrect conclusions. Of most concern was values 
obtained for MS3 which were consistently high at the 3:00 p.m., 5:00 p.m., 7:00 p.m., and 9:00 p.m. collections. In order to compare MS3 with the other subjects in the 'male smokers' group, $\log 10(1-\mathrm{OHPG}$ peak $\mathrm{ht}+1)$ mean over all the collection times for each subject in this group are shown below.

MS1 2.23422

MS2 1.83908

MS3 2.62081

MS4 1.52091

MS5 1.60554

MS6 2.39327

The mean of log10(1-OHPG peak $h t+1)$ for MS3 does not stand out as an outlier or appear to be an extreme high value as compared to the corresponding means of other subjects in the 'male smoker' group.

Quantile box plots are useful to examine the distribution of data. The box shows the median as a line across the middle of the box. The box identifies the $25^{\text {th }}$ and the $75^{\text {th }}$ percentiles (quartiles) as its ends. Whiskers from the top of the box identify the maximum, $99.5^{\text {th }}$ percentile, $97.5^{\text {th }}$ percentile, $90^{\text {th }}$ percentile from top towards the box. Whiskers from the bottom of the box identify the $10^{\text {th }}$ percentile, $2.5^{\text {th }}$ percentile, $0.5^{\text {th }}$ percentile and the minimum. The means diamond identifies the sample mean and the $95 \%$ confidence interval about the mean.

An Outlier Box Plot is a schematic that lets one see the sample distribution and identify points with extreme values, or outliers. The ends of the box are the 25th and 75th quartiles. The red colored bracket along the edge of the box identifies the shortest half, which is the most dense $50 \%$ of the observations. The difference between the quartiles is the interquartile range. The ends of the whiskers are the outer-most data points from their respective quartiles that fall within the distance computed as $1.5^{*}$ (interquartile range). Data points that fall beyond the ends of 
the whiskers are possible outliers. Quantile box plots along with outlier box plots for each group of subjects is shown in figure below. (Fig.III.19).

Male Smokers

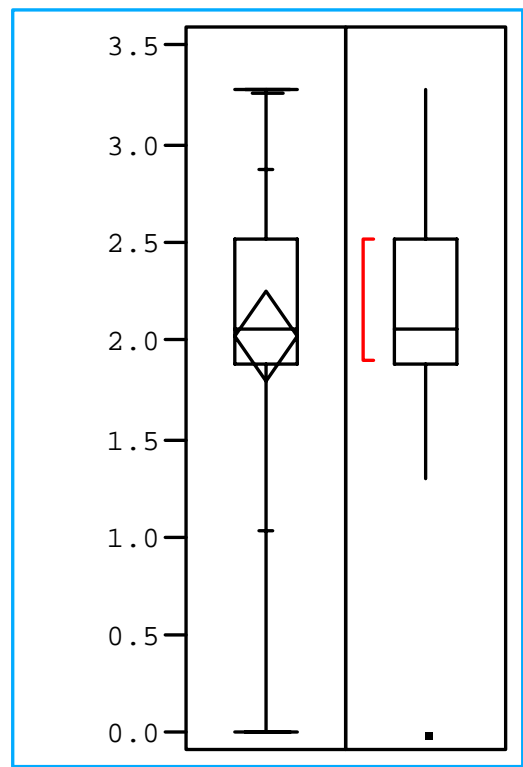

Male nonsmokers

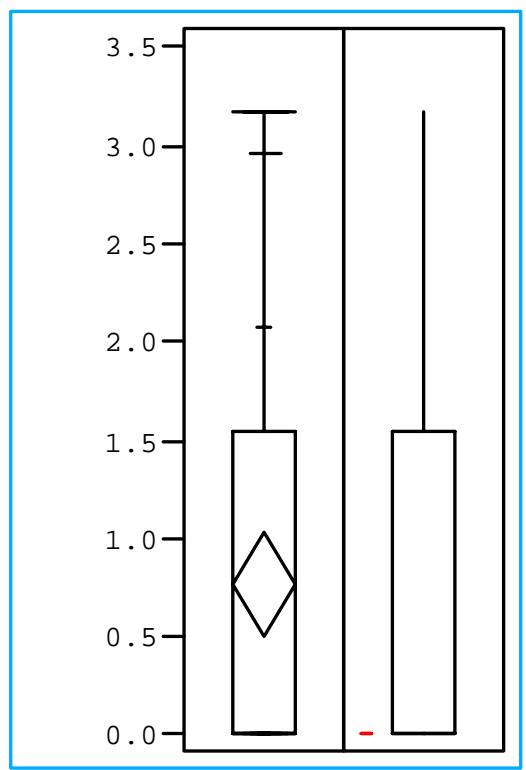

Female Smokers

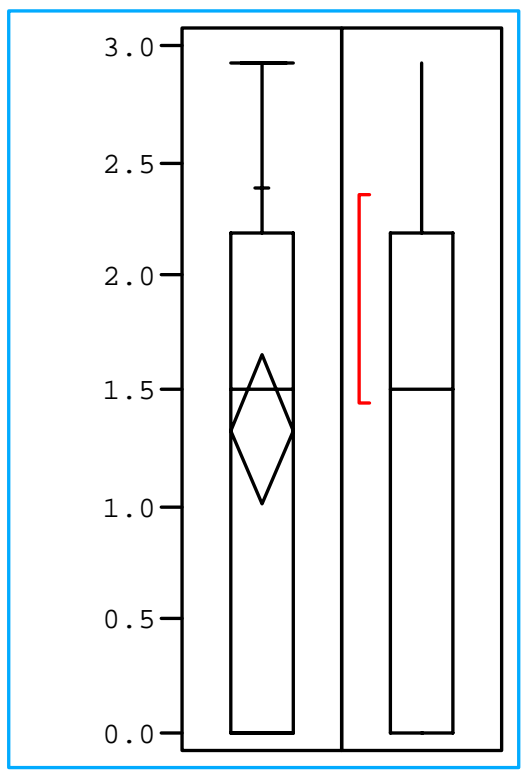

Female nonsmokers

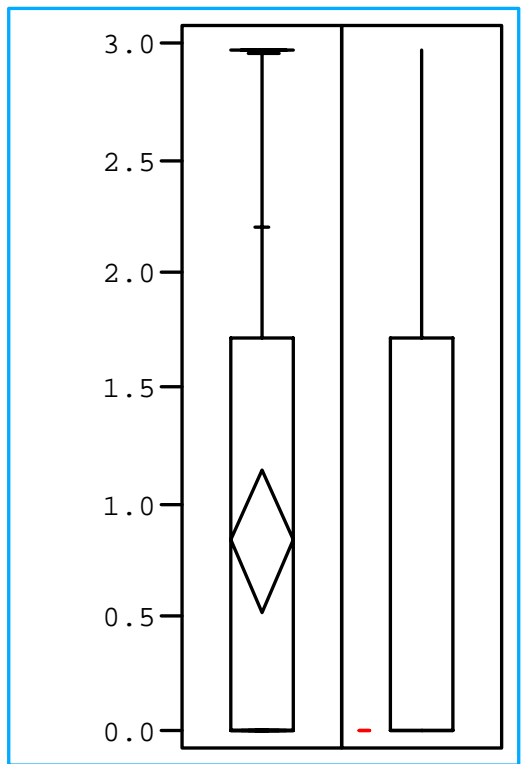

Fig.III.19 Oulier box plots for $\log (1-\mathrm{OHPG}+1)$ data by group 
Median value of $\log (1-\mathrm{OHPG})$ in male smokers and female smokers is 2.06 and 1.51 respectively. While the median in male nonsmokers and female nonsmokers, both are 0.0. Outlier box plots for each group ie. Male smokers, Female smokers, Male nonsmokers, and Female nonsmokers do not have any data point with values higher than the upper whisker suggesting the absence of data points or subjects having extremely high values. Statistical analyses were thus performed taking into consideration all the data points, none being omitted as an outlier.

\section{III.7.4 ANOVA design:}

The experiment was performed on 22 subjects. Subjects were divided into two categories: (smoke) smoker or nonsmoker and (sex) as male or female. Total degrees of freedom (DF) were 171 since total observations were 172 (four missing collection points). Eight measurements were made for 1-OHPG levels for each subject at different collection times. The overall ANOVA design justifying the designation of the error terms to be used for $\mathrm{F}$ value calculations is shown below:

\begin{tabular}{|c|c|c|c|}
\hline & & Smoke & $\mathrm{DF}=(2-1)=1$ \\
\hline \multirow[t]{3}{*}{ Subjects } & \multirow[t]{3}{*}{$\mathrm{DF}=21$} & Sex & $\mathrm{DF}=(2-1)=1$ \\
\hline & & Sex*Smoke & $\mathrm{DF}=1^{*} 1=1$ \\
\hline & & Remainder & $\mathrm{DF}=(21-3)=18$ \\
\hline \multirow{5}{*}{$\begin{array}{l}\text { Measurement } \\
\text { within subjects }\end{array}$} & \multirow{5}{*}{$\mathrm{DF}=(171-21)=150$} & Time & $\mathrm{DF}=(8-1)=7$ \\
\hline & & Sex*Time & $\mathrm{DF}=(1 * 7)=7$ \\
\hline & & Smoke*Tim & $\mathrm{DF}=(1 * 7)=7$ \\
\hline & & Sex*Smoke & Time $\quad \mathrm{DF}=(1 * 1 * 7)=7$ \\
\hline & & Remainder & $\mathrm{DF}=(150-28)=122$ \\
\hline
\end{tabular}

The error term to be used for F-value calculation for Smoke effect, Sex effect and the Sex*Smoke interaction is the Subject(Sex*Smoke) mean square with 18 degrees of freedom. Error term to be used for F-value calculation for Time effect, 
Sex*Time interaction, Smoke*Time interaction and Sex*Smoke*Time interaction is the mean square with 122 degrees of freedom.

\begin{tabular}{lrrrr} 
& \multicolumn{3}{c}{ Analysis of Variance } & \\
Source & DF & Sum of Squares & Mean Square & F Ratio \\
Model & 49 & 131.71534 & 2.68807 & 6.5853 \\
Error & 122 & 49.79953 & 0.40819 & Prob $>$ F \\
C Total & 171 & 181.51487 & & 0.0000
\end{tabular}

Power Details for the Whole Model test

\begin{tabular}{lrrrrrrr} 
Alpha & Sigma & \multicolumn{2}{c}{ Delta Number } & Power & AdjPower & LowerCL UpperCL \\
0.0500 & 0.638900 & 0.875092 & 172 & 1.0000 & 1.0000 & 0.9993 & 1.0000
\end{tabular}

\section{Least Significant Number}

$\begin{array}{rrrr}\text { Alpha } & \text { Sigma } & \text { Delta } & \text { LSN } \\ 0.0500 & 0.638900 & 0.875092 & 62.22236\end{array}$

There was more than enough power in the analysis for the whole model test and it result was highly significant. The least significant number, the minimum number of data points required for a significant result, was about 63. Hence if this experiment were to be repeated again, only 63 points (or 4 subjects in each category) would be enough to reach similar conclusions.

\begin{tabular}{lr}
\multicolumn{1}{c}{ Summary of Fit } \\
RSquare & 0.725645 \\
RSquare Adj & 0.615453 \\
Root Mean Square Error & 0.638900 \\
Mean of Response r & 1.248857 \\
Observations (or Sum Wgts) $\quad 172$
\end{tabular}


Fig.III.20 shows the correlation between the observed $\log (1-\mathrm{OHPG})$ values and the predicted $\log (1-\mathrm{OHPG})$ values as predicted by the model. The R-squared was 0.726 .

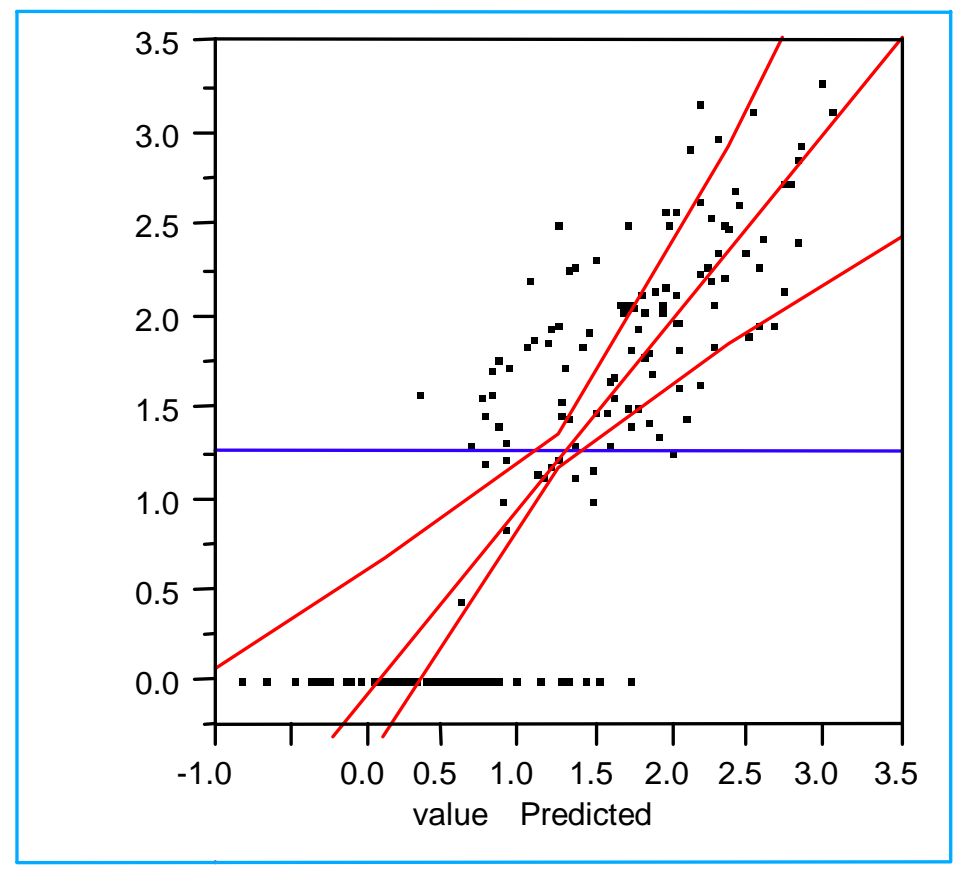

Fig.III.20 Observed 1-OHPG levels versus predicted by the model 
As discussed earlier, for the Smoke effect, Sex effect and Sex*Smoke interaction the correct error term is the Subject[Sex*Smoke] mean squares with 18 degrees of freedom. Significance of these effects can be determined by F-ratios from following table. Statistically significant F-ratios are marked with asterisk. Tests of hypothesis using the Type III MS for Subject[Sex*Smoke] as error

Source

Smoke

Sex

Sex ${ }^{*}$ Smoke

DF Sum of Squares F Ratio Prob $>F$

1

1

1
32.270264

4.247946

6.501268
10.34

1.41

2.07
$0.0048^{*}$

0.2504

0.1678

As was shown in the ANOVA design the following F-ratios are calculated using the mean squares with 122 degrees of freedom as error.

Effect Test (Type III SS)

$\begin{array}{lrrrr}\text { Source } & \text { DF } & \text { Sum of Squares } & \text { F Ratio } & \text { Prob }>\text { F } \\ \text { Time } & 7 & 16.498960 & 5.7742 & 0.0000^{*} \\ \text { Sex*Time } & 7 & 3.197541 & 1.1191 & 0.3555 \\ \text { Smoke*Time } & 7 & 2.512878 & 0.8794 & 0.5249 \\ \text { Sex*Smoke*Time } & 7 & 6.014488 & 2.1049 & 0.0479 * \\ \text { Subject[Sex,Smoke] } & 18 & 56.140784 & 7.6408 & 0.0000^{*}\end{array}$


Power calculations for the smoke effect are shown below.

Power Details for Smoke effect

$\begin{array}{lrrrrrrr}\text { Alpha } & \text { Sigma } & \text { Delta Number } & \text { Power } & \text { AdjPower } & \text { LowerCL UpperCL } \\ 0.0500 & 0.638900 & 0.433149 & 172 & 1.0000 & 1.0000 & 1.0000 & 1.0000\end{array}$

\section{Least Significant Number}

$\begin{array}{rrrr}\text { Alpha } & \text { Sigma } & \text { Delta } & \text { LSN } \\ 0.0500 & 0.638900 & 0.433149 & 51.77237\end{array}$

The least significant number for smoke effect was about 52. This suggests that if the study were to repeated then significant difference would be seen between smokers and nonsmokers even if only 52 data points were collected (about 3 smokers and 3 nonsmokers).

In order to illustrate the difference in 1-OHPG levels between smokers and nonsmokers quantile box plots showing the distributions of data for each of the groups are shown below (Fig.III.21). 
SMOKERS

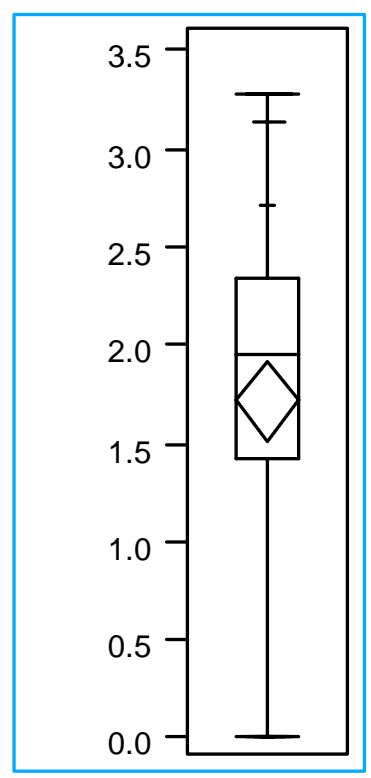

NONSMOKERS

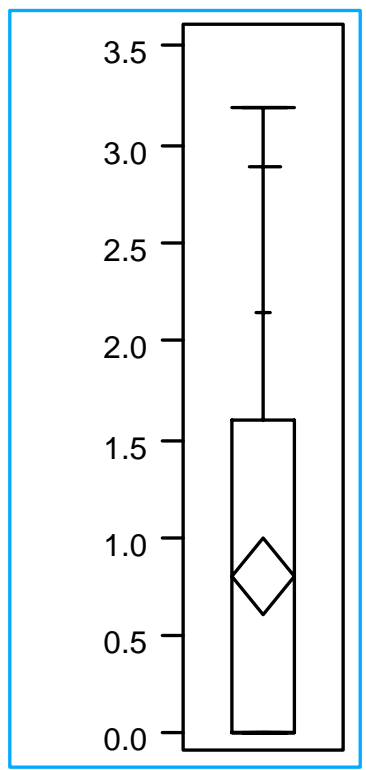

Fig.III.21 Quantile box plots and mean in smokers and nonsmokers

\begin{tabular}{|lcc|}
\hline & $\begin{array}{c}\text { Smokers } \\
\text { Quantiles }\end{array}$ & \\
Maximum & $100.0 \%$ & 3.2815 \\
& $99.5 \%$ & 3.2815 \\
& $97.5 \%$ & 3.1381 \\
quartile & $90.0 \%$ & 2.7163 \\
median & $75.0 \%$ & 2.3419 \\
quartile & $50.0 \%$ & 1.9536 \\
& $25.0 \%$ & 1.4166 \\
& $10.0 \%$ & 0.0000 \\
& $2.5 \%$ & 0.0000 \\
minimum & $0.5 \%$ & 0.0000 \\
& $0.0 \%$ & 0.0000 \\
Mean & Moments & \\
Std Dev & 1.71843 & \\
Std Err Mean & 0.92558 & \\
\end{tabular}

\begin{tabular}{|lcc|}
\hline & $\begin{array}{l}\text { Nonsmokers } \\
\text { Quantiles }\end{array}$ \\
& $100.0 \%$ & 3.1702 \\
& $99.5 \%$ & 3.1702 \\
Maximum & $97.5 \%$ & 2.8972 \\
quartile & $90.0 \%$ & 2.1431 \\
median & $75.0 \%$ & 1.6036 \\
quartile & $50.0 \%$ & 0.0000 \\
& $25.0 \%$ & 0.0000 \\
& $10.0 \%$ & 0.0000 \\
minimum & $2.5 \%$ & 0.0000 \\
& $0.5 \%$ & 0.0000 \\
Mean & $0.0 \%$ & 0.0000 \\
Std Dev & & \\
Std Err Mean & 0.009062 & \\
& 0.09845 & \\
\hline
\end{tabular}


Cigarette smoking had a statistically significant effect on the levels of 1-OHPG $(p=0.0048) . \log 10(1-\mathrm{OHPG}+1)$ values are higher in smokers $(1.71 \pm 0.9256)$ as compared to nonsmokers $(0.80 \pm 0.9235)$. Mean of $\log 10(1-\mathrm{OHPG}+1)$ levels being about 2-fold higher in smokers in comparison to nonsmokers. Median value for smokers was 1.95 in comparison to a median of 0 for nonsmokers. More than $50 \%$ of the nonsmoker samples had undetectable levels of 1-OHPG in comparison to only $10 \%$ of the smoker samples. About $75 \%$ of the samples from smokers had $\log 10(1-\mathrm{OHPG}+1)$ values greater than 1.5 while about $75 \%$ of the samples from nonsmokers had values lesser than 1.5. The value of 1.5 may be used as a cut-off point to discriminate between smokers and nonsmokers.

Sex effect (whether male or female) on the levels of 1-OHPG was not statistically significant $(\mathrm{p}<0.2504)$. Male subjects had a mean value of $1.39 \pm$ 1.05 compared to $1.08 \pm 0.99$ for female subjects.

The interaction between Sex effect and Smoke effect also did not reach statistical significance $(\mathrm{p}<0.1678)$. A plot of the means for each group indicates that urinary 1-OHPG levels in male smokers seem to be higher compared to those in female smokers, while levels in male nonsmokers and female nonsmokers were about the same. As neither the sex effect nor the interaction was statistically significant a comparison of levels between male smokers and female smokers was not attempted in order to protect the overall alpha level. A Plot of means in each group is shown below (Fig III.22). 


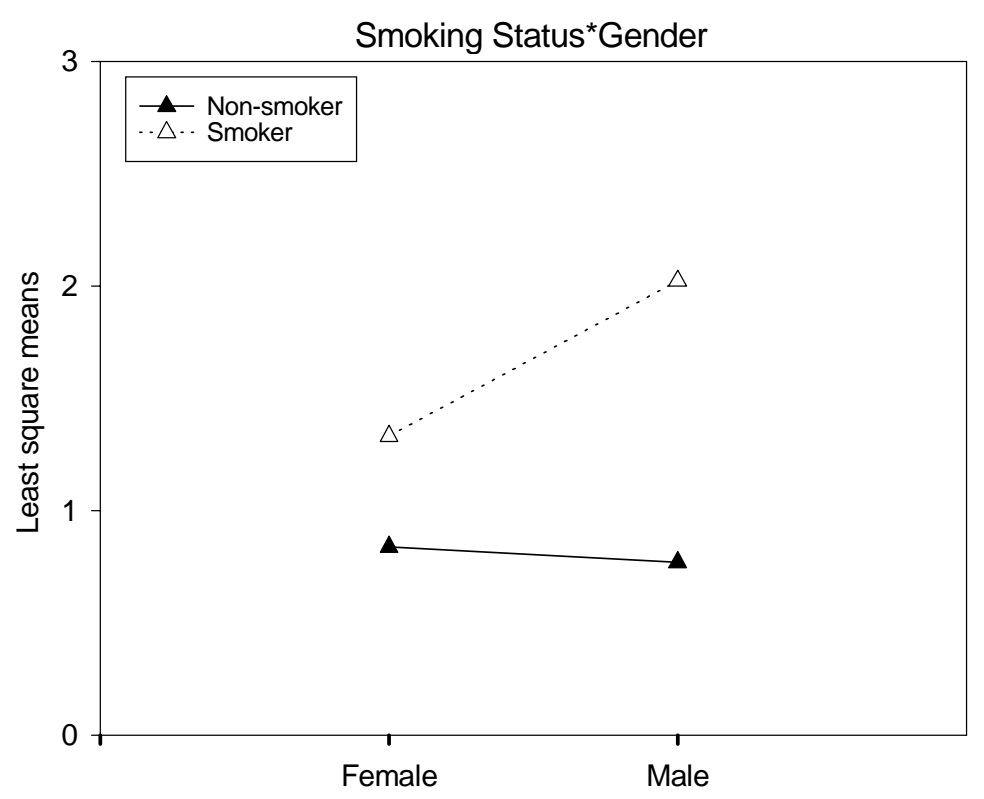

Fig.III.22 Smoking status and gender interaction

Although sex differences are discussed one should remember that neither the sex effect nor the sex*smoke interaction were statistically significant.

1-OHPG levels at the various collection times were significantly different $(p=0.00)$ and the Sex*Smoke*Time interaction was significant $(p=0.0479)$ which suggests that the smoke effect was different for males and females at the various collection times. Hence each collection time was investigated for sex effect, smoke effect and the sex ${ }^{*}$ smoke interaction.

ANOVA model used for this analysis was as follows:

There were 22 subjects at each time point contributing to a total $\mathrm{DF}=(22-1)=21$ at collection times with no missing values.

Smoke $\quad \mathrm{DF}=1$

Sex $\quad D F=1$

Smoke*Sex $\quad \mathrm{DF}=1$ 
Error $\quad \mathrm{DF}=18$

Mean square from the error term was used to calculate the F-ratio.

A plot of the mean 1-OHPG values in smokers and nonsmokers is shown below (Fig.III23) to illustrate the differences. The p-values associated with the difference between smokers and nonsmokers at each collection time are shown on the graph above each time point.

Differences in 1-OHPG levels between smokers and nonsmokers with each collection time

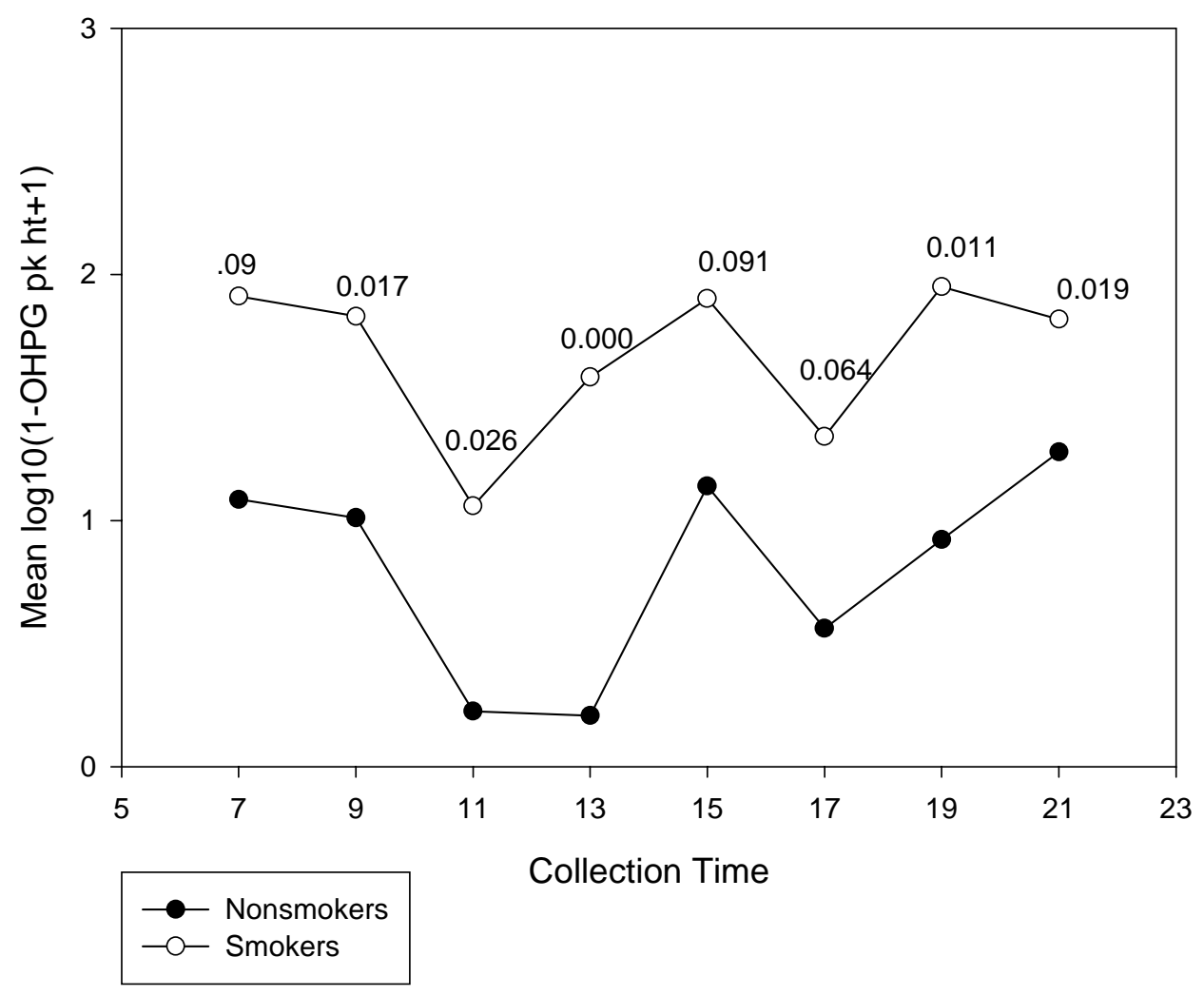

Fig.III.23 Mean 1-OHPG levels in smokers and nonsmokers with time 
1-OHPG levels were higher in smokers in comparison to nonsmokers at all the collection times. The difference was statistically significant at the 9:00 a.m., 11:00 a.m., 1:00 p.m., 7:00 p.m., and 9:00 p.m. collection times ( < 0.05). At the 7:00 a.m., 3:00 a.m., and 5:00 p.m. collection times the difference was close to statistical significance with p-values of $0.09,0.09$ and 0.06 respectively.

A plot of the mean 1-OHPG values in males and females is shown below to illustrate the sex effect at each collection time (Fig.III.24). The p-values associated with the difference between males and females at each collection time are shown on the graph above each time point.

Differences in 1-OHPG levels between males and females with each collection time.

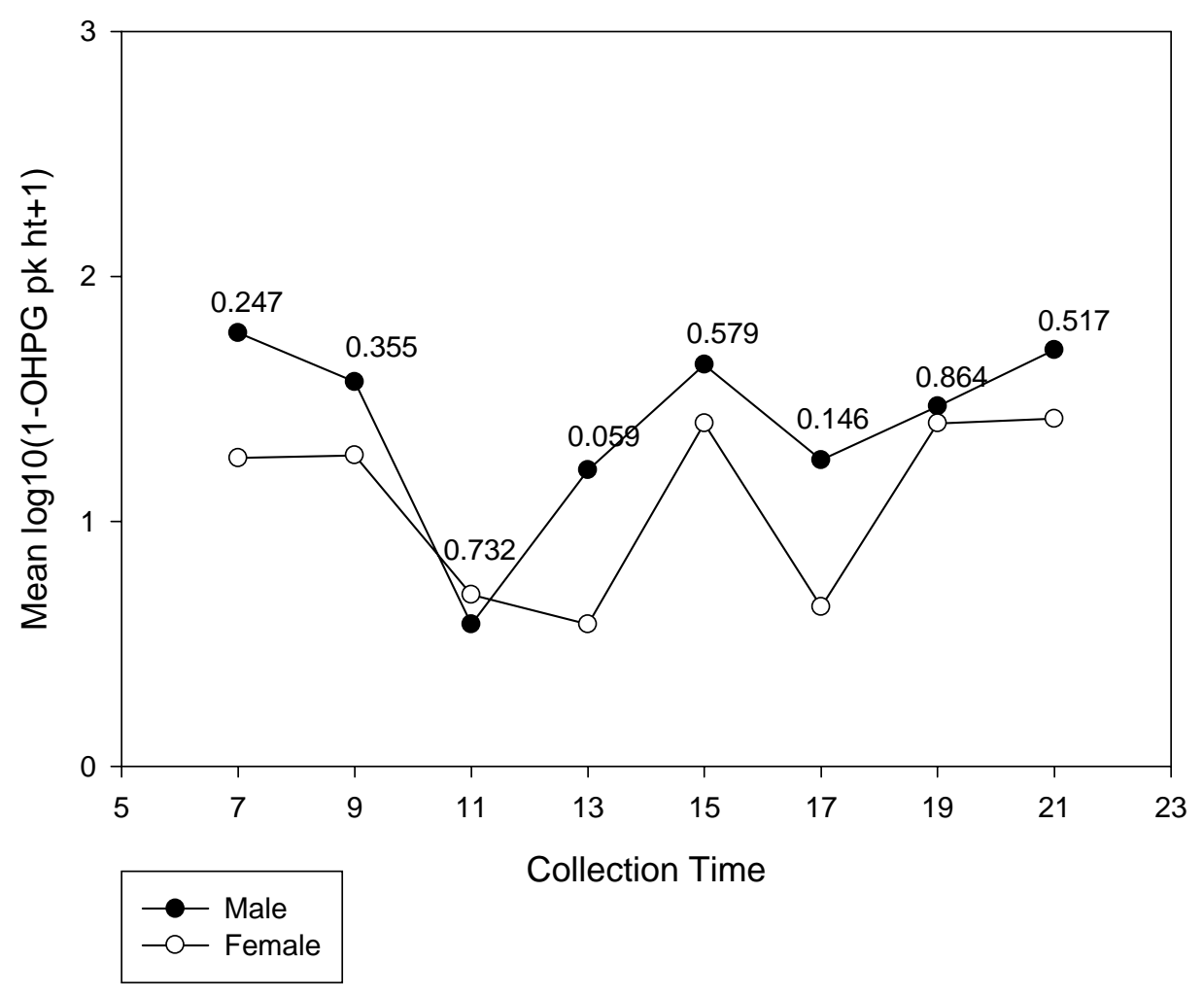

Fig.III.24 Mean 1-OHPG levels males and female with time 
1-OHPG levels between males and females were not significantly different at any of the collection times ( $p$-value $>0.05$ ). To illustrate the smoke*sex*time interaction, plots of mean 1-OHPG levels in male subjects and female subjects is shown P-values associated with differences between smokers and nonsmokers, in each sex category, at each collection times are shown on the figure (Fig.III.25).

1-OHPG levels in male smokers and nonsmoker at different collection times

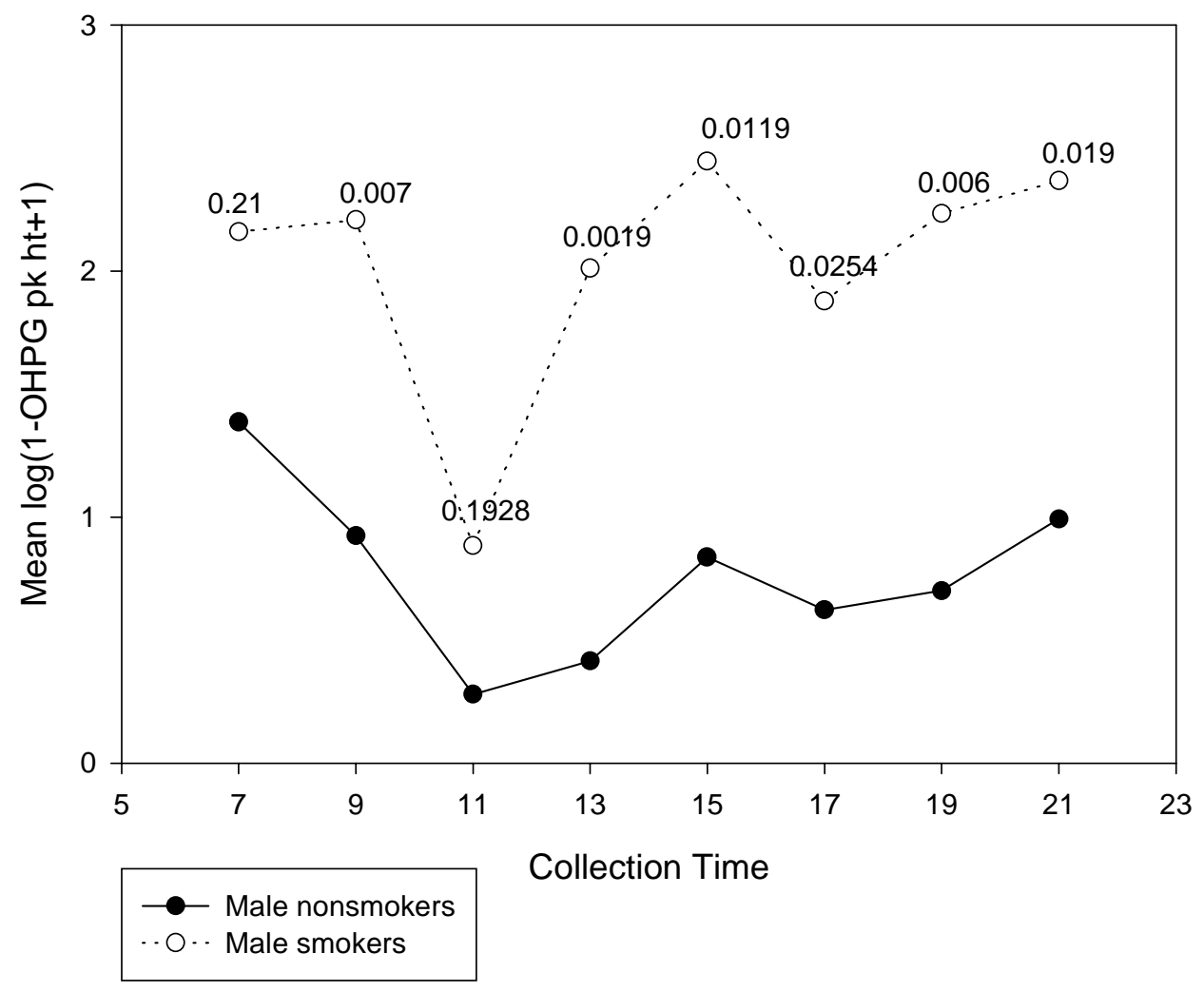

Fig.III.25 Mean 1-OHPG levels males smokers and nonsmokers with time 
1-OHPG levels in male smokers were higher than those in male nonsmokers at all the collection times. The difference reached statistical significance at the 9:00 a.m., 1:00 p.m., 3:00 p.m., 5:00 p.m., 7:00 p.m. and 9:00 p.m. (p<0.05).

1-OHPG levels in female smokers and nonsmoker at different collection times

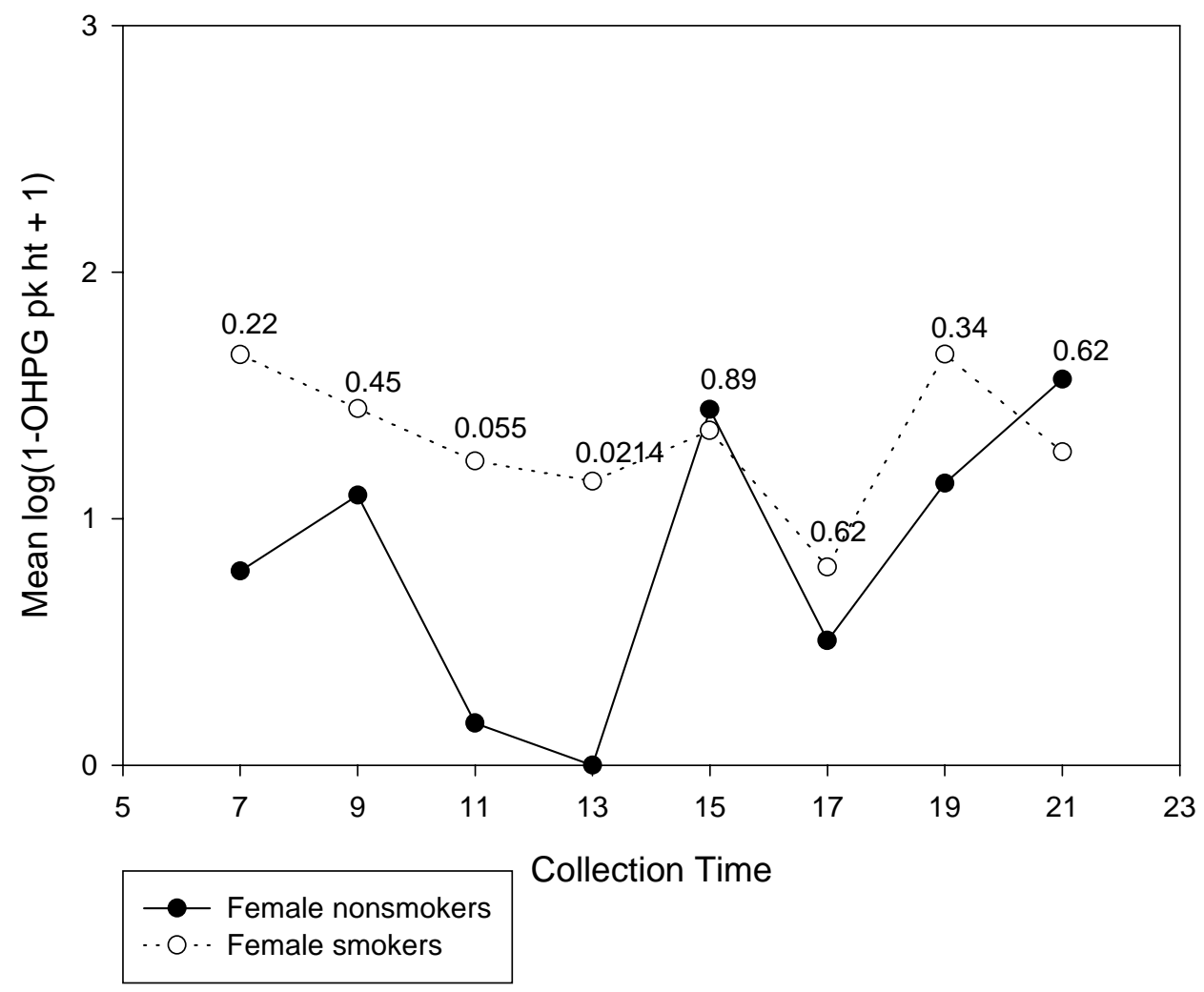

Fig.III.26 Mean 1-OHPG levels females smokers and nonsmokers with time

I-OHPG levels in female smokers were higher than female nonsmokers at the 7:00 a.m., 9:00 a.m., 11:00 a.m., 1:00 p.m., 5:00 p.m., and 7:00 p.m. The difference being statistically significant at the 1:00 p.m $(p=0.02)$ and close to 
significance at the 11:00 a.m. $(\mathrm{p}=0.055)$ collections. Female nonsmoker levels at the 3:00 p.m. and the 9:00 p.m. collections were higher than female smoker levels leading to a significant smoke*sex interaction at the 9:00 p.m. collection $(p=0.049)$ and nearly significant at the 3:00 p.m. collection ( $p=0.06)$. (Fig.III.26).

\section{III.8 Regression of 1-OHPG with the number of cigarettes smoked.}

The dose of pyrene received from cigarette smoke is expected to increase with the number of cigarettes smoked by the person. Thus we expected to see a correlation between the 1-OHPG apparent levels and the number of cigarettes smoked. Log (1-OHPG) values fitted against the number of cigarettes smoked by the individual resulted in a statistically significant linear relationship between the two $(\mathrm{p}=0.0012)$.

The regression equation was:

$\log (1-\mathrm{OHPG}+1)=0.0336($ Number of cigarettes smoked $)+0.812$

The levels of 1-OHPG for nonsmokers was 0.812 as predicted by the above correlation. The correlation coefficient for this equation was 0.646 and the Rsquared was 0.42. (Fig. III.27). 
Correlation between $\log (1-\mathrm{OHPG})$ levels and numbers of cigarettes

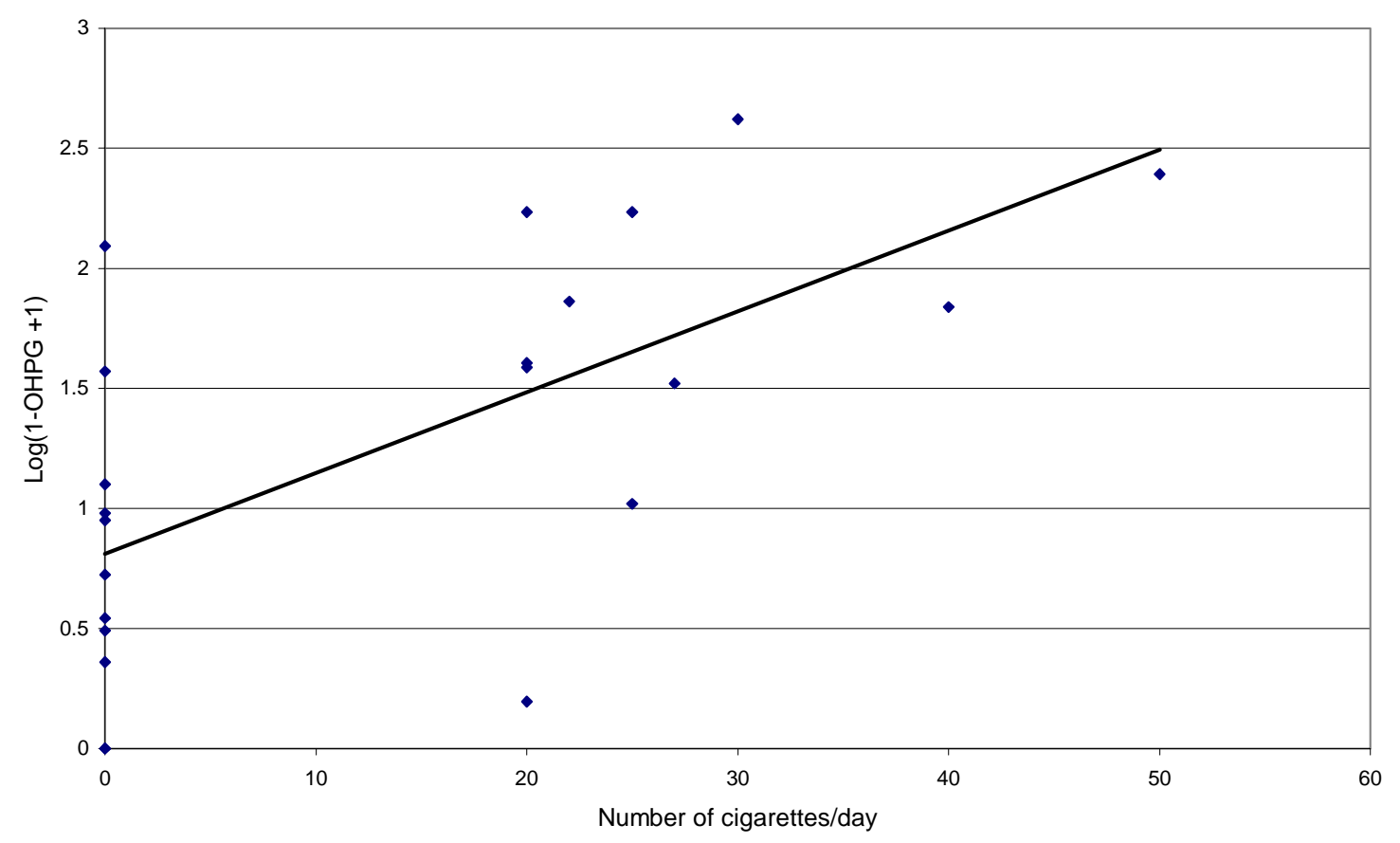

Fig.III.27 Correlation between Log(1-OHPG) levels and number of cigarettes 


\section{CHAPTER-IV}

\section{DISCUSSION}

In the present study increased levels of urinary 1-hydroxypyrene glucuronide were detected in smokers as compared to nonsmokers. Increased urinary levels of 1-hydroxy pyrene have been reported in human biomonitoring studies in subjects exposed to PAH due to occupation, medication, and smoking. (Weston et a.$^{69}$, Merlo et $l l .64$, Gilbert et $l .{ }^{63}$ ). Kang et al. ${ }^{11}$, in a study designed to assess PAH exposure through air to workers at a steel plant, found significantly higher levels of 1-OHPG in smokers compared to nonsmokers. The present study, designed specifically to assess PAH exposure in smokers and nonsmokers not occupationally exposed to PAH and not ingesting high amounts of PAH through diet, reports increased levels of 1-OHPG in smokers $(p=0.0048)$. Merlo et a . $^{64}$ conducted a cross-sectional study among 94 traffic police officers exposed to airborne pollutants and 52 referents exposed to indoor air pollution levels to investigate the relationships between exposure to ambient air PAH and urinary excretion of 1-OHP. They reported 2-fold and 4-fold higher 1-OHP urinary excretions for police officers and referents who smoked $\leq 15$ and $>15$ cigarettes/day respectively in comparison to nonsmokers. Gilbert et al.63 have reported 2-fold higher 1-OHP levels in smokers in comparison to nonsmokers. In this study a 2-fold higher value of log 1-OHPG levels was detected in smokers $(1.71 \pm 0.93)$ as compared to nonsmokers $(0.81 \pm 0.92)$. These results are comparable to those reported for 1-OHP in the Merlo and the Gilbert study. Epidemiological studies have indicated that female tobacco smokers are at higher risk than males of developing lung cancer ${ }^{72,73}$. Higher levels of hydrophobic DNA adduct levels in normal tissue of female as compared to male lung cancer patients supports these epidemiological findings ${ }^{74,75}$. The higher lung cancer risk in female smokers has been attributed to higher susceptibility in females. The Merlo study reported higher urinary excretion of 
1-OHP in female subjects as compared to males. In our study, log 1-OHPG level was about 2.7-fold higher in male smokers $(2.02 \pm 0.78)$ compared to male nonsmokers $(0.77 \pm 0.90)$ and 1.6-fold higher in female smokers (1.33 \pm 0.97$)$ compared to female nonsmokers $(0.84 \pm 0.97)$. Although no statistical significance was seen between male subjects and female, this observation of lower urinary 1-OHPG excretion in female smokers is in contradiction with the Merlo study. Urinary 1-OHPG levels in male smokers observed in our study may have been higher in comparison to those in female smokers due to following possible reasons: a) Male smokers in this study were heavier smokers as compared to female smokers. The average number of cigarettes smoked being $31 \pm 12$ and $22.4 \pm 2.5$ for males and females respectively. b) Smoking habits in males and females were different; all male smokers in this study inhaled deeply in comparison to only 3 of the 5 female smokers. c) Exposure to passive smoking was lower in female subjects; 5 of the 6 male smokers were routinely exposed to passive smoke in comparison to only 2 of the 5 female smokers.

Intake of pyrene through smoking increases proportionally with the number of cigarettes smoked by the individual. It is therefore logical to expect a correlation between pyrene metabolite levels and the number of cigarettes smoked by the individual. Merlo et al. reported a linear relation between the number of cigarettes smoked per day and 1-OHP levels ( $\mathrm{r}^{2}=0.31, \mathrm{P}=0.0001$ ). Correlation between 1-OHP levels and number of cigarettes smoked was also reported by van Rooij el al in a study on male volunteers who were not occupationally exposed to PAHs $\left(\mathrm{r}^{2}=0.67, \mathrm{P}<0.001\right)$. In the present study a positive linear relationship is seen between urinary 1-OHPG levels and number of cigarettes smoked $\left(\mathrm{r}^{2}=0.42, \mathrm{P}<0.0012\right)$. There were only 11 smokers in this study in comparison to 50 in the Merlo study and 76 in the van Rooij study. The correlation between number of cigarettes smoked in the present study probably 
would have been better if there were more number of smokers and a wider range in the number of cigarettes smoked.

Large interindividual differences in the log 1-OHPG values were observed in smokers which was not attributable to the cigarette smoke intake. Intake of pyrene and other PAH from cigarettes varies considerably on individual smoking habits such as depth of inhalation, number of puffs taken per cigarette and the distance from the filter until which the cigarette is smoked. Also the PAH exposure may vary depending on the cigarette brand smoked, tar content, and cigarette length. CYP1A1, involved in the metabolic activation of PAHs, is polymorphically expressed in humans. The capacity of an individual to metabolize procarcinogens can be altered by carrying the variant alleles; genetic polymorphisms of CYP enzymes have been proposed as a biomarker of susceptibility to cancer. Merlo et al. reported that subjects carrying the CYP1A1 MspI polymorphism exhibited higher levels of excretion of 1-OHP if they smoked $\leq 15$ cigarettes/day. Interindividual differences in 1-OHPG metabolism can possibly arise due to metabolic susceptibility gene polymorphisms such as the CYP1A1, and glutathione S-transferase polymorphisms.

Substantial baseline levels of 1-OHPG were observed in nonsmokers in this study, mean 1-OHPG for nonsmokers being $0.8 \pm 0.92$. In the van Rooij study urinary 1-OHP excretion averaged $0.12 \mu \mathrm{mol} / \mathrm{mol}$ creatinine in nonsmokers in comparison to $0.25 \mu \mathrm{mol} / \mathrm{mol}$ creatinine in smokers. Baseline levels of 1-OHP have also been reported in the Merlo study, levels in nonsmokers being approximately half of those in smokers. Our findings of baseline levels in nonsmokers being about one-half, compared to smokers is therefore not unusual. However, in a couple of cases- [FNS\#1 mean log 1-OHPG $=1.57$ and MNS\#5 mean log 1-OHPG $=2.09$ ]- these levels were as high as in smokers categorized by sex (female smokers mean log $1-\mathrm{OHPG}=1.33 \pm 0.96$ and male smokers $=2.02 \pm 0.78$ ). 
Human exposure to pyrene is dependent on a number of sources other than cigarette smoking such as food, environmental tobacco smoke and through occupational exposure. Petrol and diesel engines and industrial emissions release various PAHs into the environment. Increased pyrene exposure due to occupation has been reported among workers in foundries, coking plants, aluminum reduction plants, engine room personnel in ships, petrochemical industries and traffic police officers. None of the subjects in the present study were occupationally exposed to PAHs. Food intake seems to be a major contributor to pyrene levels in nonsmoking humans. Various roasted/baked foods have high pyrene content. Volunteers in our study did not consume foods rich in PAH such as grilled or smoked foods. However, pyrene is present in smaller quantities in a vast variety of foods such as cereals, oils, dairy, sweets, fruits and vegetable. Pyrene dose received from diet can be estimated from the pyrene content of foods, unfortunately the food diaries maintained by volunteers were not detailed enough to allow any quantitative estimation. Van Rooij et al. have reported average dietary pyrene intake of $9.4 \mathrm{nmol} /$ day (range: 3.6 - 23.7 nmol/day). Pyrene intake through mainstream smoke has been reported as $11.91 \mathrm{nmol} /$ day (range: 0.56 - 33.9). Thus, on an average, pyrene exposure in nonsmokers is about one-half of the exposure in smokers. Baseline 1-OHPG levels observed in the present study may be attributable to environmental and dietary sources. Placing subjects on a standardized controlled diet may help eliminating the inter-individual variability in 1-OHPG levels not attributable to cigarette smoke.

A chronobiological effect in the 1-OHPG apparent levels was observed when the mean values were plotted for each collection time. Plot of the mean 1OHPG suggests that levels at the 11:00 a.m. and the 1:00 p.m. collection were relatively low in nonsmokers. In male smokers the levels appeared higher at all the collection times though the differences did not reach statistical significance at the overnight and 11:00 a.m. collection. In female smokers the levels appeared to 
be higher in the samples collected early during the day (overnight, 9:00 a.m., 11:00 a.m., and 1:00 p.m.); the difference being statistically significant only at the 11:00 a.m. and the 1:00 p.m. collections. In the afternoon and evening samples the levels in female nonsmokers and smokers appeared to be roughly equivalent. These findings suggest that morning samples may be better in discerning female smokers from nonsmokers. The highest percentage difference in the 1-OHPG value was found at the 1:00 p.m. collection and the difference was highly significant $(\mathrm{p}=0.0004)$ with mean $1-\mathrm{OHPG}$ value being $1.58 \pm 0.23$ in smokers and $0.21 \pm 0.22$ in nonsmokers. Analysis of data after combining the 11:00 a.m. and 1:00 p.m. values resulted in significantly higher levels in smokers $(p=0.0008)$ with no significant difference between males and females $(p=0.55)$. These findings suggest that analysis of urine sample collected between 9:00 a.m. to 1:00 p.m. (4hour cumulative) may be best to discern between smokers and nonsmokers. Log 1-OHPG value of 1.5 seems to be a convenient cut-off point in discerning between smokers and nonsmokers as most smokers had levels above 1.5 while most nonsmokers had levels below 1.5. More than $50 \%$ of the nonsmoker samples had undetectable levels of $1-\mathrm{OHPG}$ in comparison to only $10 \%$ of the smoker samples.

BaPT levels were detected in only $6 \%$ of the urine samples (10 out of 172 samples) all of which were from smokers (8 male and 2 female). Mean BaPT level in these 10 samples was $230 \mathrm{fmol} / 10 \mathrm{ml}$ urine. BaPT was detected in $80 \%$ of the samples (8 out of 10 samples) ranging from undetectable (below 50 $\mathrm{pmol} / 10 \mathrm{ml}$ ) to $11 \mathrm{pmol} / 10 \mathrm{ml}$ with a mean of $4 \pm 3.4 \mathrm{pmol} / 10 \mathrm{ml}$. Higher levels of $\mathrm{BaPT}$ observed in psoriasis patients is consistent with the fact that exposure to PAH from dermal application of coal tar is substantially higher.

These results are consistent with a study by Weston et al. (50), where levels of $\mathrm{BaPT}$ in psoriasis patients ranged from $<10$ to $330 \mathrm{fmol} / \mathrm{ml}$ and $<10$ to 40 $\mathrm{fmol} / \mathrm{ml}$ in controls. These results suggest that the dose of BaP from cigarette 
smoke or from routine environmental exposure is much lesser than that received from coal tar therapy.

Theoretically, measurement of BaPT could be used as a marker of biological exposure to BPDE-I which is considered as the ultimate carcinogenic metabolite from $\mathrm{BaP}$. The use of urinary $\mathrm{BaPT}$ levels as a biomarker for $\mathrm{PAH}$ exposure can be valid only if its levels can be correlated with existing markers of exposure. Urinary 1-OHP, a metabolite of pyrene estimating the total uptake of PAHs, has been used as a biomarker of individual internal dose. In the present study there was a lack of good correlation between BaPT levels and 1-OHPG levels. However, the number of samples in which BaPT was detected was very less to be conclusive. With the present detection limits of $25 \mathrm{fmol} / \mathrm{ml}$, urinary $\mathrm{BaPT}$ was undetectable in most samples from smokers and does not serve as a biomarker to assess the exposure of an individual to carcinogens from cigarette smoke. More sensitive methods (such as the $\alpha$-cyclodextrin solid matrix-room temperature phosphorescence technique ${ }^{76}$, which has been reported to be 50 times more sensitive for BaPT detection than the SFS technique) may offer better results in future research. BaPT in urine may result from multiple pathways including hydrolysis of BPDE-I, exfoliation of BaP-DNA adducts, glutathione and glucuronide conjugates and protein adducts, further research needs to be done to elucidate the source of $\mathrm{BaPT}$ in urine. Assay sensitivity for BaPT may be compromised due to its epimerization as was seen during methods development. Incidentally, the reversible epimerization of (+/-)-benzo[a]pyrene-r- 7,t-8,9, 10tetrahydro tetrol to (+/-)-benzo[a]pyrene-r-7,t-8,9, c-10-tetrahydro tetrol due to acid hydrolysis has recently been reported by Islam et al.77 They reported an equilibrium composition after acid hydrolysis of $87 \%$ for BaPT which is consistent with our observation of about $85 \%$ BaPT.

1-OHPG is highly fluorescent and may serve as a biomarker for exposure to PAH through cigarette smoke. However, this marker is sensitive to other sources of PAH such as food, environmental smoke and occupational exposure 
and baseline levels are observed in nonsmokers. The use of urinary 1-OHPG concentration as an indicator of total $\mathrm{PAH}$ exposure is valid if the uptake of pyrene is correlated with the uptake of other PAHs, such as the potent carcinogen BaP. Levels of 1-OHP have been shown to correlate well with urinary $3-\mathrm{OH} \mathrm{BaP}(\mathrm{r} 2=0.89)$ in human urine after exposure to $\mathrm{BaP}$ at the workplace. ${ }^{78}$ Experimental demonstration of a correlation between the urinary excretion of 1OHPG and that of other PAH metabolites, such as 3-OH BaP (the major phenol metabolite of $\mathrm{BaP}$ ), would support the validity of 1-OHPG as a biomarker for cigarette carcinogen exposure. Future research may be directed at demonstration of correlation between urinary 1-OHPG levels and urinary 3-OH BaP in smoking volunteers. Also demonstration of correlation between urinary 1-OHPG levels and PAH-DNA adducts in peripheral white blood cells in smokers may be useful in establishing urinary 1-OHPG as a marker of internal dose of carcinogenic PAH from cigarette smoke. 


\section{APPENDIX I}

\section{A-I] Development of extraction method for BaPT using SPE cartridges:}

An extraction method was developed so as to get good extraction efficiency from a solution of BaPT in water using the C-18 Sep-Pak ${ }^{\circledR}$ SPE cartridges. Standard solution having $5 \mathrm{ng} / \mathrm{ml} \mathrm{BaPT}$ in water was prepared by serial dilution of a stock solution as follows: $10 \mu \mathrm{l}$ of a $100 \mu \mathrm{g} / \mathrm{ml} \mathrm{BaPT}$ stock solution was diluted to $1 \mathrm{ml}$ with water to give a $1 \mu \mathrm{g} / \mathrm{ml}$ solution. $10 \mu \mathrm{l}$ of the $1 \mu \mathrm{g} / \mathrm{ml}$ solution thus prepared was diluted to $2 \mathrm{ml}$ with water to give a $5 \mathrm{ng} / \mathrm{ml} \mathrm{BaPT}$ solution.

Extraction procedure used:

Conditioned the Sep-pak ${ }^{\circledR}$ cartridge with $1 \mathrm{ml}$ of methanol followed by $1 \mathrm{ml}$ of water.

Loaded $1 \mathrm{ml}$ of the $5 \mathrm{ng} / \mathrm{ml} \mathrm{BaPT}$ solution onto the column and passed through it with a flow rate of about $1 \mathrm{ml} /$ minute using a Varian sample preparation manifold under vacuum. $2 \mathrm{ml}$ of $30 \%$ methanol in water was run through the column with a flow rate of about $1 \mathrm{ml} / \mathrm{min}$ so as to wash the column. The BaPT held onto the column was then extracted five times using $1 \mathrm{ml}$ of eluant, which was methanol:THF:water $:: 4: 3: 3.25 \mu$ from each extract was injected into a Spheri-5 ODS C-18 Brownlee column, $100 \mathrm{~mm}$ in length and having internal diameter of $4.6 \mathrm{~mm}$. Mobile phase, 50\% methanol in water, was run at a flow rate of $1 \mathrm{ml} /$ minute. Data acquisition was through an online Hitachi ${ }^{\circledR}$ fluorimeter fixed at an excitation wavelength of $349 \mathrm{~nm}$ and an emission wavelength of $379 \mathrm{~nm}$.

Standard BaPT eluted with a retention time of about 7.5 minutes. Peak integration was performed using the Maxima ${ }^{\circledR}$ software and the area under the peak was determined. 
Extraction efficiencies were determined from the area counts of the HPLC peaks as a percent of the standard $5 \mathrm{ng} / \mathrm{ml} \mathrm{BaPT}$ extracted (area count $=218943$ ). Percent extraction from each $1 \mathrm{ml}$ elute is shown in Table A-1. Representative HPLC peak of the extract is shown in Figure A.1

Table A.1 Percent Extraction using Sep-pak

\begin{tabular}{|c|c|c|}
\hline Sample & Area count & $\%$ extraction \\
\hline $1^{\text {st }} \mathrm{ml}$ elute & 0 & 0 \\
\hline $2^{\text {nd }} \mathrm{ml}$ elute & 94535 & $43.18 \%$ \\
\hline $3^{\text {rd }} \mathrm{ml}$ elute & 87254 & $39.85 \%$ \\
\hline $4^{\text {th }} \mathrm{ml}$ elute & 24966 & $11.40 \%$ \\
\hline $5^{\text {th }} \mathrm{ml}$ elute & 4731 & $2.16 \%$ \\
\hline Total & 211486 & $96.59 \%$ \\
\hline
\end{tabular}

BaPT extracted using Sep-pak cartridges

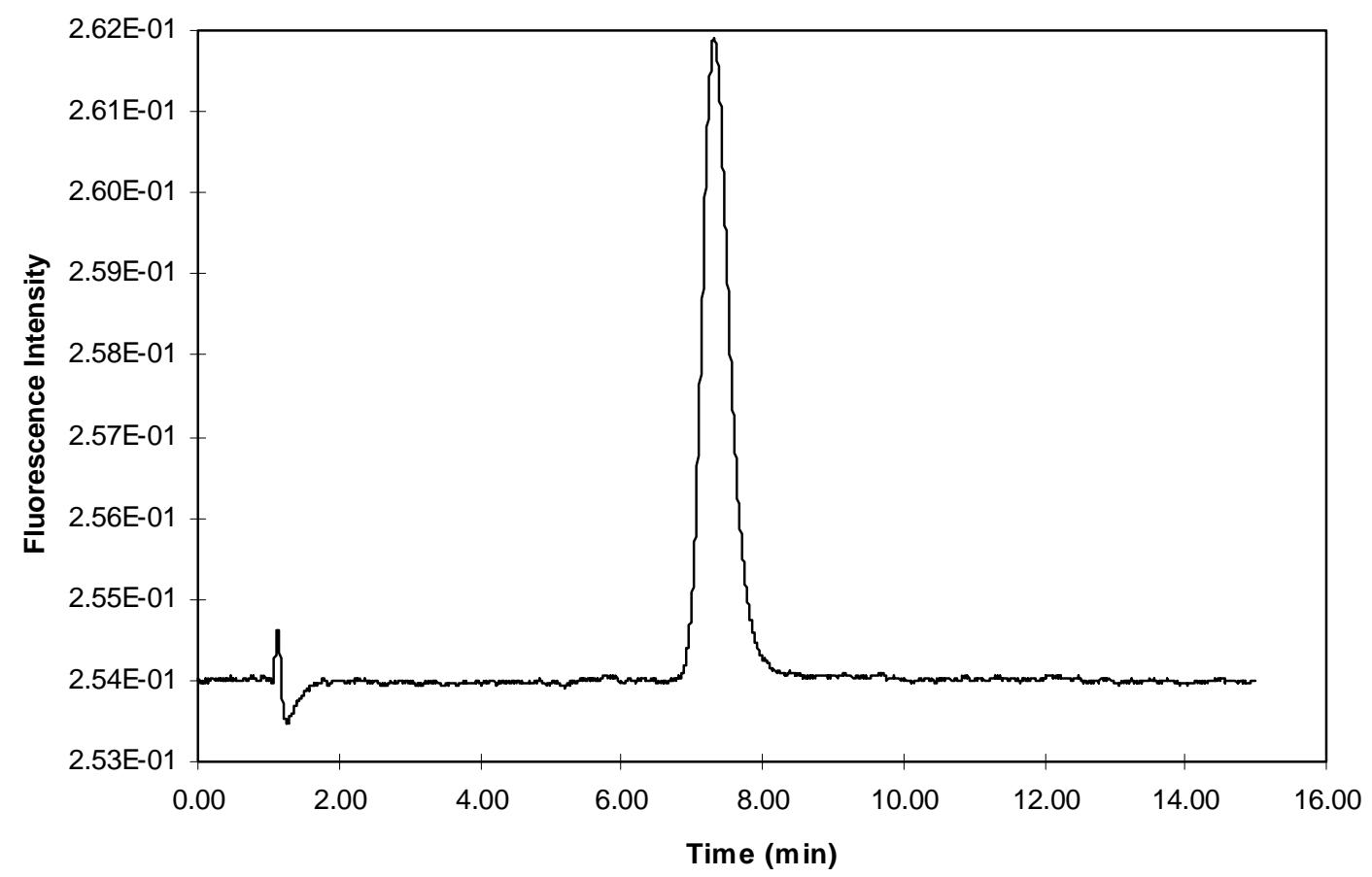

Fig. A.1 Chromatograph of BaPT extracted using Sep-pak cartridges 
The first $1 \mathrm{ml}$ of elute did not extract any BaPT since it only displaced the methanol from the previous wash that had not been removed from the column by vacuum. There was nearly complete extraction using $5 \mathrm{ml}$ of total eluant.

\section{A-II] Extraction of BaPT from urine sample:}

Two nonsmoker urine samples (A and $\mathrm{B}$ ) were spiked with $\mathrm{BaPT}$ to give concentration of $5 \mathrm{ng} / \mathrm{ml}$ of urine. $1 \mathrm{ml}$ of each urine sample was loaded onto the Sep-pak ${ }^{\circledR}$ after conditioning the column with $1 \mathrm{ml}$ of methanol followed by 1 $\mathrm{ml}$ of water. The column was washed as above and extracted with $3 \mathrm{ml}$ of Methanol:THF:water $:: 4: 3: 3$. The experiment was repeated to get 5 readings for each urine sample. HPLC settings were same as above. Area counts were calculated from the chromatographs and are tabulated below in Table A.2

\section{Table A.2 Percent Extraction from spiked urine}

\begin{tabular}{|c|c|c|}
\hline Sample & Area count & \% Extraction \\
\hline Unextracted standard & 289105 & \\
\hline Spiked urine (A)1 & 99520 & $103.27 \%$ \\
\hline Spiked urine (A)2 & 96829 & $100.48 \%$ \\
\hline Spiked urine (A)3 & 95735 & $99.34 \%$ \\
\hline Spiked urine (A)4 & 97451 & $101.12 \%$ \\
\hline Spiked urine (A)5 & 87447 & $90.74 \%$ \\
\hline Spiked urine (B)1 & 101110 & $104.92 \%$ \\
\hline Spiked urine (B)2 & 97100 & $100.76 \%$ \\
\hline Spiked urine (B)3 & 90814 & $94.24 \%$ \\
\hline Spiked urine (B)4 & 91727 & $95.18 \%$ \\
\hline Spiked urine (B)5 & 85267 & $88.48 \%$ \\
\hline
\end{tabular}

The extraction efficiency was $97.85 \pm 3.2$

\section{A-III] Selection of Internal Standard:}

Stereo-isomer of benzo[a]pyrene-r-7,t-8,t-9,c-10-tetrahydro tetrol (analyte), benzo[a]pyrene-r-7,t-8,t-9,t-10-tetrahydro tetrol (IS), was experimented as the 
internal standard. A solution of the analyte and internal standard having a concentration of $100 \mathrm{ng} / \mathrm{ml}$ in water was prepared. $50 \mu \mathrm{l}$ of each of the above solution was diluted to $1 \mathrm{ml}$ to get solutions with concentration of $5 \mathrm{ng} / \mathrm{ml}$ of the analyte (sample A) and internal standard (sample B). Took $25 \mu \mathrm{l}$ from each of the $100 \mathrm{ng} / \mathrm{ml}$ solutions and made up the volume to $1 \mathrm{ml}$ with water (sample C). Injected $25 \mu \mathrm{l}$ of each of the above samples into the HPLC column as described before. The analyte had a retention time of about 7.0 minutes and the internal standard had a retention time of about 15.35 minutes as seen in the chromatographs shown in Figure A.2 and A.3 respectively. The area counts for the peaks were as shown below.(Table A-3)

Table A.3 Selection of internal standard

\begin{tabular}{|c|c|c|}
\hline Sample & Area count & Retention time \\
\hline Sample A & 151848 & $7.008 \mathrm{~min}$ \\
\hline Sample B & 88657 & $15.35 \mathrm{~min}$ \\
\hline Sample C & & \\
\hline $1^{\text {st }}$ peak & 76420 & $7.008 \mathrm{~min}$ \\
\hline $2^{\text {nd }}$ peak & 40947 & $15.35 \mathrm{~min}$ \\
\hline & & \\
\hline
\end{tabular}


Retention time of BaP-7,10/8,9-tetrol

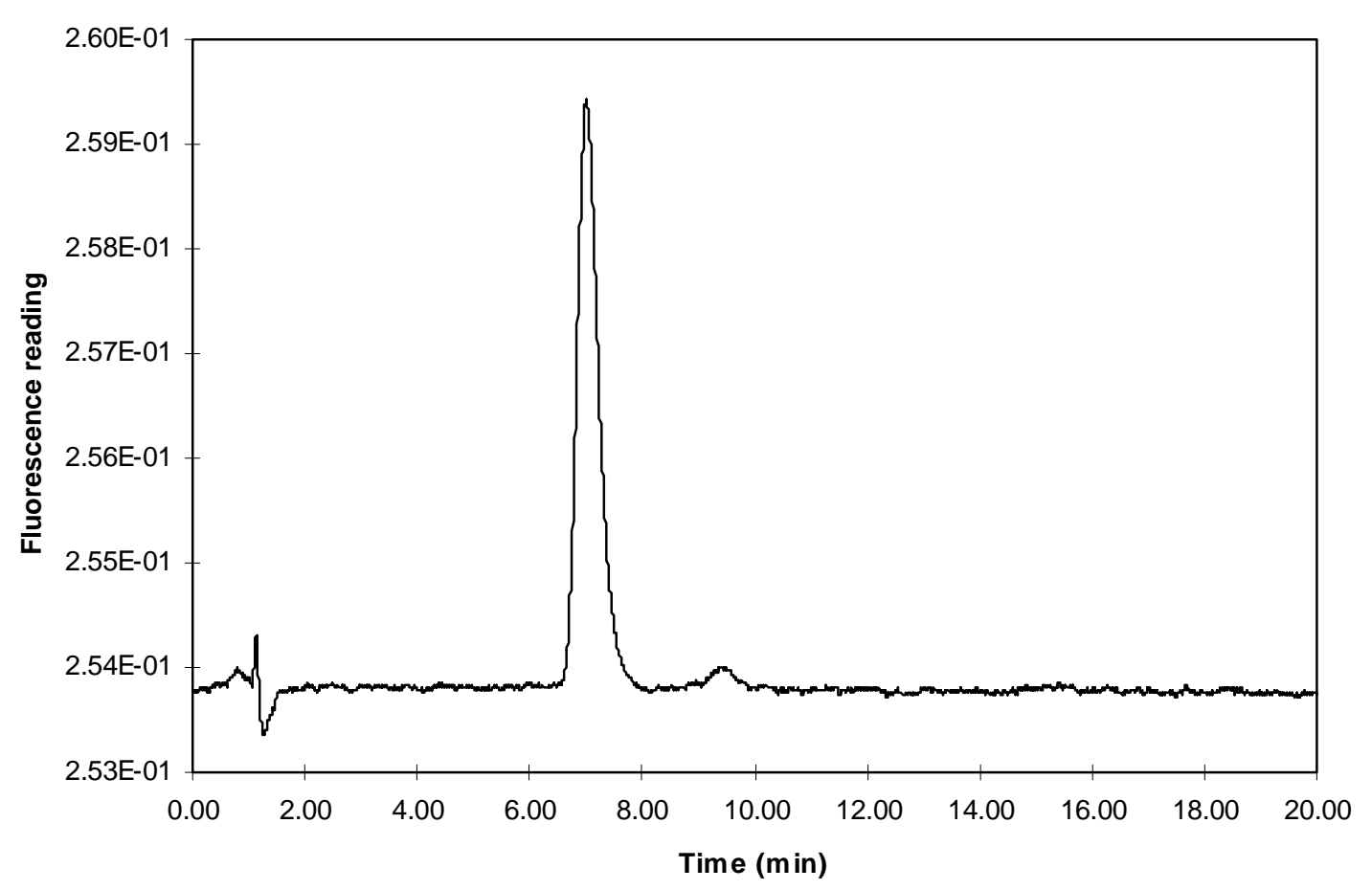

Fig. A.2 Chromatogram of BaP-7,10/8,9-Tetrol 


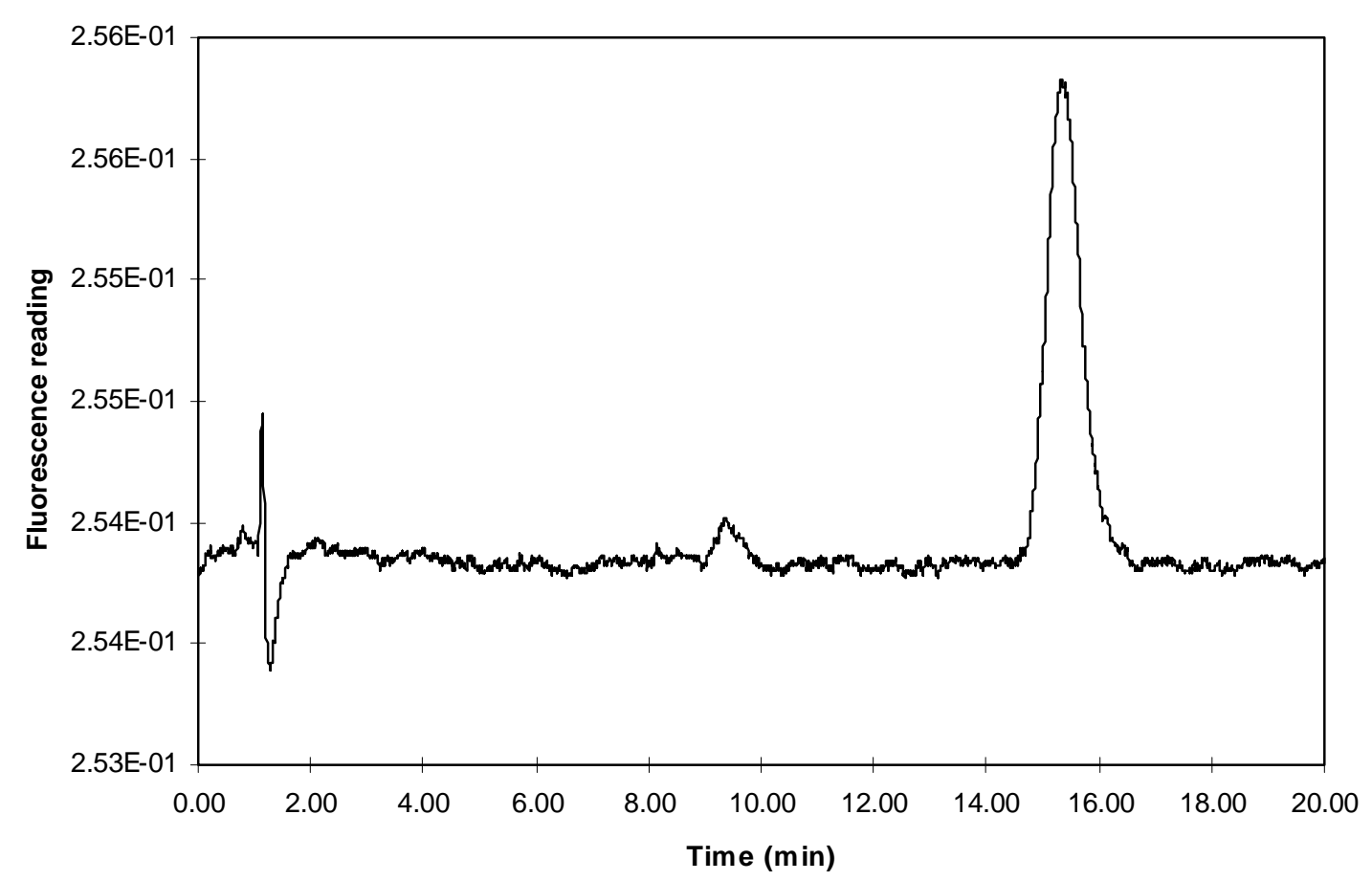

Fig. A.3 Chromatograph of BaP-7/8,9,10-Tetrol

\section{A-IV] Stability of the BaPT and IS:}

Solutions of the analyte and internal standard $(5 \mathrm{ng} / \mathrm{ml}$ each) were prepared in water and urine and extracted using the following procedure:

Condition: $1 \mathrm{ml}$ of methanol followed by $1 \mathrm{ml}$ of water.

Load: $\quad 1 \mathrm{ml}$ of spiked solution

Wash: $\quad 2 \mathrm{ml}$ of $30 \%$ methanol in water

$1 \mathrm{ml}$ of Methanol:THF:water $:: 4: 3: 3$

Extraction: Methanol:THF:water $:: 4: 3: 3$

Injected $25 \mu \mathrm{l}$ of the extract into HPLC column as described before. To assess the degradation and extraction of the samples on exposure to room temperature and light during processing, the same samples as above were kept at room temperature in glass test tubes for a period of 24 hours and then processed 
similarly. The extraction efficiency and the metabolite/IS ratios in the two sets of samples were compared (Table A.4).

Table A.4 A/IS ratio at room temperature

\begin{tabular}{|c|c|c|c|c|c|c|}
\hline Sample & \multicolumn{2}{|c|}{ Analyte } & \multicolumn{2}{c|}{ Internal Standard } & \multicolumn{2}{c|}{ A/IS ratio } \\
\hline & Before & After & Before & After & Before & After \\
\hline Aqueous & 142124 & 147906 & 95356 & 95837 & 1.490457 & 1.543308 \\
\hline Aqueous & 144498 & 141718 & 97175 & 92371 & 1.486987 & 1.534226 \\
\hline Aqueous & 146654 & 145092 & 99149 & 93532 & 1.479127 & 1.551255 \\
\hline Urine 1 & 65853 & 88470 & 50748 & 77799 & 1.297647 & 1.137161 \\
\hline Urine 2 & 63273 & 99357 & 50216 & 77019 & 1.260017 & 1.290032 \\
\hline Urine 3 & 79107 & 86319 & 57507 & 75423 & 1.375606 & 1.144465 \\
\hline
\end{tabular}

The ratios of metabolite/ internal standard were not significantly different before and after exposure to room temperature for 24 hours in both urine and aqueous solutions. The extraction efficiency was low probably due to loss of analyte and IS during extraction or due to quenching of fluorescence in urine. Also the $1 \mathrm{ml}$ of methanol:THF:water :: $4: 3: 3$ in the washing step only displaced the $30 \%$ methanol from the previous wash since it was not completely removed by vacuum. The extraction efficiency was variable from the next $1 \mathrm{ml}$ of methanol:THF:water :: $4: 3: 3$ depending on the extent of $30 \%$ methanol held in the column. To avoid this variability the $30 \%$ methanol should be completely removed from the column by application of vacuum; also washing with $1 \mathrm{ml}$ of methanol:THF:water :: $4: 3: 3$ should not be done. 
Representative chromatograph of sample in Exp. IV after extraction of
BaPT and IS

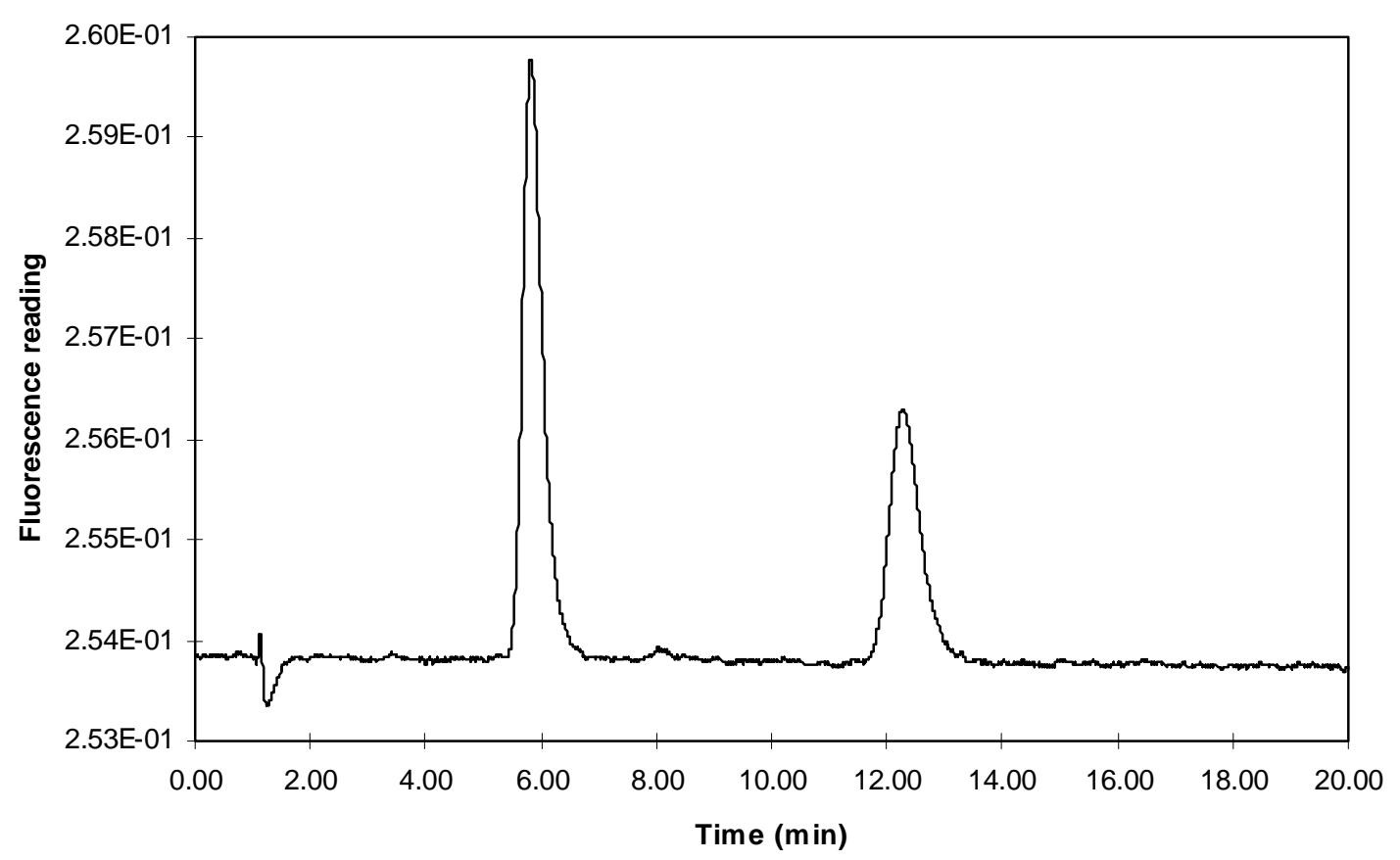

Fig. A.4 Chromatograph of BaPT and IS

\section{A-V] Optimization of the extraction:}

Water spiked with $5 \mathrm{ng} / \mathrm{ml}$ of analyte and internal standard was extracted as follows:

Condition: $1 \mathrm{ml}$ of methanol followed by $1 \mathrm{ml}$ of water.

Load: $\quad 1 \mathrm{ml}$ of the spiked sample.

Wash: $\quad 2 \mathrm{ml}$ of $30 \%$ methanol; let the column run dry completely by vacuum.

Extract: $1 \mathrm{ml}$ of Methanol:THF:water $:: 4: 3: 3$ and run dry each time.

Injected, into HPLC column, $25 \mu \mathrm{l}$ of each extract to determine the percentage of $\mathrm{BaPT}$ extracted in each $1 \mathrm{ml}$ fraction of the extract. The area counts from this experiment are tabulated in the Table A.5. 
Table A.5 Optimization of extraction

\begin{tabular}{|c|c|c|c|}
\hline Sample & Metabolite & IS & Metab/IS \\
\hline Unextracted A & 157916 & 123972 & 1.27 \\
\hline Unextracted B & 143596 & 116050 & 1.24 \\
\hline Unextracted C & 144827 & 115301 & 1.26 \\
\hline \multicolumn{4}{|l|}{ First $1 \mathrm{ml}$ extract } \\
\hline $\mathrm{A}$ & 89333 & 62481 & 1.43 \\
\hline $\mathrm{B}$ & 73455 & 49354 & 1.49 \\
\hline $\bar{C}$ & 81449 & 57283 & 1.42 \\
\hline \multicolumn{4}{|l|}{ Second $1 \mathrm{ml}$} \\
\hline $\mathrm{A}$ & 66126 & 60999 & 1.08 \\
\hline $\mathrm{B}$ & 66563 & 64490 & 1.03 \\
\hline $\mathrm{C}$ & 59135 & 59846 & 0.99 \\
\hline \multicolumn{4}{|l|}{ Third $1 \mathrm{ml}$ extract } \\
\hline $\mathrm{A}$ & 3004 & 4895 & 0.61 \\
\hline B & 2739 & 2402 & 1.14 \\
\hline $\mathrm{C}$ & 1635 & 1563 & 1.05 \\
\hline \multicolumn{4}{|l|}{ Fourth $1 \mathrm{ml}$} \\
\hline $\mathrm{A}$ & 0 & 0 & 0.00 \\
\hline$B$ & 0 & 0 & 0.00 \\
\hline $\mathrm{C}$ & 0 & 0 & 0.00 \\
\hline \multicolumn{4}{|l|}{ Total extraction } \\
\hline $\mathrm{A}$ & 158463 & 128375 & 1.23 \\
\hline $\mathrm{B}$ & 142757 & 116246 & 1.23 \\
\hline $\mathrm{C}$ & 142219 & 118692 & 1.20 \\
\hline
\end{tabular}

The mean extraction efficiency of the analyte in $3 \mathrm{ml}$ of the extract was $99.32 \%$ with a $95 \%$ confidence interval of $98.1-100.54$ and that for the IS was $102.22 \%$ with $95 \%$ confidence interval of 100.18-104.26. The ratio of analyte/IS remained unchanged after extraction. 


\section{A-VI] Analysis of BaPT in urine from smoker and nonsmoker:}

$1 \mathrm{ml}$ urine sample from a smoker (>20 cigarettes/day) and a nonsmoker was processed in the manner described in Experiment $\mathrm{V}$ and extracted with $3 \mathrm{ml}$ of Methanol:THF:water :: 4:3:3. $25 \mu \mathrm{l}$ from this extract was injected into the HPLC column. Same urine samples were spiked with $5 \mathrm{ng} / \mathrm{ml}$ of the analyte and the internal standard each and processed in similar manner along with the urine samples. Area counts from the HPLC peaks are tabulated below in the Table A.6.

Table A.6 A/IS ratio in urine from smoker and nonsmoker

\begin{tabular}{|l|r|r|r|r|r|}
\hline \multicolumn{1}{|c|}{ Sample } & \multicolumn{1}{|c|}{ Analyte } & \multicolumn{1}{c|}{$\%$} & Int.Standar & \multicolumn{1}{c|}{$\%$} & \multicolumn{1}{c|}{ A/IS } \\
\hline Spiked & 121674 & 104.81 & 116949 & 120.11 & 1.04 \\
\hline Spiked & 100062 & 90.32 & 100863 & 101.97 & 0.99 \\
\hline Nonsmoker & 0 & & 0 & & \\
\hline Smoker & 0 & & 0 & & \\
\hline
\end{tabular}

Both smoker and nonsmoker urine did not have detectable levels of BaPT. The levels of BaPT were measurable in the same urine samples spiked with $5 \mathrm{ng} / \mathrm{ml}$ each of the analyte and internal standard. Since the levels of BaPT in the urine samples from both smoker and nonsmoker were below the detection limit, concentration of the extract would be necessary to be able to detect the $\mathrm{BaPT}$ in the urine samples. 
Non-smoker urine spiked with BaPT and IS extracted using Sep-pak cartridges

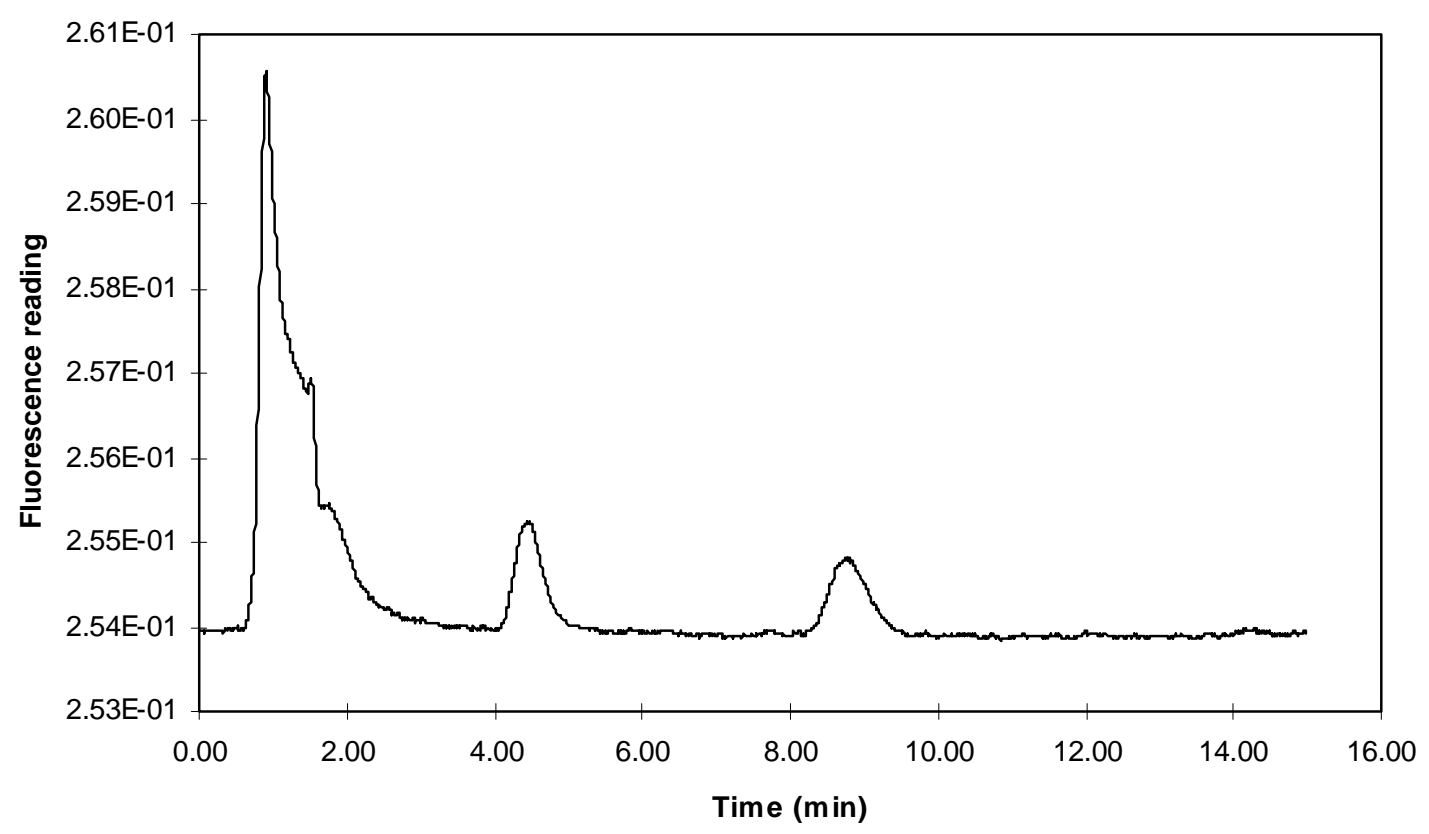

Fig. A.5 Chromatograph of BaPT and IS in spiked urine 
Non-smoker urine extracted using Sep-pak

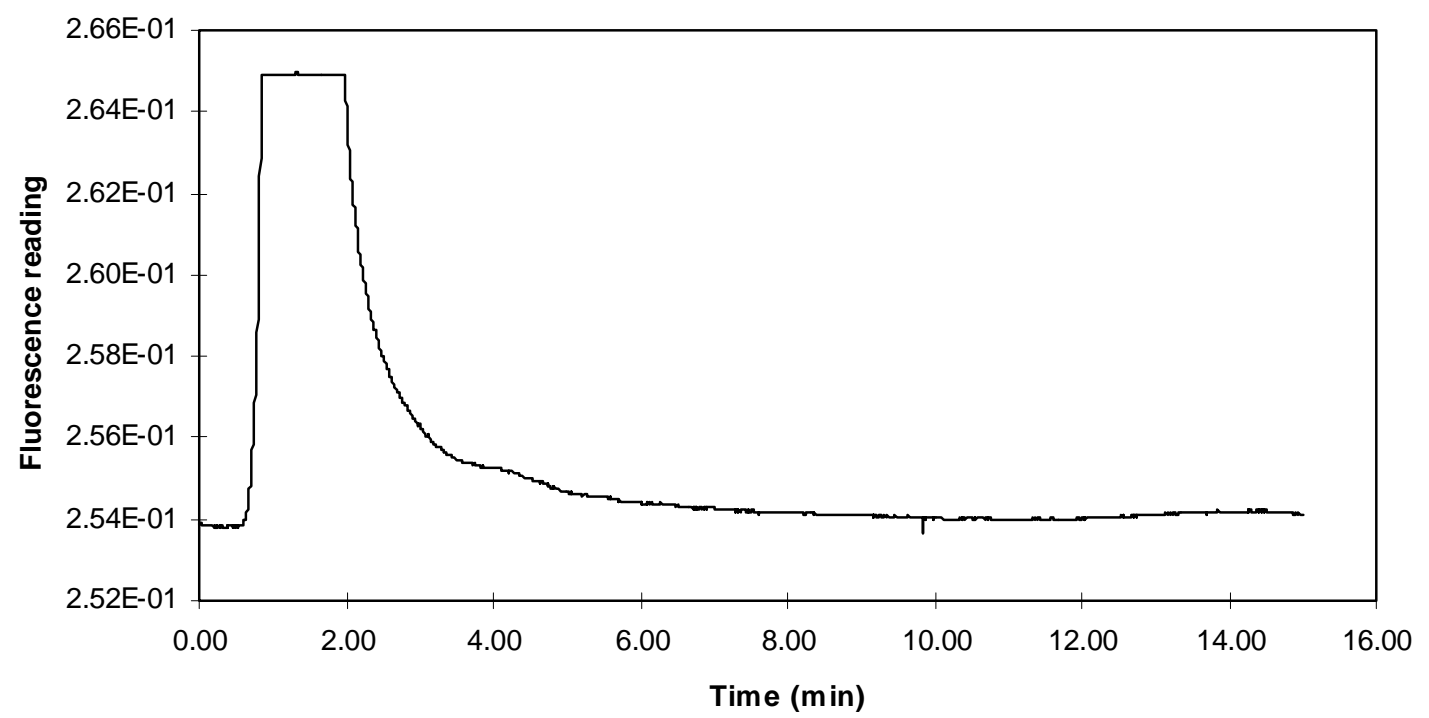

Fig. A.6 Chromatograph of extracted nonsmoker urine

\section{A-VII] Evaporation and reconstitution of extract to increase concentration:}

Extraction of aqueous and urine samples spiked with $5 \mathrm{ng} / \mathrm{ml}$ of analyte and internal standard was done as described previously and the extract was evaporated using a nitrogen evaporator at $40{ }^{\circ} \mathrm{C}$. The residue was then reconstituted in $1 \mathrm{ml}$ of water and then $25 \mu \mathrm{l}$ was injected in HPLC column. Extraction efficiencies were calculated by comparing with the area counts from $25 \mu 1$ of the aqueous samples injected without extraction or concentration. (Table A.7) 
Table A.7 A/IS ratio in urine after concentration

\begin{tabular}{|c|c|c|c|c|c|c|c|c|}
\hline Urine & \multicolumn{3}{|c|}{ Analyte } & \multicolumn{3}{c|}{ Internal standard } & A/IS & A/IS \\
\hline & Extr. & Unext & $\%$ & Extr. & Unext & $\%$ & & \\
\hline A & 12073 & 14212 & 0.8494 & 60261. & 95356 & 0.6319 & 1.34 & 1.49 \\
\hline B & 10196 & 14449 & 0.7056 & 45653. & 97175 & 0.4698 & 1.50 & 1.49 \\
\hline C & 11457 & 14665 & 0.7812 & 47617 & 99149 & 0.4802 & 1.63 & 1.48 \\
\hline Water & & & & & & & & \\
\hline A & 11368 & 15791 & 0.7198 & 83379 & 12397 & 0.6725 & 1.07 & 1.27 \\
\hline B & 10098 & 14359 & 0.7032 & 79432. & 11605 & 0.6844 & 1.03 & 1.24 \\
\hline C & 12058 & 14482 & 0.8326 & 88885 & 11530 & 0.7709 & 1.08 & 1.26 \\
\hline
\end{tabular}

Area counts after evaporation to dryness (nitrogen evaporator) and reconsitution following extraction with $3 \mathrm{ml}$ of Methanol:THF:water :: $4: 3: 3$ gave low extraction efficiency and high variability, this could be due to caking and sticking of the residue to the sides of the tube making it difficult to reconstitute. A rotory vacuum evaporator is more suitable for this step, as the residue stays at the bottom of the tube because of the centrifugal force, making reconstitution easier and less variable. Also extracting greater volume of urine would give higher concentration.

\section{A-VIII] Enzymatic deconjugation of glucuronides:}

A solution of $\beta$-glucuronidase ( Type 4 from Helix aspersa), also having sulfatase activity, was prepared in acetate buffer. Urine samples( smokers and nonsmokers) adjusted to $\mathrm{pH} 5.0$ were incubated at $37^{\circ} \mathrm{C}$, with $10 \mathrm{mg}$ enzyme per $\mathrm{ml}$ of urine, for a period of 24 hours with mild shaking. The same samples were also spiked with $5 \mathrm{ng} / \mathrm{ml}$ of BaPT and incubated with enzyme similarly. After the incubation period the samples were extracted using Sep-pak cartridges as described earlier in experiment V. $25 \mu \mathrm{l}$ of the extract was injected into a Supelcosil ${ }^{\mathrm{TM}} \mathrm{LC}-18$ column having a length of $15 \mathrm{~cm}$ and internal diameter of 4.6 
$\mathrm{mm}$ and a particle size of $5 \mu$. The mobile phase, $50 \%$ methanol was pumped through the column at a flow rate of $1 \mathrm{ml} / \mathrm{min}$. Both smoker and nonsmoker urine had undetectable levels of $\mathrm{BaPT}$ after treatment with the enzymes $\beta$ glucuronidase and sulfatase. BaPT was detectable in the same samples spiked with $5 \mathrm{ng} / \mathrm{ml}$ prior to enzymatic digestion. The extraction of BaPT from urine was quite reproducible but the level of $\mathrm{BaPT}$ present in human urine (both smokers and nonsmokers) was too low to be detected at the limit of detection of 1ng BaPT per ml of urine.

\section{A-IX] Treatment with concentrated hydrochloric acid at $90^{\circ} \mathrm{C}$ :}

$10 \mathrm{ml}$ urine sample was taken in a capped glass tube and $80 \mu \mathrm{l}$ of $38 \%$ hydrochloric acid was added to it. After heating the solution for 3 hours at $90{ }^{\circ} \mathrm{C}$ in a water it was cooled to room temperature and then extracted using Sep-pak cartridges as described before. This acid hydrolysis was done to break down macro-molecular adducts with BaPT and liberate the free tetrol. Both smoker urine and nonsmoker urine had undetectable levels of $\mathrm{BaPT}$ after treatment with concentrated acid to liberate free BaPT from macromolecular adducts. The limit of detection of $1 \mathrm{ng} / \mathrm{ml}$ probably was very high to be able to detect the trace amounts of $\mathrm{BaPT}$ present in human urine. To decrease the limit of detection better sample clean-up was necessary since the Sep-pak cartridges alone did not clean-up the urine well enough for trace analysis. Different types of solid phase extraction cartridges needed to be experimented in order to get better sample clean-up. Also HPLC columns with higher resolving power were to be tried for better resolution from interfering peaks.

\section{A-X] Inter-conversion of BaPT and IS due to heating with $\mathrm{HCl}$ :}

$10 \mathrm{ml}$ nonsmoker urine samples were spiked with $1 \mathrm{ng}$ of benzo[a]pyrene-r-7,t8,t-9,t-10-tetrahydro tetrol (IS) alone; another set of nonsmoker urine samples was spiked with $1 \mathrm{ng}$ of benzo[a]pyrene-r-7,t-8,t-9,c-10-tetrahydro tetrol (BaPT) 
along with $1 \mathrm{ng}$ of IS and subjected to hydrolysis with hydrochloric acid at $90{ }^{\circ} \mathrm{C}$ for 3 hours. The hydrolysed samples were then extracted with Sep pak ${ }^{\mathrm{TM}}$ cartridges, the extract was evaporated to dryness in a Savant SC110 SpeedVac and then reconstituted in $5 \mathrm{ml}$ of Tris- $\mathrm{HCl}$ buffer at $\mathrm{pH}$ of 7.5. The reconstituted solution was loaded onto the immunoaffinity cartridges and extracted as described above. The extract was evaporated to dryness and reconstituted in 100 $\mu \mathrm{l}$ of water and injected into the Prodigy ${ }^{\circledR}$ column. The area counts of the HPLC peaks were calculated and the analyte/IS ratio was determined (shown in Table A.8).

\section{Table A.8 A/IS ratio in urine after heating with $\mathrm{HCl}$}

\begin{tabular}{lccc} 
Sample & AUC & AUC IS & BaPT/IS \\
& BaPT & & \\
A+IS & 180390 & 32565 & 5.5394 \\
A+IS & 247657 & 44009 & 5.6274 \\
A+IS & 231679 & 43327 & 5.3472 \\
A+IS & 215335 & 40782 & 5.2801 \\
A+IS & 217571 & 41796 & 5.2055 \\
A+IS & 221244 & 39600 & 5.5870 \\
A+IS & 234256 & 44281 & 5.2902 \\
Average & 221162 & 40909 & 5.4110 \\
Stdev & 21187 & 4061 & 0.1694 \\
\multicolumn{5}{r}{} & & & \\
Sample & AUC & AUC IS & BaPT/IS \\
& BaPT & & \\
IS & 153356 & 27400 & 5.5969 \\
IS & 200601 & 35310 & 5.6811 \\
IS & 205736 & 38719 & 5.3136 \\
IS & 209185 & 39947 & 5.2366 \\
Average & 192220 & 35344 & 5.4571 \\
Stdev & 26148 & 5648 & 0.2152
\end{tabular}

The ratio of analyte : Internal standard was about 5.46 in both the set of samples indicating that the IS was undergoing an epimerization reaction on heating with 
$\mathrm{HCl}$ to the BaPT. Percentages of analyte: IS at equilibrium after heating is 85:15. Thus, we decided not to use of benzo[a]pyrene-r-7,t-8,t-9,t-10-tetrahydro tetrol as the internal standard.

\section{A-XI] Extraction cartridges:}

The $3 \mathrm{ml}$ Supelclean LC-SCX cartridge containing sulfonic acid bonded silica as solid phase (strong cation exchange) and $3 \mathrm{ml}$ Supelclean LC-SAX cartridge containing quaternary amine bonded silica (strong anion exchange) were tried to get better sample clean-up. Solid phase extraction by ion exchange is based on the attraction between a charged analyte and an oppositely charged packing in the SPE tube. Although BaPT is non-ionic, urine contains many ionic compounds which would be retained onto the ion exchange cartridges and the elute from these cartridges would contain the non-ionic metabolites in a relatively cleaner matrix. Urine spiked with $2.5 \mathrm{ng}$ of BaPT and IS was passed through both the SCX and SAX cartridges after conditioning with $1 \mathrm{ml}$ of methanol followed by 1 $\mathrm{ml}$ of water. The elute from both the columns was evaporated to dryness using a Savant SC110 SpeedVac, reconstituted in water and extracted using the C-18 Seppak columns as before. The phenyl boronic acid (PBA) column from Varian Sample Preparation Products was experimented for extraction of BaPT from urine. The immobilized phenyl boronic acid was equilibrated with $5 \mathrm{ml}$ of $3 \%$ phosphoric acid in methanol and ammonium sulfate at $\mathrm{pH} 8.5$ to form the reactive boronate form $\mathrm{RB}(\mathrm{OH})_{3-}-2.5 \mathrm{ng}$ of the $\mathrm{BaPT}$ solution in acetate buffer at $\mathrm{pH} 5.0$ was loaded onto the column. Washings were given with $2 \mathrm{ml}$ of water, 1 $\mathrm{ml}$ of methanol, and $1 \mathrm{ml}$ of $50 \%$ acetonitrile in $0.01 \mathrm{M}$ ammonium sulfate at $\mathrm{pH}$ 8.5. Elution was done with $2 \mathrm{ml}$ of $0.5 \%$ phosphoric acid.

The Waters Oasis HLB, Waters Sep-pak, Supelco Envichrom P, Supelco LC-18, Supelco LC-CN, and Supelco LC-Ph were also experimented for extraction efficiency and sample clean-up using the following procedure: 
Conditioning of the column was done with $1 \mathrm{ml}$ of methanol followed by $1 \mathrm{ml}$ of water, the sample was then loaded onto the column at a flow rate of about 1 $\mathrm{ml} / \mathrm{min}$. The column was then washed with $6 \mathrm{ml}$ of water, followed by $2 \mathrm{ml}$ of $30 \%$ methanol in water. Elution was done with $3 \mathrm{ml}$ of $80 \%$ methanol in water. The extract was then injected into a Supelcosil ${ }^{\mathrm{TM}} \mathrm{ABZ}+$ column with mobile phase, $50 \%$ methanol, running at a flow rate of $0.1 \mathrm{ml} / \mathrm{min}$. Percentage extraction was calculated from the areas under the HPLC peaks using Maxima software.

Sample clean-up after passing through the SCX, SAX and the C-18 Sep-pak was similar to that obtained by the C-18 Sep-pak alone. Phenyl boronic acid columns have been used for the extraction of various vicinal diols, 1,3-diols and 1,3,5triols but the retention of the $\mathrm{BaP}$ tetrol on the PBA columns was not satisfactory probably due to stearic hinderence caused by the bulky structure of BaPT. The extraction efficiencies of the various columns tried are shown in Table A.9

\section{Table A.9 Extraction efficiency of various columns}

\begin{tabular}{|c|c|}
\hline Column & Extraction \\
\hline Waters C-18 Sep-pak & $86 \%$ \\
\hline Waters Oasis HLB & $82 \%$ \\
\hline Supelco LC-18 & $82 \%$ \\
\hline Supelco LC-PH & $78 \%$ \\
\hline Supelco Envirochrom P & $77 \%$ \\
\hline
\end{tabular}

Sample clean-up from all the above columns was similar. In order to get better sample clean-up to be able to measure trace levels of BaPT a more selective separation technique such as immunoaffinity column chromatography would be necessary. 


\section{APPENDIX - II}

The following table gives the urine collection data from the volunteers and the peak heights obtained by SFS spectra and the calculated value based on the urine volume collected.

Value $=$ UrineVolume $*$ Peak height $/$ Volume of urine used for analysis $(\mathrm{ml})$

$$
\begin{aligned}
& \text { MS }=\text { male smoker } \quad \text { FS }=\text { female smoker } \\
& \text { MNS = male nonsmoker } \quad \text { FNS }=\text { female nonsmoker } \\
& \text { N.A. = Not available }
\end{aligned}
$$

$\begin{array}{lllll}\text { Name } & \text { CollectionTime } & \text { Peak ht } & \text { UrineVolume } & \text { Value } \\ & & & & \\ \text { MS1 } & \text { Ovn } & 15 & 205 & 307.5 \\ \text { MS1 } & 9 \mathrm{a} & 41.86 & 120 & 502.32 \\ \text { MS1 } & 11 \mathrm{a} & 9.15 & 82 & 75.03 \\ \text { MS1 } & 1 \mathrm{p} & 40.41 & 158 & 319.239 \\ \text { MS1 } & 3 \mathrm{p} & 8.93 & 100 & 89.3 \\ \text { MS1 } & 5 \mathrm{p} & 4.13 & 66 & 27.258 \\ \text { MS1 } & 7 \mathrm{p} & 23.63 & 175 & 413.525 \\ \text { MS1 } & 9 \mathrm{p} & 15.5 & 120 & 186 \\ \text { MS2 } & \text { Ovn } & 2.69 & 1205 & 324.145 \\ \text { MS2 } & 9 \mathrm{a} & 6.6 & 145 & 95.7 \\ \text { MS2 } & 11 \mathrm{a} & 0 & 112 & 0 \\ \text { MS2 } & 1 \mathrm{p} & 12 & 87.3 & 104.76 \\ \text { MS2 } & 3 \mathrm{p} & 37 & 95.2 & 352.24 \\ \text { MS2 } & 5 \mathrm{p} & 6.61 & 165 & 109.065 \\ \text { MS2 } & 7 \mathrm{p} & 6.54 & 141 & 92.214 \\ \text { MS2 } & 9 \mathrm{p} & 2.55 & 164 & 41.82 \\ \text { MS3 } & \text { Ovn } & 16.52 & 325 & 536.9 \\ \text { MS3 } & 9 \mathrm{a} & 25.87 & 101 & 261.287 \\ \text { MS3 } & 11 \mathrm{a} & 19.68 & 14.75 & 29.028 \\ \text { MS3 } & 1 \mathrm{P} & \mathrm{N} . \mathrm{A} . & \text { No collection } & \text { N.A. } \\ \text { MS3 } & 3 \mathrm{p} & 248.29 & 54.2 & 1345.7318 \\ \text { MS3 } & 5 \mathrm{p} & 22.84 & 100 & 228.4 \\ \text { MS3 } & 7 \mathrm{p} & 114.25 & 77.45 & 884.86625 \\ \text { MS3 } & 9 \mathrm{p} & 199.88 & 95.6 & 1910.8528\end{array}$




\begin{tabular}{|c|c|c|c|c|}
\hline MS4 & Ovn & 6.8 & 175 & 119 \\
\hline MS4 & $9 a$ & 11.2 & 100 & 112 \\
\hline MS4 & $11 \mathrm{a}$ & 0 & 110 & 0 \\
\hline MS4 & $1 \mathrm{p}$ & 7.42 & 48 & 35.616 \\
\hline MS4 & $3 p$ & 13.16 & 88 & 115.808 \\
\hline MS4 & $5 p$ & 2.33 & 82 & 19.106 \\
\hline MS4 & $7 \mathrm{p}$ & 4.36 & 56 & 24.416 \\
\hline MS4 & $9 \mathrm{p}$ & 4.05 & 120 & 48.6 \\
\hline MS5 & Ovn & 0 & 1000 & 0 \\
\hline MS5 & $9 a$ & 7 & 191 & 133.7 \\
\hline MS5 & $11 a$ & 0 & 223 & 0 \\
\hline MS5 & $1 \mathrm{p}$ & 5.8 & 199 & 115.42 \\
\hline MS5 & $3 p$ & 5.71 & 228 & 130.188 \\
\hline MS5 & $5 p$ & 3.65 & 220 & 80.3 \\
\hline MS5 & $7 \mathrm{p}$ & 17.23 & 62.7 & 108.0321 \\
\hline MS5 & $9 \mathrm{p}$ & 28 & 136.5 & 382.2 \\
\hline MS6 & Ovn & 25.41 & 542 & 1377.222 \\
\hline MS6 & $9 a$ & 12 & 73.7 & 88.44 \\
\hline MS6 & $11 \mathrm{a}$ & 10.02 & 87.5 & 87.675 \\
\hline MS6 & $1 \mathrm{p}$ & 7.03 & 110 & 77.33 \\
\hline MS6 & $3 p$ & 58.17 & 124 & 721.308 \\
\hline MS6 & $5 p$ & 14.97 & 104.5 & 156.4365 \\
\hline MS6 & $7 \mathrm{p}$ & 30.84 & 85.5 & 263.682 \\
\hline MS6 & $9 \mathrm{p}$ & 58.15 & 93.25 & 542.24875 \\
\hline FS1 & Ovn & N.A. & No collection & N.A. \\
\hline FS1 & $9 a$ & 0 & 56 & 0 \\
\hline FS1 & $11 \mathrm{a}$ & 32.8 & 25.9 & 84.952 \\
\hline FS1 & $1 p$ & 0 & 23 & 0 \\
\hline FS1 & $3 p$ & 7.58 & 94.6 & 71.7068 \\
\hline FS1 & $5 p$ & N.A. & No collection & N.A. \\
\hline FS1 & $7 \mathrm{p}$ & 9.64 & 215 & 207.26 \\
\hline FS1 & $9 p$ & 0 & 105 & 0 \\
\hline FS2 & Ovn & 2.57 & 450 & 115.65 \\
\hline FS2 & $9 a$ & 1.44 & 200 & 28.8 \\
\hline FS2 & $11 a$ & 0.78 & 175 & 13.65 \\
\hline FS2 & $1 \mathrm{p}$ & 1.5 & 190 & 28.5 \\
\hline FS2 & $3 p$ & 0.46 & 195 & 8.97 \\
\hline FS2 & $5 p$ & 3.34 & 170 & 56.78 \\
\hline FS2 & $7 \mathrm{p}$ & 1.96 & 160 & 31.36 \\
\hline FS2 & $9 p$ & 12 & 150 & 180 \\
\hline FS3 & ovn & 0 & 475 & 0 \\
\hline FS3 & $9 a$ & 6.38 & 57 & 36.366 \\
\hline FS3 & $11 \mathrm{a}$ & 0 & 155 & 0 \\
\hline
\end{tabular}




$\begin{array}{lllll}\text { FS3 } & 1 \mathrm{p} & 0 & 220 & 0 \\ \text { FS3 } & 3 \mathrm{p} & 0 & 135 & 0 \\ \text { FS3 } & 5 \mathrm{p} & 0 & 50 & 0 \\ \text { FS3 } & 7 \mathrm{p} & 0 & 165 & 0 \\ \text { FS3 } & 9 \mathrm{p} & 0 & 145 & 0 \\ \text { FS4 } & \text { ovn } & 4.33 & 400 & 173.2 \\ \text { FS4 } & 9 \mathrm{a} & 1.43 & 120 & 17.16 \\ \text { FS4 } & 11 \mathrm{a} & \text { N.A. } & \text { No collection } & \text { N.A. } \\ \text { FS4 } & 1 \mathrm{p} & 53.58 & 59 & 316.122 \\ \text { FS4 } & 3 \mathrm{p} & 2.11 & 101 & 21.311 \\ \text { FS4 } & 5 \mathrm{p} & 3.61 & 76 & 27.436 \\ \text { FS4 } & 7 \mathrm{p} & 28.73 & 67 & 192.491 \\ \text { FS4 } & 9 \mathrm{p} & 27.3 & 63 & 85.995 \\ \text { FS5 } & \text { ovn } & 6.96 & 320 & 222.72 \\ \text { FS5 } & 9 \mathrm{a} & 51.79 & 162 & 838.998 \\ \text { FS5 } & 11 \mathrm{a} & 18.61 & 36 & 66.996 \\ \text { FS5 } & 1 \mathrm{p} & 8.2 & 74 & 60.68 \\ \text { FS5 } & 3 \mathrm{p} & 39.25 & 95.5 & 374.8375 \\ \text { FS5 } & 5 \mathrm{p} & 0 & 120 & 0 \\ \text { FS5 } & 7 \mathrm{p} & 12.06 & 135 & 162.81 \\ \text { FS5 } & 9 \mathrm{p} & 3.65 & 385 & 140.525\end{array}$

$\begin{array}{lllll}\text { MNS1 } & \text { ovn } & 0 & 190 & 0 \\ \text { MNS1 } & 9 \mathrm{a} & 0 & 20 & 0 \\ \text { MNS1 } & 11 \mathrm{a} & 0 & 65 & 0 \\ \text { MNS1 } & 1 \mathrm{p} & 0 & 160 & 0 \\ \text { MNS1 } & 3 \mathrm{p} & 0 & 45 & 0 \\ \text { MNS1 } & 5 \mathrm{p} & 0 & 70 & 0 \\ \text { MNS1 } & 7 \mathrm{p} & 0 & 100 & 0 \\ \text { MNS1 } & 9 \mathrm{p} & 0 & 130 & 0 \\ \text { MNS2 } & \text { ovn } & 5.25 & 270 & 141.75 \\ \text { MNS2 } & 9 \mathrm{a} & 19.21 & 35.75 & 68.67575 \\ \text { MNS2 } & 11 \mathrm{a} & 5.87 & 80 & 46.96 \\ \text { MNS2 } & 1 \mathrm{p} & 5.04 & 218 & 109.872 \\ \text { MNS2 } & 3 \mathrm{p} & 28.33 & 522 & 1478.826 \\ \text { MNS2 } & 5 \mathrm{p} & 10.95 & 132.4 & 144.978 \\ \text { MNS2 } & 7 \mathrm{p} & 8.82 & 45.5 & 40.131 \\ \text { MNS2 } & 9 \mathrm{p} & 14.98 & 77.1 & 115.4958 \\ \text { MNS3 } & \text { ovn } & 3.07 & 61 & 18.727 \\ \text { MNS3 } & 9 \mathrm{a} & 7.51 & 17 & 12.767 \\ \text { MNS3 } & 11 \mathrm{a} & 0 & 175 & 0\end{array}$




\begin{tabular}{|c|c|c|c|c|}
\hline MNS3 & $1 p$ & 0.33 & 54 & 1.782 \\
\hline MNS3 & $3 p$ & 7.6 & 91 & 69.16 \\
\hline MNS3 & $5 p$ & 4.43 & 82 & 36.326 \\
\hline MNS3 & $7 p$ & 3.21 & 60 & 19.26 \\
\hline MNS3 & $9 p$ & 0 & 170 & 0 \\
\hline MNS4 & ovn & 2.71 & 695 & 188.345 \\
\hline MNS4 & $9 a$ & 4.02 & 22 & 8.844 \\
\hline MNS4 & $11 \mathrm{a}$ & 0 & 112 & 0 \\
\hline MNS4 & $1 p$ & 0 & 46 & 0 \\
\hline MNS4 & $3 p$ & 0 & 76 & 0 \\
\hline MNS4 & $5 p$ & 0 & 133 & 0 \\
\hline MNS4 & $7 p$ & 1.75 & 105 & 18.375 \\
\hline MNS4 & $9 p$ & 1.13 & 140 & 15.82 \\
\hline MNS5 & ovn & 0.94 & 265 & 24.91 \\
\hline MNS5 & $9 a$ & 0 & 122 & 0 \\
\hline MNS5 & $11 a$ & 0 & 150 & 0 \\
\hline MNS5 & $1 p$ & 0 & 184 & 0 \\
\hline MNS5 & $3 p$ & 0 & 160 & 0 \\
\hline MNS5 & $5 p$ & 0 & 151 & 0 \\
\hline MNS5 & $7 p$ & 0 & 122 & 0 \\
\hline MNS5 & $9 p$ & 4.89 & 58 & 28.362 \\
\hline MNS6 & ovn & 0.87 & 160 & 13.92 \\
\hline MNS6 & $9 a$ & 4.2 & 85 & 35.7 \\
\hline MNS6 & $11 a$ & 0 & 75 & 0 \\
\hline MNS6 & $1 p$ & 0 & 90 & 0 \\
\hline MNS6 & $3 p$ & 0 & 67 & 0 \\
\hline MNS6 & $5 p$ & 0 & 93 & 0 \\
\hline MNS6 & $7 p$ & 0 & 106 & 0 \\
\hline MNS6 & $9 p$ & 2.66 & 55 & 14.63 \\
\hline FNS1 & ovn & 0 & 83 & 0 \\
\hline FNS1 & $9 a$ & 16.02 & 16 & 25.632 \\
\hline FNS1 & $11 \mathrm{a}$ & 0.8 & 75 & 6 \\
\hline FNS1 & $1 p$ & 0 & 117 & 0 \\
\hline FNS1 & $3 p$ & 23.62 & 180 & 425.16 \\
\hline FNS1 & $5 p$ & 15.29 & 210 & 321.09 \\
\hline FNS1 & $7 p$ & 21.17 & 65 & 137.605 \\
\hline FNS1 & $9 p$ & 114.98 & 82 & 942.836 \\
\hline FNS2 & ovn & 2.42 & 215 & 52.03 \\
\hline FNS2 & $9 \mathrm{a}$ & 4.06 & 37.9 & 15.3874 \\
\hline FNS2 & $11 \mathrm{a}$ & 0 & 158 & 0 \\
\hline FNS2 & $1 p$ & 0 & 101 & 0 \\
\hline FNS2 & $3 p$ & 2.28 & 189 & 43.092 \\
\hline FNS2 & $5 p$ & 0 & 140 & 0 \\
\hline
\end{tabular}




$\begin{array}{lllll}\text { FNS2 } & 7 \mathrm{p} & 5.73 & 92 & 52.716 \\ \text { FNS2 } & 9 \mathrm{p} & 2.39 & 130 & 31.07 \\ \text { FNS3 } & \text { ovn } & 8.05 & 200 & 161 \\ \text { FNS3 } & 9 \mathrm{a} & 3.14 & 39.9 & 12.5286 \\ \text { FNS3 } & 11 \mathrm{a} & 0 & 138 & 0 \\ \text { FNS3 } & 1 \mathrm{p} & 0 & 149 & 0 \\ \text { FNS3 } & 3 \mathrm{p} & 3.81 & 172 & 65.532 \\ \text { FNS3 } & 5 \mathrm{p} & 0 & 197 & 0 \\ \text { FNS3 } & 7 \mathrm{p} & 5.76 & 119 & 68.544 \\ \text { FNS3 } & 9 \mathrm{p} & 6.13 & 104 & 63.752 \\ \text { FNS4 } & \text { ovn } & 0 & 317 & 0 \\ \text { FNS4 } & 9 \mathrm{a} & 0 & 194 & 0 \\ \text { FNS4 } & 11 \mathrm{a} & 0 & 56.1 & 0 \\ \text { FNS4 } & 1 \mathrm{p} & 0 & 46.2 & 0 \\ \text { FNS4 } & 3 \mathrm{p} & 0 & 82 & 0 \\ \text { FNS4 } & 5 \mathrm{p} & 0 & 65.8 & 0 \\ \text { FNS4 } & 7 \mathrm{p} & 0 & 56.8 & 0 \\ \text { FNS4 } & 9 \mathrm{p} & 0 & 45 & 0 \\ \text { FNS5 } & \text { ovn } & 0 & 280 & 0 \\ \text { FNS5 } & 9 \mathrm{a} & 4.19 & 118 & 49.442 \\ \text { FNS5 } & 11 \mathrm{a} & 0 & 74.3 & 0 \\ \text { FNS5 } & 1 \mathrm{p} & 0 & 110.5 & 0 \\ \text { FNS5 } & 3 \mathrm{p} & 1.69 & 71.7 & 12.1173 \\ \text { FNS5 } & 5 \mathrm{p} & 0 & 66.2 & 0 \\ \text { FNS5 } & 7 \mathrm{p} & 0 & 85.8 & 0 \\ \text { FNS5 } & 9 \mathrm{p} & 7.08 & 46.9 & 33.2052\end{array}$




\section{BIBLIOGRAPHY}

1. U.S Department of Health and Human Services. Nurses: Help Your Patients Stop Smoking. DHHS publication 90-2962. Washington DC: National Heart, Lung, and Blood Institute. 1990

2. Fisher EB, Bishop DB, Goldm untz J, Jacobs A. Implications for the practicing physician of the psychosocial dimension of smoking: Chest, 93:69S, 1988

3. Cancer facts and Figures 1999. American Cancer Society, Atlanta, GA.

4. U.S. Environmental Protection Agency (USEPA). Respir atory Health Effects of Passive Smoking: Lung Cancer and Other Disorders. EPA/600/6-90/006F. Washington DC: Office of Research and Development. 1992

5. Doll R, Peto $\mathrm{T}$ et al. Cigarette smoking and bronchial carcinoma dose and time relationships among regular smokers and lifelong nonsmokers: J. Epidemiol Commun Health, 32(4), p. 303-313, 1978.

6. U.S. Department of Health and Human Services: The Health Consequences of Smoking: Cardiovascular Disease. A Report of the Surgeon General Department of Health and Human Services, Public Health Service, Office on Smoking and Health, DDHS Publ. No. (PHS) 84-50204, p.369, 1983.

7. Ibid; p.370.

8. Horn D.: Who is Quitting ---And Why. In, Schwartz, J.L(Ed.): Progress in Smoking Cessation. Proceedings of the International Conference on Smoking Cessation, American Cancer Society, New York : p.31,June 1978.

9. Engstrom PF, Rosvold EA, Boyd NR Jr., and Orleans TC. Prevention of Tobacco-Related Cancers.--- In Holland JF et al. Cancer Medicine, Fourth edition, Volume I, p.459

10. Schwartz JL. Review and Evaluation of Smoking Cessation Methods: The United States and Canada 1978-1985. U.S. Department of Health and Human 
Services, Public Health Service, National Institutes of Health. NIH Publication No. 87-2940. 1987

11. Fiore M, Novontny T, Pierce J, et al. Methods used to quit smoking in the United States: do cessation programs help? : JAMA 268: p.2687-2694. 1990

12. Ockene JK, Kuller LH, Svendsen KH, Meilahn E. The relationship of smoking cessation to coronary heart disease and lung cancer in the multiple risk factor intervention trial, (MRFIT). Am Public Health, 80(8): p.954-958, 1978.

13. Millatmal T, Daughton D et al: Smoking reduction : An alternative approach for smokers who cannot quit. Monaldi Arch Chest Dis, 49(5): p.421-424, 1994.

14. Hammond EC. Smoking in relation to the death rates of one million men and women. In: Epidemiological Approaches to the Study of Cancer and Other Chronic Diseases. NCI Monograph 19, Jan 1966, p.127 - 204.

15. Hartge P, Siverman D, Hoover R, Schairer C, Altman R, Austin D, Myers MH, Narayana A, Sullivan JW, Swanson GM, Thomas D, West D. Changing cigarette habits and bladder cancer risk: A case - control study. Journal of the National Cancer Institute, 78(6): p.1119 - 1125, June 1987.

16. Kahn HA. The Dorn study of smoking and mortality among U.S. veterans: report on eight and one half years of observation. In: Epidemiological Approaches to the Study of Cancer and Other Chronic Diseases. NCI Monograph 19, Jan 1966, p.1 - 125

17. Slaughter DP, Southwick HW, Smejkal W. "Field cancerization" in oral stratified squamous epithelium: Clinical implications of multicentric origin. Cancer, 85:1453, 1953

18. Silverman S.Jr, Greenspan D, Gorsky M. Tobacco usage in patients with head and neck carcinomas: a follow-up study on habit changes and second 
primary oral/oropharyngeal cancers. Journal of American Dental Association, 106(1): p.33 - 35, 1983.

19. Chemistry and Analysis of Tobacco Smoke in IARC Monographs on the Evaluation of the Carcinogenic Risk of Chemicals to Humans, World Health Organization, International Agency for Research on Cancer,38: 83 - 126, 1985.

20. Hoffmann D, Hecht SS. Advances in tobacco carcinogenesis. Handb.Exp.Pharmacol., 94/I,63, 1990.

21. Kappas A, Alvares AP, Anderson KE, Pantuck EJ, Pantuck CB, Chang R and Conney, AH. Effect of charcoal-broiled beef on antipyrine and theophylline metabolism. Clin. Pharmacol. Ther., 23(4): p.445-450, 1978.

22. Buckley TJ, Waldman JM, Dhara R, Greenberg A, Ouyang Z, Lioy PJ. An assessment of a urinary biomarker for total human environmental exposure to benzo[a]pyrene. Int. Arch. Occup. Envron. Health, 67: p.257-266, 1995.

23. Melikian AA, Bagheri K, Hecht SS, Hoffmann D. Metabolism of benzo[a]pyrene and 7 beta, 8 alpha-dihydroxy-9-alpha, 10 alpha-epoxy7,8,9,10-tetrahydrobenzo[a]pyrene in lung and overt of newborn mice. Chemico-Biological interactions, 69(2-3): p.245 - 57, 1989.

24. MacLeod MC, Adair G, Dickson-Black D, Pevny T, Humphrey RM. Stabilization of a reactive, electrophilic carcinogen, benzo[a]pyrene diol epoxide, by mammalian cells: Chemico- Biological Interactions, 63(3): p.279$289,1987$.

25. Van Rooij JC, Veeger MM, Bodelier-Bade MM; Scheepers PT, Jongeneelen FJ. Smoking and dietary intake of polycyclic aromatic hydrocarbons as sources of interindividual variability in the baseline excretion of 1-hydroxypyrene in urine. Int. Arch.Occup. Environ. Health, 66(1): p.55-65, 1994.

26. Rous P, and Kidd JG. Conditional neoplasms and subthreshold neoplastic states. J. Exp. Mes. 74:365, 1941. 
27. Berenblum I. The cocarcinogenic action of croton resin. Cancer Research 1:44, 1941

28. Berenblum I. The mechanism of carcinogenesis: A study of the significance of cocarcinogenic action and related phenomena. Cancer Research 1:807, 1941

29. Berenblum I. The effect of podophyllotoxin on the skin of the mouse, with reference to carcinogenic, cocarcinogenic, and anticarcinogenic action. Journal of the National Cancer Institute; p.839-841,1951.

30. Nesnow S, Triplett LL and Slaga TJ. Mouse skin tumor initiation-promotion and complete carcinogenesis bioassays: mechanisms and biological activities of emission samples. Environmental Health Perspectives. Vol. 47, p. 255-268, 1983.

31. Slaga TJ, Sivak, A, and Boutwell RK. Carcinogenesis--a comprehensive survey; Volume-2. Mechanisms of tumor promotion and cocarcinogenesis. Raven Press, New York, 1978.

32. Barrett JC and Wiseman RW. Cellular and molecular mechanisms of multistep carcinogenesis: relevance to carcinogen risk assessment. Environ. Health Perspect. 7: p.65-70. 1987.

33. Yuspa SH, and Poirier MC. Chemical carcinogenesis: from animal models to molecular models in one decade. Adv. Cancer Res; 50: p.25-70,1988.

34. Poirier MC, Fullerton NF, Smith BA and Beland FA. DNA adduct formation and tumorigenesis in mice during the chronic administration of 4aminobiphenyl at multiple dose levels. Carcinogenesis, 16(12): p.2917-2921, 1995.

35. Barrett JC. Mechanisms of multistep carcinogenesis and carcinogen risk assessment. Environmental Health Perspectives. Vol. 100, p 9-20, 1993

36. Poirier MC, Beland FA. Aromatic amine DNA adduct formation in chronically-exposed mice: considerations for human comparison. Mutation Research, 376 (1-2): p.177-184, 1997. 
37. Swanson DH, Walling JF. Use of ultrasonics in the rapid extraction of Hi-Vol filters for benzo[a]pyrene (BaP) analysis. Chromatography Newsletter, 9: 25 $-6,1981$.

38. Fazio T, White RH, Howard JW. Collaborative study of the multicomponent method for polycyclic aromatic hydrocarbons in foods. J Assoc Offic Anal Chem, 56(1): p.68-70, 1973.

39. The FTC Cigarette Test Method for Determining Tar, Nicotine, and Carbon monoxide yields of U.S. Cigaretttes. Report of the NCI Expert Committee: Smoking and Tobacco Control, Monograph 7, National Institutes of Health, National Cancer Institute, 1996.

40. Buckley JB, Waldman JM, Dhara R, Greenberg A, Ouyang Z, Lioy PJ. An assessment of a urinary biomarker for total human environmental exposure to benzo[a]pyrene Int Arch Occup Environ Health, 67(4): p.257-266, 1995.

41. Federal Register (May 29, 1992) Guidelines for exposure assessment. 57(104): 22887-22938.

42. Hoffmann D and Brunnemann KD. Endogenous formation of Nnitrosoproline in cigarette smokers. Cancer Res, 43(11): p.5570-5574, 1983.

43. Clonfero E, Jongeneelen FJ, Zordan M and Levis AG. Biological monitoring of human exposure to coal tar. Urinary mutagenesis assay and analytical determination of PAH-metabolites in urine. In: Complex Mixtures and Cancer Risk. Eds. Vainio, H; Sorsa M and McMichael A.J. IARC Sci Publ Lyon (France), 104: p.215-222, 1990.

44. Poirier MC; Weston A; Gupta-Burt S; Reed E. Measurement of DNA adducts by immunoassays. Basic Life Sci; 53:1-11. 1990

45. Reddy MV, and Randerath K. 32P-postlabelling assay for carcinoge-DNA adducts: nclease PI-mediated enhancement of its sensitivity and applications. Environ.Health Perspect; 76: p.41-47, 1987. 
46. Shuker DE, Prevost MD, Friesen D, Lin H, Ohshima and Bartsch H. Urinary markers for measuring exposure to endogenous and exogenous alkylating agents and precursors. Environ. Health Perspect.,99: p.33-37, 1993.

47. Weston A, Bowman D, Manchester DK and Harris CC. Fluorescence detection of lesions in DNA. Basic Life Sciences, 53: 63-81, 1990.

48. Floyd RA, Watson JJ, Wong PK, Altmiller DH and Rickard RC. Hydroxyl free radical adduct of deoxyguanosine: sensitive detection and mechanisms of formation. Free Radical Res. Commun, 1(3): p.163-172, 1986.

49. Weston A. Physical methods for the detection of carcinogen-DNA adducts in humans. Mutation Research, 288(1): p.19-29, 1993.

50. Chacko M, Gupta RC. Evaluation of DNA damage in the oral mucosa of tobacco users and non-users by 32P-adduct assay. Carcinogenesis, 9(12): p.2309-2313, 1988.

51. Shields PG, Bowman ED, Harrington AM, Doan VT and Weston A. Polycyclic Aromatic Hydrocarbon-DNA Adducts in Human Lung and Cancer Susceptibility Genes. Cancer Research, 53(15): p.3486-3492, 1993.

52. Phillips DH, Ni She M. Smoking-related DNA adducts in human cervical biopsies. IARC Sci Publ., 124: p.327-30, 1993.

53. Santella RM. Application of new techniques for the detection of carcinogen adducts in human population monitoring. Mutation Research, 205(1-4): p.271-282, 1988.

54. Becher G, BjØrseth A. Determination of Exposure To Polycyclic Aromatic Hydrocarbons By Analysis of Human Urine. Cancer Letters, 17(3): p.301-311, 1983.

55. Ariese F, Verkaik M, Hoornweg GP, van de Nesse RJ, Jukema-Leenstra SR, Hofstraat JW, Gooijer C, Velthorst NH. Trace Analysis of 3-Hydroxy Benzo[a]pyrene in Urine for the Biomonitoring of Human Exposure to 
Polycyclic Aromatic Hydrocarbons. Journal of Analytical Toxicology, 18(4): p.195-204,1994.

56. Jongeneelen FJ, Anzion RBM,Leijdekkers, Ch.M,Bos RP and Henderson P.Th. 1-Hydroxypyrene in human urine after exposure to coal tar and a coal tar derived product. Int. Arch. Occup. Environ. Health, 57(1): p.47-55, 1985.

57. Jongeneelen GJ, Anzion RBM, Henderson PT. Determination of hydroxylated metabolites of polycyclic aromatic hydrocarbons in urine. J. Chromatography, 413: p.227-232, 1987.

58. Jongeneelen FJ, Akker W. van de and Bos RP. 1-Hydroxypyrene as an indicator of the mutagenicity of coal tar after activation with human liver preparations. Mutation Research, 204(2): p.203-206, 1988.

59. Clonfero E, Zordan $\mathrm{M}$ and Venier P. Biological monitoring of human exposure to coal tar. Urinary excretion of total polycyclic aromatic hydrocarbons, 1-hydroxypyrene and mutagens in psoriatic patients. Int. Arch. Occup. Environ.Health, 61(6): p.363-368, 1989.

60. Roggi C, Minoia C, Sciarra GF, Apostoli P, Maccarini L, Magnaghi S, Cenni A, Fonte A, Nidasio GG, Micoli G. Urinary 1-hydroxypyrene as a marker of exposure to pyrene: an epidemiological survey on a general population group. Sci. Total Environ., 199(3): p.247-254, 1997.

61. Boogaard PJ, Sittert van NJ. Urinary 1-hydroxypyrene as biomarker of exposure to polycyclic aromatic hydrocarbons in workers in petrochemical industries: Baseline values and dermal uptake. Sci. Total Environ.,163(1-3): p.203-209, 1995.

62. Moen BE, Nilsson R, Nordlinder R, ØvrebØ S, Bleie K, Skorve AH, Hollund BE. Assessment of exposure to polycyclic aromatic hydrocarbons in engine rooms by measurement of urinary 1-hydroxypyrene. Occup Environ Med., 53(10): p.692-696, 1996. 
63. Gilbert N, Viau C. Biological monitoring of environmental exposure to PAHs in the vicinity of a Soderberg aluminium reduction plant. Occup Environ Med., 54(8): p.619-621, 1997.

64. Merlo F, Andreassen A, Weston A, Pan CF, Haugen A, Valerio F, Reggiardo G, Fontana V, Garte S, Puntoni R and Abbondandolo A. Urinary Excretion of 1-Hydroxypyrene as a Marker for Exposure to Urban Air Levels of Polycyclic Aromatic Hydrocarbons. Cancer Epidemiology, Biomarkers \& Prevention, 7(2): p.147-155, 1998.

65. Strickland PT, Kang D, Bowman ED, Fitzwilliam A, Downing TE, Rothman N, Groopman JD and Weston A. Identification of 1-hydroxypyrene glucuronide as a major pyrene metabolite in human urine by sychronous fluorescence spectroscopy and gas chromatography - mass spectrometry. Carcinogenesis, 15(3): p.483-487, 1994.

66. Kang DH, Rothman N, Poirier MC, Greenberg A, Hsu CH, Schwartz BS, Baser ME, Groopman JD, Weston A and Strickland PT. Interindividual differences in the concentration of 1-hydroxypyrene-glucuronide in urine and polycyclic aromatic hydrocarbon-DNA adducts in peripheral white blood cells after charbroiled beef consumption. Carcinogenesis, 16(5): p.1079-1085, 1995.

67. Uziel M, Ward RJ, Vo-Dinh T. Synchronous Fluorescence Measurement of BaP Metabolites in Human and Animal Urine. Analytical Letters, 20(5): p.761-776, 1987.

68. Weston A, Bowman ED, Carr P, Roghman N and Strickland PT. Detection of metabolites of polycyclic aromatic hydrocarbons in human urine. Carcinogenesis, 14(5): p.1053-1055, 1993.

69. Weston A, Santella RM and Bowman ED. Detection of Polycyclic Aromatic Hydrocarbon Metabolites in Urine From Coal Tar Treated Psoriasis Patients and Controls. Polycyclic Aromatic Compunds, 5: p.241-247, 1994. 
70. Santella RM, Lin CD, Cleveland WL and Weinstein IB. Monoclonal antibodies to DNA modified by a benzo[a]pyrene diol epoxide. Carcinogenesis, 5(3): p.373-377, 1984.

71. Kang D, Rothman N, Cho SH, Lim HS, Kwon HJ, Kin SM, Schwartz B, Strickland PT. Association of exposure to polycyclic aromatic hydrocarbons (estimated from job category) with concentration of 1-hydroxypyrene glucuronide in urine from workers at a steel plant. Occupational and Environmental Medicine, Vol.52, No.9, p.593-599, 1995.

72. Risch HA, Howe GR, Jain M, Burch JD, Holowaty EJ, Miller AB. Are female smokers at higher risk for lung cancer than male smokers? A case-control analysis by histologic type. Am J epidemiol., Sep 1;138(5): p.281-93, 1993.

73. Zang EA, Wynder EL. Differences in lung cancer risk between men and women: examination of the evidence. J Natl Cancer Inst., Feb 21, 88(3-4): 183-92, 1996.

74. Kure EH, Ryberg D, Hewer A, Phillips DH, Skaug V, Baera R, Haugen A. p53 mutations in lung tumours: relationship to gender and lung DNA adduct levels: Carcinogenesis, Oct;17(10): p.2201-5, 1996.

75. Ryberg D, Hewer A, Phillips DH, Haugen A. Different susceptibility to smoking-induced DNA damage among male and female lung cancer patients. Cancer Research, Nov 15,54:p.5801-5803, 1994.

76. Johannes Corley, Robert J.Hurtubise, Elise Bowman and Ainsley Weston. Solid matrix, room temperature phosphorescence identification and quantitation of the tetrahydrotetrols derived from the acid hyfrolysis of benzo[a]pyrene-DNA adducts from human lung. Carcinogenesis vol. 16 no.2 p.423-426, 1995.

77. Islam GA, Greibrokk T, Rise F, Ovrebo S. Epimerization of benzo[a]pyrenetetrols after acid hydrolysis, implications for determination of 
benzo[a]pyrene adducts in protein and DNA. Mutatation Research 423(1-2) p.47-54, Jan 251999

78. Freek Ariese; Marjeke Verkaik; Gerard P.Hoornweg; Ronald J.van de Nesse; Swanette R; Jukema-leenstra; Johannes W.Hofstraat; Cees Gooijer, and Nel H. Velthorst. Trace analysis of 3-hydroxy benzo[a]pyrene in urine for the biomonitoring of human exposure to polycyclic aromatic hydrocarbons: Journal of Analytical Toxicology, 18: July.August 1994. 